\title{
Estudos em Otimização Linear
}

\author{
Ricardo Silveira Sousa
}

Orientador: Prof. Dr. Marcos Nereu Arenales

Dissertação apresentada ao Instituto de Ciências Matemáticas e de Computação - ICMC-USP, como parte dos requisitos para obtenção do titulo de Mestre em Ciências - Área: Ciências de Computação e Matemática Computacional.

USP - São Carlos

Junho de 2000 
Aos meus queridos pais,

João Geraldo de Souza e Maria de Lourdes Silveira, em gratidão por tamanho e contínuo apoio no conquistar de novos desafios...

E a minha filha Yara Gomes Sousa. 


\section{Agradecimentos}

Mencionar todas as pessoas que me apoiaram na realização desta dissertação seria um trabalho impossível, todavia, desejo expressar minha sincera e profunda gratidão agradecendo a:

- A minha noiva Maria Luiza pelo grande amor, carinho e incentivo durante todo o desenvolvimento deste trabalho;

- Ao professor Marcos Nereu Arenales pela amizade, incentivo permanente e por suas valiosas orientações que me conduziram na realização deste trabalho;

- Ao meu irmão Rodrigo e ao grande amigo Toninho, que me apoiaram a todo momento;

- Aos meus amigos e colegas de trabalho: Fernando, Bibi, Gilmar, Zé, Randal, Belluzzo, Marcelo, Silvio, Thiago, Wellington, Araxá, Lilian, Kattiana, Heloisa, Luiz Fernando, Cris Nogueira, Maristela, Gisele, Maju, Fabão e ao Sandro Montezano;

- Professores e funcionários do ICMC - USP pelo convívio diário, amizade e respeito;

- A FAPESP e ao CNPQ pelo apoio financeiro;

- Principalmente a Deus, por está ao meu lado a todo instante. 


\section{Sumário}

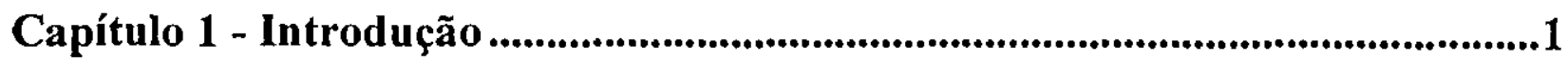

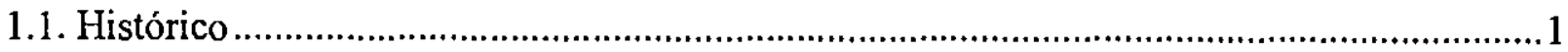

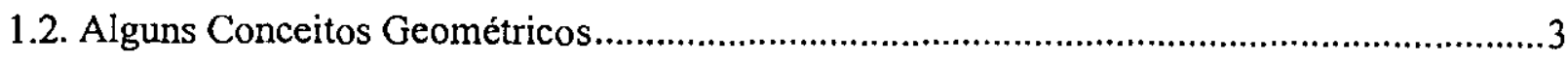

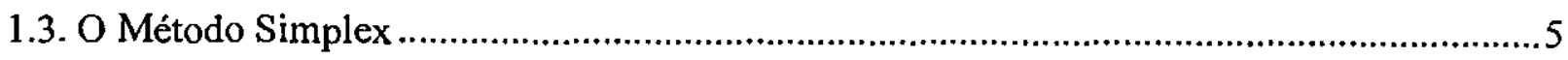

1.3.1. Introdução

1.3.2. Método Primal Simplex ......................................................................................

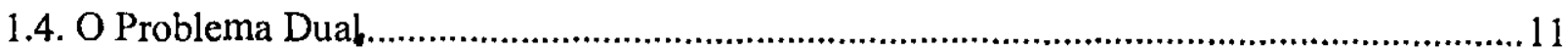

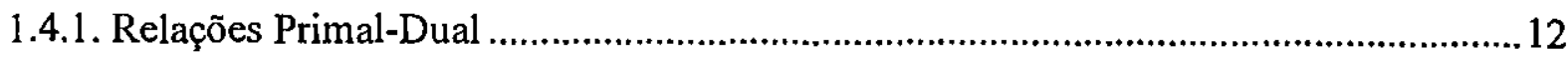

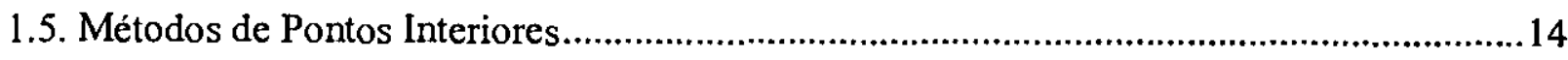

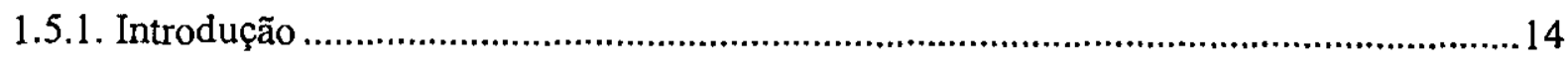

1.5.2. Os Métodos de Pontos Interiores .............................................................................15

Capítulo 2 - A Eficiência Computacional do Método Simplex .........................21

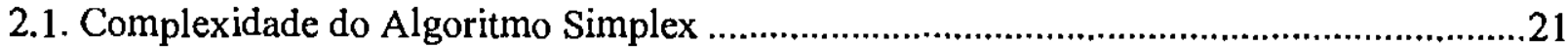

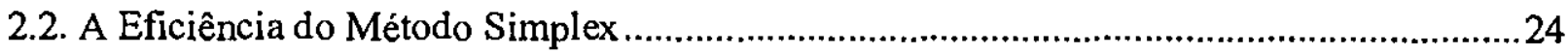

2.3. Implementações do Método Simplex...............................................................................25

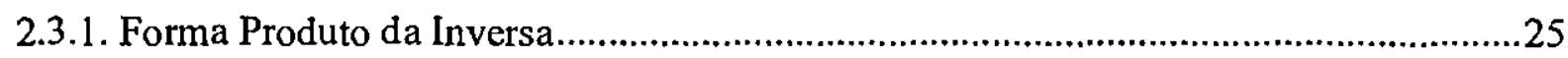

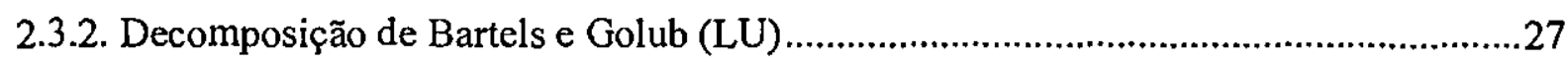

2.4. Programação Linear Aplicada ao Problema de Corte ............................................................30 
Capítulo 3 - Variantes do Método Simplex .35

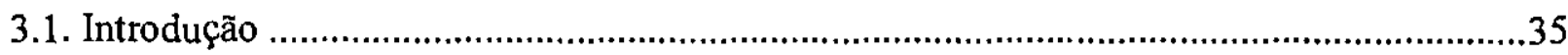

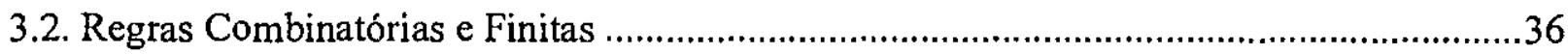

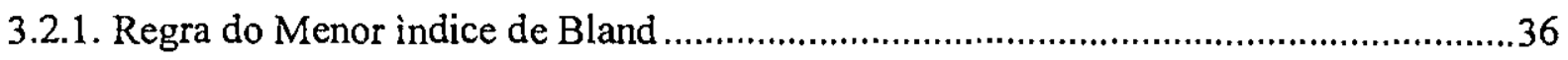

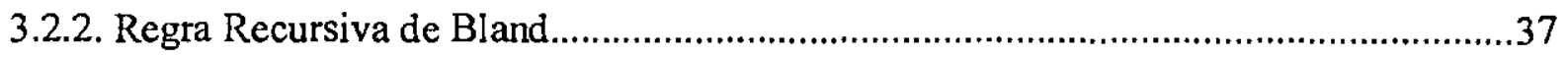

3.3. O Algoritmo de Anstreicher e Terlaky .............................................................................

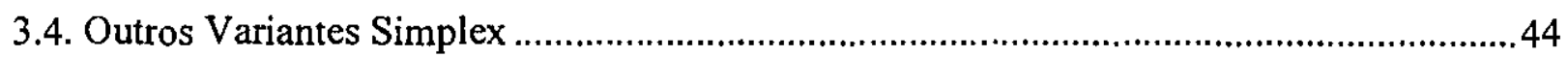

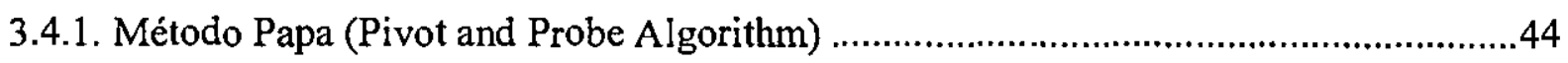

3.4.2. Nova Regra Primal e Dual de Pivoteamento …………………….............................46

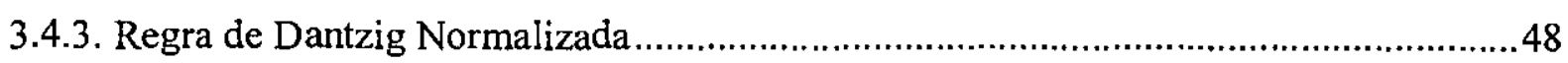

Capítulo 4 - Programa Linear com Variáveis Canalizadas................................52

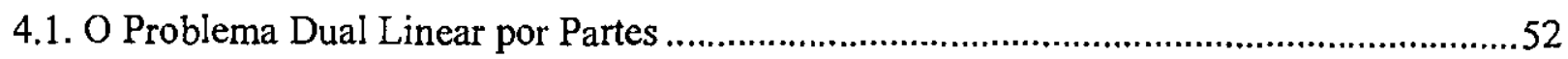

4.2. Método Dual Símplex para Variáveis Canalizadas..........................................................5

Capítulo 5 - Resultados Computacionais.......................................................59

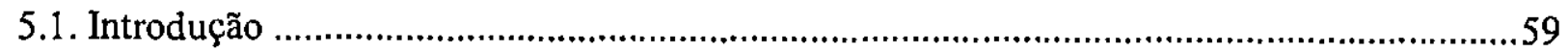

5.2. O Método Simplex Aplicado ao Problema de Corte ..........................................................59

5.3. Método Dual Simplex ...................................................................................................62.

5.4. Método Primal Simplex Revisado (Forma Produto da Inversa) .........................................64

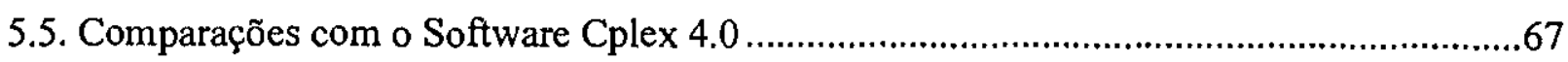

Capítulo 6 - Conclusões e Propostas Futuras...................................................69

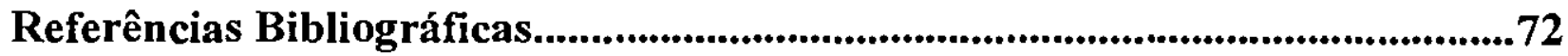




\section{Resumo}

Um problema de otimização linear consiste no problema de maximizar ou minimizar uma função linear sujeita a um conjunto de restrições lineares. Tal problema é, sem dúvidas, o modelo de otimização mais freqüentemente usado seja em planejamento da produção, alocação de recursos, bem como em muitos outros aspectos da tomada de decisão em indústrias, economia, transportes, engenharias, etc. Nesse trabalho o Método Simplex e alguns de seus variantes são investigados com ênfase na compreensão do seu desempenho computacional. 


Abstract

A linear optimization problem consists of maximizing or minimizing a linear function subject to linear constraints. Such optimization problem is, indeed, the most frequently used in production planning, resources allocation, as well as in many other aspects of decision making in industries, economy, transports, engineerings, etc. In this work the Simplex Method and some of its variants are investigated with emphasis on understanding their computational performances. 


\section{Organização do Texto}

No capítulo I fizemos um breve histórico da otimização linear, introduzimos alguns conceitos geométricos e apresentamos o método simplex primal em sua forma clássica, passo a passo, dando ainda uma pequena abordagem da teoria de dualidade, e por fim, fazemos uma breve citação sobre os métodos de pontos interiores, pois estes são objetos de intensa pesquisa para a programação linear, embora não seja o enfoque deste trabalho.

O capítulo II inicialmente trata da complexidade computacional do método simplex e sua eficiência em problemas de programação linear obtidos de aplicações práticas ou gerados aleatoriamente. Revemos um estudo sobre algumas questões relacionadas à implementação do método para um melhor desempenho do mesmo, como o método simplex revisado e a decomposição LU das matrizes básicas, proposta por Bartles e Golub (1969). Na última seção descrevemos brevemente uma aplicação ao problema de corte de estoque a título de ilustração do folclore, para verificarmos a eficiência do método simplex, que para tal realizamos alguns testes computacionais que estão no capítulo $\mathrm{V}$.

No capítulo III descrevemos de uma maneira simples e direta, não aprofundando muito sobre cada uma delas, as duas regras de Bland (1977). Apresentamos ainda alguns métodos do tipo simplex, importantes, colhidos da literatura, como o algoritmo Monotonic Build-Up (MBU) de Antreicher e Terlaky (1994).

O capitulo IV trata de um tipo específico de um problema de programação linear com variáveis canalizadas. Para a resolução deste tipo de problema, a abordagem é feita pelo método dual simplex linear por partes, Arenales (1984). 
O capítulo $\mathrm{V}$ é dedicado basicamente aos resultados computacionais obtidos das implementações feitas' durante o processo de desenvolvimento deste trabalho. Primeiramente, os testes foram realizados para o problema de corte de estoque, onde o método simplex teve um comportamento muito atraente. Em seguida os resultados são para o método dual simplex descrito no capítulo IV, onde também são feitas algumas comparações com o software Cplex em termos de número de iterações e tempo de processamento. E ainda temos alguns resultados para o método simplex revisado.

Finalmente no capítulo VI, apresentamos as conclusões do trabalho desenvolvido e destacamos alguns temas relacionados a otimização linear, tendo como base essa dissertação, para trabalhos futuros. 


\section{Capítulo I}

\section{Introdução}

\subsection{Histórico}

A história do aparecimento e evolução da programação linear registra a convivência com situações que estimulam a procura de métodos para otimizar o aproveitamento de recursos escassos, visando compartilhá-los entre atividades competitivas (Bazaraa, 1990). Embora de origem menos recente, convencionou-se como início da pesquisa operacional, o período da segunda grande guerra, quando problemas cstratćgicos e táticos de origem militares forain solucionados.

Após esse período, percebeu-se a possibilidade de aplicar un enfoque semelhante a problemas do cotidiano. As grandes organizações, como as companhias de petróleo, foram as primeiras a adotar o novo conjunto de metodologias para a resolução de seus problentas de decisão, crescendo o uso da programação linear no planejamcnto da produção em larga escala. O sucesso dos métodos justificava pesados investimentos, pelas corporações de grande porte, na busca de novos processos. Mais tarde, pecuencnas companhias puderam usufruir do conhecimento acumulado sem investir pesadamente em pesquisa. A programação linear, como instrumento de planejamento e gestão otima de sistcmas é o tópico mais difundido e utilizado da pesquisa operacional, introduzindo uma inovação ao permitir o aumento da produtividade sob as tecnologias existentes pelo aprimoramento da organização dos processos produlivos. 
A programação ou otimização linear consiste na minimização ou maximização de uma função linear, definida sobre um domínio descrito por equações e/ou inequações lineares, isto é:

$$
\begin{aligned}
& \text { Minimizar } Z(\mathbf{x})=\mathbf{c}^{\mathbf{T}} \mathbf{x} \\
& \text { Sujeito a: } \quad \mathbf{A x}=\mathbf{b} \\
& \mathbf{x} \geq \mathbf{0}
\end{aligned}
$$

onde A é uma matriz $m \times n, \mathbf{c}$ um vetor $\mathrm{n} \times 1$ e $\mathbf{b}$ um vetor $m \times 1$. A notação $\mathbf{x} \geq \mathbf{0}$ quer dizer: $\mathrm{x}_{\mathrm{j}} \geq 0, j=1, \ldots, n$. A função linear $\mathrm{Z}: \mathrm{R}^{\mathfrak{n}} \rightarrow \mathrm{R}$ é chamada função objetivo. Uma solução que satisfaça as restrições $\mathbf{A x}=\mathbf{b}, \mathbf{x} \geq \mathbf{0}$ é dita solução factível.

Resolver um problema de otimização linear, consiste em encontrar uma solução factível $x^{*}$ tal que, $Z\left(x^{*}\right) \leq Z(x)$ para todo solução factível $\mathbf{x}$. Dizemos que $\mathbf{x}^{*}$ é uma solução ótima.

O problema por programação linear foi formalizado por George B. Dantzig em 1947 que, em seguida, desenvolveu o "Método Simplex" para resolvê-lo. Desde então, um número grande de pesquisadores tem contribuido para o campo da programação linear de diferentes maneiras, incluindo desenvolvimentos teóricos, aspectos computacionais e novas aplicações.

A abordagem por programação linear desfruta de ampla aceitação por causa da habilidade de modelar importantes e complexos problemas na tomada de decisões e da capacidade de seus métodos para produzir soluções em tempo razoável. Hoje em dia, problemas com milhares de restrições e variáveis são resolvidos em microcomputadores.

No final da década de setenta, Khachian (1979) apresentou o Algoritmo dos Elipsóide para a programação linear, com tempo polinomial. Embora esse algoritmo tenha propriedades teóricas satisfatórias, o seu desempenho na prática é ruim, pois o número de iterações tende a ser muito grande e a quantidade de cálculos associados em cada iteração é muito maior comparado com o método simplex.

Karmarkar (1984) publicou um Método de Pontos Interiores capaz de encontrar uma solução ótima em tempo polinomial, para o pior caso. Além disso, o autor afirmou que seu método era muito mais rápido do que o método simplex (em alguns casos 50 vezes), o que estimulou uma avalanche de pesquisa na área de otimização linear, incluindo-se questões desafiadoras sobre a eficiência computacional de métodos do tipo simplex. Há uma observação interessante de Dantzig e Thapa (1997) de que, nos primórdios da otimização linear, Von Neumann propôs um método que hoje seria chamando de pontos interiores, porém 
as implementações feitas na época deram grande vantagem computacional ao método simplex, inibindo as pesquisas nessa direção.

Antes de apresentarmos o clássico método simplex primal de Dantzig, para um problema de programação linear, daremos brevemente alguns dos conceitos geométricos básicos, com o intuito de tornar o texto auto contido.

Em seguida é feita uma pequena descrição sobre os métodos de pontos interiores, já que esses métodos são objetos de intensa pesquisa na busca de soluções para a otimização linear, embora não seja o foco deste trabalho.

\subsection{Alguns Conceitos Geométricos}

O estudo da otimização linear requer certos conceitos e definições, que embora bem conhecido na literatura, é de conhecimento imprescindível para apresentação dos próximos capítulos de modo que decidimos incluir nesta introdução.

Um hiperplano em $\mathrm{R}^{n}$ generaliza a idéia de uma linha reta em $\mathrm{R}^{2}$ e a de um plano em $R^{3}$. Assim um conjunto $H \subset R^{n}$ é chamado um hiperplano se $H=\left\{x \in R^{n}: a^{T} x=\beta\right\}$, para algum $\mathbf{a} \in \mathrm{R}^{\mathrm{n}}-\{0\}, \beta \in \mathrm{R}$. Um hiperplano divide $\mathrm{R}^{n}$ em duas regiões, chamadas de semiespaços, e consistem nos conjuntos: $\left\{\mathbf{x} \in \mathrm{R}^{\mathrm{n}}: \mathbf{a}^{\mathrm{T}} \mathbf{x} \geq \beta\right\}$ ou $\left\{\mathbf{x} \in \mathrm{R}^{\mathrm{n}}: \mathbf{a}^{\mathrm{T}} \mathbf{x} \leq \beta\right\}$.

Um conjunto poliedral ou um poliedro em $\mathrm{R}^{n}$ é a interseção de uma família finita de hiperplanos e semi-espaços de $\mathrm{R}^{n}$, e um poliedro limitado é chamado de politopo.

Um hiperplano suporte a um poliedro $\mathrm{K}$ é um hiperplano $\mathrm{H}$ tal que $\mathrm{H} \cap \mathrm{K} \neq \varnothing, \mathrm{H} \subseteq$ $\left\{\mathbf{x} \in \mathrm{R}^{\mathrm{n}}: \mathbf{a}^{\mathrm{T}} \mathbf{x} \geq \beta\right\}$ ou $\mathrm{H} \subseteq\left\{\mathbf{x} \in \mathrm{R}^{\mathrm{n}}: \mathbf{a}^{\mathrm{T}} \mathbf{x} \leq \beta\right\}$ e $\mathrm{H} \cap \mathrm{K} \neq \varnothing$. Neste caso o conjunto $\mathrm{F}=\mathrm{H} \cap$ $K$ é chamado de uma face (autêntica) de K. Seja $k$ a dimensão de K. A face $F$ de $K$ chama-se uma faceta se $\operatorname{dim}(\mathrm{F})=k-1$, um vértice se $\operatorname{dim}(\mathrm{F})=0$, e uma aresta se $\operatorname{dim}(\mathrm{F})=1$.

Em termos do que foi definido acima, a região factível ou o conjunto de soluções factíveis do programa linear $\left\{\min \mathbf{c}^{\mathrm{T}} \mathbf{x}, A \mathbf{x} \leq \mathbf{b}\right.$ e $\left.\mathbf{x} \geq 0\right\}$, onde $\mathbf{A}$ é uma matriz $m \times x$, é o poliedro em $\mathrm{R}^{\mathrm{n}}$ definido pela interseção dos semi-espaços $\left\{\mathbf{x} \in \mathrm{R}^{\mathrm{n}}: \mathbf{a}^{\mathrm{i}} \mathbf{x} \leq \mathrm{b}_{\mathrm{i}}\right\}, i=1, \ldots, m \mathrm{e}$ $\left\{\mathrm{x} \in \mathrm{R}^{\mathrm{n}}: \mathrm{x}_{\mathrm{j}} \geq 0, j=1, \ldots, n\right\}$. Se esta região é vazia, dizemos que o problema é infactível. $\mathrm{E}$ se o problema é factível e limitado então ele tem uma solução ótima. A existência de uma solução ótima é garantida pelo teorema de Weistrass (apêndice), pois trata-se de minimizar um função contínua $(Z(x)$ é linear) num conjunto compacto (a região factível é fechada e limitada em $\mathrm{R}^{\mathrm{n}}$ ).

Um teorema fundamental da otimização linear é dado a seguir. 
Suponha que fosse feita uma lista com todas as submatrizes $\mathbf{B}_{m \times m}$ de $\mathbf{A}$. Algumas dessas submatrizes produziriam soluções básicas factíveis, enquanto outras não, a saber, as que são singulares e aquelas associadas a soluções básicas infactíveis. Portanto, o número de soluções básicas factíveis é menor ou igual a:

$$
\left(\begin{array}{l}
n \\
m
\end{array}\right)=\frac{n !}{(n-m) ! m !}
$$

$\grave{E}$ interessante observar que, embora o teorema 1.1 nos diga que basta procurar a solução ótima entre os vértices da região de factibilidade, esta procura nos remete a um conjunto de cardinalidade não-polinomial em termos dos dados do problema, podendo introduzir uma complexidade que o problema original não tem.

Definição 1.2. Chamamos o vetor $\pi \in \mathrm{R}^{m}$, dado por:

$$
\pi^{\mathrm{T}}=\mathbf{c}_{\mathrm{B}}^{\mathrm{T}} \mathbf{B}^{-1}
$$

de vetor multiplicador simplex.

Teorema 1.3. Se a partição básica $\mathbf{A}=(\mathbf{B}, \mathbf{N})$ é primal factível e $c_{j}-\pi^{\top} \mathbf{a}_{j} \geq 0$, $j=1, \ldots, n$, então a solução básica associada (definição 1.1) atual é ótima.

Observe que, pela definição de $\pi, c_{j}-\pi^{\tau} \mathbf{a}_{j}=0$ para $\mathbf{a}_{\mathrm{j}} \in \mathbf{B}$.

Considere uma solução básica primal factível não degenerada (inicial) $\mathbf{x}^{0}=\left(\begin{array}{c}\mathbf{x}_{\mathrm{B}}^{0} \\ \mathbf{x}_{\mathrm{N}}^{0}\end{array}\right)=\left(\begin{array}{c}\mathbf{B}^{-1} \mathbf{b} \\ 0\end{array}\right)$ cujo o valor da função objetivo $\mathrm{Z}\left(\mathbf{x}^{0}\right)$ é dado por:

$$
Z\left(\mathbf{x}^{0}\right)=\left(\mathbf{c}_{\mathrm{B}}^{\mathrm{T}}, \mathbf{c}_{\mathrm{N}}^{\mathrm{T}}\right)\left(\begin{array}{c}
\mathbf{B}^{-1} \mathbf{b} \\
0
\end{array}\right)=\mathbf{c}_{\mathrm{B}}^{\mathrm{T}} \mathrm{B}^{-1} \mathbf{b}=\mathbf{c}_{\mathrm{B}}^{\mathrm{T}} \mathbf{x}_{\mathrm{B}}^{0}
$$

O sistema $\mathbf{A x}=\mathbf{b}$ pode ser rescrito por:

$$
\begin{aligned}
\mathbf{x}_{B} & =\mathbf{B}^{-1} \mathbf{b}-\mathbf{B}^{-1} \mathbf{N} \mathbf{x}_{N} \\
& =\mathbf{B}^{-1} \mathbf{b}-\sum_{j \in N} \mathbf{B}^{-1} \mathbf{a}_{j} \mathbf{x}_{j} \\
& =\mathbf{x}_{B}^{0}+\sum_{j \in N} \mathbf{y}_{j} \mathbf{x}_{j}
\end{aligned}
$$

onde $\mathrm{N}$ é o conjunto dos índices das colunas (ou variáveis) não básicas $e^{*} \mathbf{y}_{\mathrm{j}}=-\mathbf{B}^{-1} \mathbf{a}_{\mathrm{j}}$. 
A solução geral do sistema $\mathbf{A x}=\mathbf{b}$ dada por (1.2) permite escrever também a função objetivo em termos das variáveis não básicas, ou seja,

$$
\begin{aligned}
Z(\mathbf{x}) & =\mathbf{c}^{\mathrm{T}} \mathbf{x} \\
& =\mathbf{c}_{B}^{\mathrm{T}} \mathbf{x}_{B}+\mathbf{c}_{N}^{\mathrm{T}} \mathbf{x}_{N} \\
& =\mathbf{c}_{B}^{\mathrm{T}}\left(\mathbf{x}_{B}^{0}+\sum_{j \in N} \mathbf{y}_{j} x_{j}\right)+\sum_{j \in N} c_{j} x_{j} \\
& =Z\left(\mathbf{x}^{0}\right)+\sum_{j \in N}\left(c_{j}-\pi^{T} \mathbf{a}_{j}\right) x_{j},
\end{aligned}
$$

o que mostra como a função objetivo, restrita ao sistema $\mathbf{A x}=\mathbf{b}$, se altera quando fazemos perturbações na solução básica factível.

Note que o problema (1.1) pode ser rescrito como:

$$
\begin{gathered}
\text { Minimizar } \quad Z(\mathbf{x})=\mathrm{Z}\left(\mathbf{x}^{0}\right)+\sum_{j \in N}\left(c_{j}-\pi \mathbf{a}_{j}\right) x_{j} \\
\text { Sujeito a: }-\sum_{j \in N} \mathbf{y}_{j} x_{j}+x_{B}=x_{B}^{0} \\
x_{j} \geq 0, j \in N, \text { e } x_{B} \geq 0
\end{gathered}
$$

Observe que as variáveis $\mathbf{x}_{B}$ simplesmente fazem o papel das variáveis de folga na equação (1.3). Assim, podemos equivalentemente escrever o problema (1.1) no espaço das variáveis não básicas:

$$
\begin{aligned}
& \text { Minimizar } \quad Z(x)=Z\left(x^{0}\right)+\sum_{j \in N}\left(c_{j}-\pi^{T} a_{j}\right) x_{j} \\
& \text { Sujeito a: }-\sum_{j \in N} y_{j} x_{j} \leq x_{B}^{0} \\
& \quad x_{j} \geq 0, j \in N
\end{aligned}
$$

Como o número de variáveis não básicas é $p=(n-m)$ podemos representar o programa linear num espaço $p$-dimensional. Isto é esperado, já que existem $p$ variáveis independentes ou $p$ graus de liberdade no sistema $\mathbf{A x}=\mathbf{b}$. O valor $\left(\mathrm{c}_{\mathrm{j}}-\pi^{\mathrm{T}} \mathbf{a}_{\mathrm{j}}\right.$ ) é referido 
algumas vezes como custo reduzido ou custo relativo, já que ele é o coeficiente da variável não básica $j$ no espaço reduzido, ou no espaço relativo às variáveis não básicas.

Definição 1.3. Chamamos de Estratégia Simplex a seguinte perturbação da solução básica inicial:

- Faça $x_{\mathrm{k}}=\varepsilon \geq 0$, para algum $k \in \mathrm{N}$ tal que $\mathrm{c}_{\mathrm{k}}-\pi^{\mathrm{T}} \mathrm{a}_{\mathrm{k}}<0$,

- Faça $x_{\mathrm{j}}=0$ para todo $j \neq k, j \in \mathrm{N}$.

A estratégia simplex produz uma nova solução dada por:

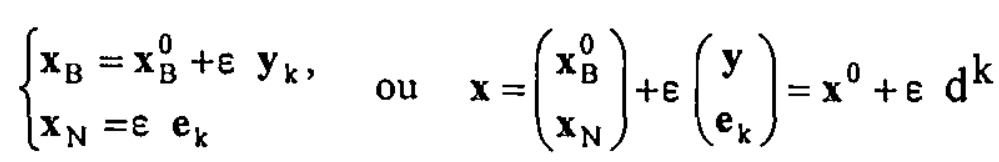

onde $\mathbf{e}_{\mathrm{k}}=(0, \ldots, 1, \ldots, 0)^{\top} \in \mathrm{R}^{n-m}$, com I na $k$-ésima componente.

Note que a direção $\mathbf{d}^{\mathrm{k}} \in \mathrm{R}^{\mathrm{n}}$, dada por $\mathbf{d}^{\mathrm{k}}=\left(\mathbf{d}_{\mathrm{B}}^{\mathrm{k}}, \mathbf{d}_{\mathrm{N}}^{\mathrm{k}}\right)^{\mathrm{T}}=\left(\mathbf{y}, \mathbf{e}_{\mathrm{k}}\right)^{\mathrm{T}}$, define uma perturbação da solução básica e é chamada direção simplex. Se a solução básica for não degenerada, ou seja, $\mathbf{x}_{\mathrm{B}}^{0}>\mathbf{0}$, de (1.4) segue que $\mathrm{d}^{\mathrm{k}}$ é uma direção factível em $\mathbf{x}^{0}$, uma vez que produzirá soluções factíveis para $\varepsilon>0$ e pequeno. Note ainda, $\mathbf{c}^{\top} \mathbf{d}^{\mathbf{d}^{k}}=\mathrm{c}_{\mathrm{k}}-\pi^{\top} \mathbf{a}_{\mathrm{k}}$.

Usando (1.4) podemos escrever o valor da função objetivo para a solução perturbada por:

$$
Z(\mathbf{x})=\mathbf{c}^{\top}\left(\mathbf{x}^{0}+\varepsilon \mathbf{d}^{k}\right)=Z\left(\mathbf{x}^{0}\right)+\varepsilon \mathbf{c}^{\top} \mathbf{d}^{k}=Z\left(\mathbf{x}^{0}\right)+\left(c_{k}-\pi^{\top} \mathbf{a}_{k}\right) \varepsilon .
$$

Considerando que o índice $k$ foi escolhido tal que $\mathbf{c}^{\mathbf{T}} \mathbf{d}^{\mathbf{k}}<0$, então de (1.5) a direção $\mathbf{d}^{\mathbf{k}}$ é de descida.

De (1.4), podemos determinar o maior valor de $\varepsilon$, mantendo $\mathbf{x}_{B} \geq \mathbf{0}$. A partir daí, temos dois casos:

- $\quad$ Se $y_{i k} \geq 0, i=1, \ldots, m$, então não há limitante para $\varepsilon, \mathrm{Z}(\mathbf{x}) \rightarrow \infty$ (a direção $\mathrm{d}^{\mathrm{k}}$ é um raio de descida) e o problema (1.1) não tem solução ótima.

- Senão o conjunto $\left\{y_{\mathrm{ik}} \mid \mathrm{y}_{\mathrm{ik}}<0, i=1, \ldots, m\right\} \neq \varnothing$. Ora, para $i$ tal que $\mathrm{y}_{\mathrm{ik}}<0$, temse:

$$
\mathrm{x}_{\mathrm{B}_{\mathrm{i}}}^{0}+\varepsilon \mathrm{y}_{\mathrm{ik}} \geq 0 \quad \Rightarrow \quad \varepsilon \leq \frac{\mathrm{x}_{\mathrm{B}_{\mathrm{i}}}^{0}}{\mathrm{y}_{\mathrm{ik}}}
$$


onde $\mathrm{x}_{\mathrm{B}_{\mathrm{i}}}^{0}$ é a $i$-ésima componente de $\mathrm{x}_{\mathrm{B}}^{0}$.

Portanto, o maior valor para $\varepsilon$ é dado por:

$\varepsilon^{0}=\frac{\mathrm{x}_{\mathrm{B}_{\mathrm{r}}}^{0}}{\mathrm{y}_{\mathrm{rk}}}=$ mínimo $\left\{\frac{\mathrm{x}_{\mathrm{B}_{\mathrm{i}}}^{0}}{\mathrm{y}_{\mathrm{ik}}} \mid \mathrm{y}_{\mathrm{ik}}<0, i=1, \ldots, m\right\}$ (critério da menor razão).

Para esta escolha de $\varepsilon$, a résima componente de $\mathbf{x}_{\mathrm{B}}$ se anula $\left(\mathrm{x}_{\mathrm{B}_{\mathrm{r}}}=\mathrm{x}_{\mathrm{B}_{\mathrm{r}}}^{0}-\frac{\mathrm{x}_{\mathrm{B}_{\mathrm{r}}}^{0}}{\mathrm{y}_{\mathrm{rk}}} \mathrm{y}_{\mathrm{rk}}=0\right)$, enquanto que apenas uma variável de $\mathrm{x}_{\mathrm{N}}$ tornou-se positiva: $x_{k}=\varepsilon^{0} \geq 0$. Isto sugere uma nova partição básica, conforme o seguinte teorema.

Teorema 1.4. Considere a nova partição $A=\left(B^{\prime}, N^{\prime}\right)$, onde a $r$-ésima coluna de $\mathbf{B}$ 'é dada por $\mathbf{a}_{\mathrm{k}} \in \mathbf{N}$. A nova partição é básica factível, cuja solução básica associada é dada por: $\mathbf{x}^{1}=\mathbf{x}^{0}+\varepsilon^{0} \mathbf{d}$.

De (1.5), podemos escolher qualquer $k$ tal que $c_{\mathrm{k}}-\pi^{\mathrm{T}} \mathbf{a}_{\mathrm{k}}<0$ para que um novo vértice $\left(\mathbf{x}^{1}\right)$ seja gerado com um valor menor para a função objetivo. Um critério (conhecido como a regra de Dantzig ou regra de máxima descida) para a escolha do índice $k$ da coluna que deverá tornar-se básica é dado por:

$$
c_{k}-\pi^{\top} \mathbf{a}_{k}=\text { mínimo }\left\{c_{j}-\pi^{\top} \mathbf{a}_{j}, j \in N\right\} .
$$

Se $c_{k}-\pi^{\mathrm{T}} \mathbf{a}_{k} \geq 0$ então, pelo teorema 1.3 , a solução básica factível é uma solução ótima.

Desta forma, enquanto a condição de otimalidade do teorema 1.3 não for verificada, podemos repetir o procedimento descrito acima. Neste caso, os conjuntos de índices B e N são atualizados através da seguinte operação:

$$
\begin{aligned}
& \mathrm{B} \leftarrow(\mathrm{B} \cup\{k\})-\{r\} \\
& \mathrm{N} \leftarrow(\mathrm{N} \cup\{r\})-\{k\}
\end{aligned}
$$

onde $\mathrm{B}$ é o conjunto dos índices das colunas (ou variáveis) básicas. Tal procedimento descreve o Método Primal Simplex.

O método primal simplex gera, a partir de uma solução inicial factível, uma seqüência de soluções factíveis do problema primal, convergente para uma solução ótima. 
Caso uma solução básica factível não esteja disponível para inicializar o método simplex, considere-se o seguinte problema artificial: (sem perda de generalidade, supomos $\left.\mathrm{b}_{\mathrm{i}} \geq 0, i=1, \ldots, m\right)$.

$$
\begin{array}{rc}
\text { Minimizar } & \mathrm{Z}^{\mathrm{a}}(\mathrm{x})=\sum_{\mathrm{i}=\mathrm{n}+\mathrm{l}}^{\mathrm{n}+\mathrm{m}} \mathrm{x}_{\mathrm{i}} \\
\text { Sujeito a : } & \sum_{\mathrm{j}=1}^{\mathrm{n}} \mathrm{a}_{\mathrm{ij}} \mathrm{x}_{\mathrm{j}}+\mathrm{x}_{\mathrm{n}+\mathrm{i}}=\mathrm{b}_{\mathrm{i}} \quad i=1, \ldots, m \\
\mathrm{x}_{\mathrm{j}} \geq 0, j=1, \ldots, n, \mathrm{x}_{\mathrm{n}+\mathrm{i}} \geq 0, i=1, \ldots, m
\end{array}
$$

Teorema 1.5. Seja $\left(\mathbf{x}_{1}^{*}, \ldots, \mathbf{x}_{n+m}^{*}\right)$ uma solução ótima do problema artificial. Então $\left(\mathbf{x}_{1}^{*}, \ldots, \mathbf{x}_{n+m}^{*}\right)$ é uma solução factível para o programa original, se e somente se, $\left(x_{n+1}^{*}=\ldots=x_{n+m}^{*}=0\right)$.

O problema artificial tem uma solução básica factível inicial óbvia (a matriz identidade, fornecida pelas colunas das variáveis artificiais), a partir da qual o algoritmo simplex inicia-se. Entretanto, nem sempre $x^{*}=\left(x_{1}^{*}, \ldots, x_{n+m}^{*}\right)$ é uma solução básica factível para o problema original. Isto acontece porque ao término da fase $\mathrm{I}$, a base pode conter alguma variável artificial nula, digamos $\mathrm{x}_{n+1}^{*}=0$, portanto uma degeneração. Com isto, a solução encontrada ao final da fase I, é factível, mas não é básica factível para o programa linear original. Ocorrências dessa natureza não impedem o uso da solução encontrada para gerar, a partir dela, uma solução básica factível para o problema original. Em geral, isto é feito pela substituição da variável artificial básica por outra variável não básica do problema original, com valor zero, por força da degeneração. A impossibilidade desta substituição significa que $i$-ésima restrição é combinação linear das demais e, portanto, pode ser eliminada do problema.

É dado a seguir o algoritmo primal simplex.

\section{Algoritmo Primal Simplex}

\section{Fase I}

Encontre uma partição básica primal-factível: $A=(B, N)$.

Faça $P A R E=F A L S O, I T=0$.

$\{$ A variável lógica PARE será falso até que a condição de otimalidade seja verificada e IT indica o número da iteração atual\} 


\section{Fase II}

Enquanto NÃO PARE faça:

1. Determine a solução básica primal factível: $\mathbf{x}_{\mathbf{n}}=\left.\mathbf{B}\right|^{-1} \mathbf{b}$.

2. Teste a otimalidade

2.1 Determine a solução básica đual: $\pi^{\top}=\mathrm{c}_{\mathrm{B}} \mathrm{B}^{-1}$.

2.2 Determine o menor custo relativo:

$$
\left(\sim_{k}\right)-\pi^{\top} \mathbf{a}_{\mathbf{k}}=\operatorname{minimo}\left\{c_{j}-\pi^{\top} \mathbf{a}_{j}, j \in N\right\} \text { //Regra de Dantzig // }
$$

2.3 Se $c_{\mathrm{j}}-\pi^{\mathrm{T}} \mathbf{a}_{\mathrm{j}} \geq 0 \forall j \in \mathrm{N}$, então a solução na iteração IT é ótima.

$P A R E=V E R D A D E$.

3. Senão đetermine as coordenadas básicas da direção simplex: $\mathbf{y}=-\mathbf{B}^{-1} \mathbf{a}_{\mathbf{k}}$

4. Determine o passo:

$$
\varepsilon^{0}=\frac{\mathrm{x}_{\mathrm{B}_{r}}^{0}}{\mathrm{y}_{r}}=\operatorname{minimo}\left\{\frac{\mathrm{x}_{\mathrm{B}_{\mathrm{i}}}^{0}}{\mathrm{y}_{\mathrm{ik}}} \mid \mathrm{y}_{\mathrm{ik}}<0, i=1, \ldots, m\right\} .
$$

Se $\mathrm{y}_{i k} \geq 0, i=1, \ldots, m$, então o problema não tem solução ótima finita. $P A R E=V E R D A D E$.

5. Atualize a partição básica: $\mathrm{a}_{\mathrm{B}_{r}} \leftrightarrow \mathrm{a}_{\mathrm{k}}, I T \leftarrow I T+l$.

\subsection{O Problema Dual}

Associado a cada problema de programação linear, existe um outro também linear chamado problema dual. O programa linear dual possui muitas propriedades importantes com relação ao problema original. Existem duas formas importantes (definições) de dualidade: a forma canônica e a forma pađrão. Essas duas formas são completamente equivalentes. Elas surgiram respectivamente da forma de representação canônica e padrão de problemas de programação linear.

Suponha que o programa linear primal é dado na forma canônica:

$$
\begin{array}{ll}
\text { Minimizar } & \mathbf{c}^{\mathrm{T}} \mathbf{x} \\
\text { Sujeito a: } & \mathbf{A x} \geq \mathbf{b} \\
& \mathbf{x} \geq \mathbf{0}
\end{array}
$$

então o programa linear dual é definido por:

$$
\begin{aligned}
& \text { Maximizar } \mathbf{b}^{\mathbf{T}} \mathbf{w} \\
& \text { Sujeito a: } \mathbf{A}^{\mathrm{T}} \mathbf{w} \leq \mathbf{c} \\
& \mathbf{w} \geq \mathbf{0}
\end{aligned}
$$


Note que há exatamente uma variável dual para cada restrição primal e exatamente uma restrição dual para cada variável primal.

Outra definição equivalente da dualidade pode ser dada com o problema primal na seguinte forma padrão:

$$
\begin{array}{ll}
\text { Minimizar } & \mathbf{c}^{\boldsymbol{T}} \mathbf{x} \\
\text { Sujeito a: } & \mathbf{A x}=\mathbf{b} \\
\mathbf{x} & \geq \mathbf{0}
\end{array}
$$

então o programa linear dual é definido por:

$$
\begin{aligned}
& \text { Maximizar } \mathbf{b}^{\top} \mathbf{w} \\
& \text { Sujeito a: } \quad \mathbf{A}^{\mathbf{T}} \mathbf{w} \leq \mathbf{c} \\
& \mathbf{w} \quad \text { irrestrito de sinal. }
\end{aligned}
$$

\subsubsection{Relações Primal-Dual}

Considerando-se a definição de dualidade na sua forma canônica, dada anteriormente, conduz a muitas relações importantes entre os problemas primal de dual de programação linear. Assim temos os teoremas e corolários abaixo:

Teorema 1.6. O dual do dual é o primal.

Teorema 1.7. Sejam $x^{*}$ e $w^{*}$ soluções factíveis de $\left(P_{l}\right)$ e $\left(D_{1}\right)$ (analogamente $\left(P_{2}\right)$ e $\left(D_{2}\right)$ ) respectivamente, então $\mathbf{c x}^{*} \geq \mathbf{w b}^{*}$.

Isto é, a função objetivo do programa dual fornece um limitante inferior para a função objetivo do problema primal.

Corolário 1.1. Se $\mathbf{x}^{*}$ e $\mathbf{w}^{*}$ são soluções factíveis de $\left(P_{1}\right)$ e $\left(D_{1}\right)$ (analogamente $\left(P_{2}\right)$ e $\left.\left(\mathrm{D}_{2}\right)\right)$ respectivamente, são tais que $\mathbf{c x}^{\bullet}=\mathbf{w b}^{*}$ então, elas são soluções ótimas.

Corolário 1.2. Se o valor da função objetivo de um dos problemas não for limitada, então outro problema (associado) não tem uma solução factível. 
Esta propriedade não é simétrica, pois pode-se ter um problema infactível no qual o outro problema é também infactível.

Um outro teorema que não é demonstrado simplesmente a partir da definição do problema dual (normalmente usa-se o lema de Farkas para demonstração, veja Bazaraa, 1990) é enumerado a seguir.

Teorema 1.8. Se um problema tem solução ótima então o outro também tem solução ótima e os objetivos coincidem, isto é, se $\mathbf{x}^{*}$ é solução ótima primal então existe $\mathbf{w}^{*}$, solução ótima dual e $\mathbf{c} \mathbf{x}^{*}=\mathbf{b}^{\mathrm{T}} \mathbf{w}^{*}$.

Uma demonstração alternativa pode ser obtida pela finitude do método simplex, isto é, existe $\pi$ (vetor multiplicador) tal que:

$$
\mathrm{c}_{\mathrm{j}}-\pi \mathbf{a}_{\mathrm{j}} \geq 0 j=1, \ldots, n
$$

isto é equivalente a:

$$
\mathbf{A}^{\mathrm{T}} \pi \leq \mathbf{c}
$$

ou seja, $\pi$ é uma solução dual factivel. Além disso, supondo $\mathbf{x}^{*}=\left(\begin{array}{c}\mathbf{x}_{\mathrm{B}}^{*} \\ 0\end{array}\right)$ uma solução básica (sem perda de generalidade teorema 1.1) onde $\mathbf{x}_{\mathrm{B}}^{*}=\mathbf{B}^{-1} \mathbf{b}$, temos:

$$
\mathbf{c}^{T} \mathbf{x}^{*}=\mathbf{c}_{\mathrm{B}} \mathbf{B}^{-1} \mathbf{b}=\pi \mathbf{b},
$$

pelo corolário 1.1 , segue que $\pi$ é solução ótima dual.

Combinando os resultados dos teoremas e corolários acima, obtem-se o seguinte teorema:

\section{Teorema 1.9. (Teorema fundamental da Dualidade)}

Considere o par de problemas (Pl) e (D1) (analogamente $\left(\mathrm{P}_{2}\right)$ e $\left(\mathrm{D}_{2}\right)$ ). Uma e somente uma das afirmativas seguintes é verdadeira.

a) Nenhum dos problemas tem solução factível.

b) Um problema tem a função objetivo ilimitada, o outro problema associado é infactível.

c) Ambos possuem soluções ótimas $\mathbf{x}^{*} \mathrm{e} \mathbf{w}^{*} \operatorname{com} \mathbf{c x ^ { * }}=\mathbf{w b}^{*}$. 
Vamos apresentar agora, um importante teorema da teoria de dualidade, que é o teorema das folgas complementares, relacionando os problemas primal e dual.

Teorema 1.10. (Teorema das Folgas Complementares) Dado o par de problemas $\left(P_{1}\right)$ e $\left(D_{1}\right)$. Sejam $x^{*}$ e w* quaisquer soluções factíveis para $\left(P_{1}\right)$ e $\left(D_{1}\right)$ (analogamente $\left(P_{2}\right)$ e $\left(D_{2}\right)$ ) respectivamente, uma condição necessária e suficiente para que sejam soluções ótimas é que elas satisfaçam as relações abaixo:

$$
\begin{aligned}
& \left(\mathrm{c}_{\mathrm{j}}-\mathrm{w}^{*} \mathrm{a}_{\mathrm{j}}\right) \mathrm{x}_{\mathrm{j}}^{*}=0 \quad j=1, \ldots, n \\
& \mathrm{w}_{\mathrm{i}}^{*}\left(\mathrm{a}^{\mathrm{i}} \mathrm{x}^{*}-\mathrm{b}_{\mathrm{i}}\right)=0 \quad i=1, \ldots, m .
\end{aligned}
$$

Obviamente, este teorema indica que pelo menos uma das parcelas em cada equação deve ser zero. Com isto temos a seguintes conclusões:

- $\mathrm{Na}$ otimalidade se uma variável num problema é estritamente positiva, então a restrição correspondente no outro problema deve ser ativa, ou seja, ser satisfeita com igualdade. E se uma restrição num problema não é ativa, então a variável correspondente no outro problema deve ser zero.

\subsection{Métodos de Pontos Interiores}

\subsubsection{Introdução}

Esta seção sobre método de pontos interiores, está baseada no trabalho de Freund e Mizuno (1996), que apresentam um panorama de tais métodos no campo da otimização linear.

Métodos de pontos interiores em programação matemática têm sido a área mais dinâmica de pesquisa em otimização desde o desenvolvimento do método simplex para programação linear. Até meados da década de noventa, métodos de pontos interiores atraiu alguns dos melhores pesquisadores em pesquisa operacional, matemática aplicada, e ciência da computação. De acordo com Freund e Mizuno (1996), aproximadamente 2000 artigos foram escritos no assunto, seguindo o trabalho Karmarkar (1984).

Métodos de pontos interiores têm mudado permanentemente o panorama da teoria, da prática e da computação em programação matemática. Programação linear não é mais sinônimo do clássico método simplex, e muitos pesquisadores tendem a ver agora a 
programação linear mais como um caso especial de programação não linear devido ao desenvolvimento desses métodos.

Segundo Freund e Mizuno, a "pedagogia" de métodos de pontos interiores atrasou sua pesquisa até pouco tempo, parcialmente porque estes métodos (i) usam ferramentas matemáticas mais avançadas do que métodos do tipo simplex, (ii) a análise matemática deles é tipicamente muito mais complicada, e (iii) os métodos são menos receptivo a intuição geométrica. Até pouco tempo, os educadores lutaram com a questão de como e onde introduzir métodos de pontos interiores no currículo de programação linear e não linear e como convincentemente expor tais métodos para estudantes (e os pesquisadores da mesma categoria). Como a pesquisa em métodos de pontos de interiores para programação linear se acalmou (e a pesquisa em pontos interiores para programação não linear aqueceu), vários projetos de livro novos em programação linear e/ou métodos de pontos interiores apareceram recentemente no cenário, prometendo superar estas dificuldades.

\subsubsection{Os Métodos de Pontos Interiores}

Para introduzir tais métodos para otimização linear considere o problema de programação linear na forma padrão:

$$
\begin{array}{r}
\text { Minimizar } \mathbf{c}^{\mathbf{T}} \mathbf{x} \\
\text { Sujeito a: } \mathbf{A x}=\mathbf{b} \\
\mathbf{x} \geq \mathbf{0}
\end{array}
$$

onde A é uma matriz $m \times n$, e o problema dual é da seguinte forma (incluindo-se as variáveis de folga):

$$
\begin{aligned}
& \text { Maximizar } \mathbf{b}^{\mathrm{T}} \mathbf{y} \\
& \text { Sujeito } a: \mathbf{A}^{\mathrm{T}} \mathbf{w}+\mathbf{s}=\mathbf{c} \\
& \quad \mathbf{s} \geq \mathbf{0}
\end{aligned}
$$

Dada uma solução factível $\mathbf{x}$ de (P) e uma solução factível (w, s) de (D), o gap de dualidade é facilmente expresso por: 


$$
\begin{aligned}
c^{\mathrm{T}} x-b^{\mathrm{T}} w & =c^{\mathrm{T}} x-x^{\mathrm{T}} A^{\mathrm{T}} w \\
& =x^{\mathrm{T}}\left(c-A^{\mathrm{T}} w\right) \\
& =x^{\mathrm{T}} s \geq 0
\end{aligned}
$$

(a desigualdade decorre do teorema 1.8).

O gap de dualidade é zero se, e somente se, $\mathbf{x}$ é ótimo em (P) e $\mathbf{w}$ é ótimo em (D). Assim, o gap de dualidade serve como uma boa medida para verificar o quanto as soluções factíveis $\mathbf{x}$ e w estão próximo das soluções ótimas para (P) e (D).

As condições do teorema 1.10. (teorema das folgas complementares) para o par (P) - (D) podem ser equivalentemente escritas por uma das propriedades:

1. $\left(s^{*}\right)^{\mathrm{T}} \mathbf{x}^{*}=0$;

2. $\mathrm{x}_{\mathrm{j}}^{*} \mathrm{~s}_{\mathrm{j}}^{*}=0 \quad \forall j=1, \ldots, n$;

3. Se $\mathrm{s}_{\mathrm{j}}^{*}>0$ então $\mathrm{x}_{\mathrm{j}}^{*}=0$.

Utilizaremos a seguinte notação mais conveniente para o desenvolvimento de métodos de pontos interiores. Uma solução factível $\mathbf{x}$ de $(\mathrm{P})$ é interior se $\mathbf{x}>\mathbf{0}$ e uma solução factível $(\mathbf{w}, \mathbf{s})$ de (D) é interior se $\mathbf{s}>\mathbf{0}$. Seja $\mathbf{e}=(1, \ldots, 1)^{\mathrm{T}}$. Uma matriz $\mathbf{X}$ é definida como sendo a matriz diagonal $n \times n$, onde cada elemento da diagonal é justamente as componentes do vetor x, isto é:

$$
\mathbf{X}=\left(\begin{array}{cccc}
\mathrm{x}_{1} & 0 & \cdots & 0 \\
0 & \mathrm{x}_{2} & \cdots & 0 \\
\vdots & \vdots & \ddots & \vdots \\
0 & 0 & \cdots & \mathrm{x}_{\mathrm{n}}
\end{array}\right)
$$

Note que $\mathbf{X e}=\mathbf{x}, \mathbf{e}$ (supondo $\mathbf{x}>\mathbf{0}) \mathbf{X}^{-1} \mathbf{e}=\left(1 / \mathrm{x}_{1}, \cdots, 1 / \mathrm{x}_{\mathrm{n}}\right)^{\boldsymbol{\tau}}$, e que $\mathbf{X}$ e $\mathbf{X}^{-1}$ são matrizes simétricas definidas positivas.

Nas opiniões de alguns autores, a maioria dos métodos de pontos interiores estão numa das três principais categorias: Métodos Afim-Escala, Métodos de Redução Potencial, e Métodos da Trajetória Central. Resumiremos brevemente estas três categorias.

a) Métodos Afim-Escala. A estratégia básica do algoritmo Afim-Escala é: dada uma solução interior $\overline{\mathbf{x}}$ de $(\mathrm{P})$, construa um elipsóide simples local $\mathrm{E}_{\overline{\mathbf{x}}}$ na região factível de $(\mathrm{P})$ centrado 
em $\overline{\mathbf{x}}$. Então, otimize a função objetivo $\mathbf{c}^{\top} \mathbf{x}$ sobre $E_{\overline{\mathbf{x}}}$, e use a direção resultante com um passo apropriado para definir um nova solução. As particularidades desta estratégia são:

Dada solução interior $\overline{\mathbf{x}}$ de $(\mathrm{P})$, o Elipsóide em $\overline{\mathbf{x}}$ é definido como:

$$
\mathrm{E}_{\overline{\mathbf{x}}}=\left\{\mathbf{x} \in \mathrm{R}^{\mathrm{n}} \mid \mathbf{A x}=\mathbf{b},(\mathbf{x}-\overline{\mathbf{x}})^{\mathrm{T}} \overline{\mathbf{X}}^{-2}(\mathbf{x}-\overline{\mathbf{x}}) \leq 1\right\}
$$

(esta vizinhança de $\overline{\mathbf{x}}, \mathrm{E}_{\bar{x}}$ está contida na região factível de (P) desde que $\overline{\mathbf{x}}$ é interior.) $A$ direção Afim em $\overline{\mathbf{x}}$ é então a solução do seguinte problema:

$$
\begin{aligned}
& \text { Minimizar } \mathbf{c}^{\mathbf{T}} \mathbf{d} \\
& \text { Sujeito a : } \mathbf{A d}=\mathbf{0} \\
& \mathbf{d}^{\mathbf{T}} \overline{\mathbf{X}}^{-2} \mathbf{d} \leq 1
\end{aligned}
$$

Note que $(\mathrm{P} \overline{\mathrm{x}})$ é um problema convexo com todas as restrições lineares exceto para uma restrição quadrática convexa. Pode ser resolvido analiticamente, a partir das condições necessárias e suficientes de $1^{\text {a }}$ ordem de Karush-Kuhn-Tucker.

Segundo Freund e Mizuno (1996), este algoritmo é atraente devido a sua simplicidade e seu bom desempenho na prática. (Porém, seu desempenho é bastante sensível para o ponto inicial do algoritmo.) Não existe qualquer resultado sobre complexidade computacional, mas há fortes suspeitas que o algoritmo seja exponencial no pior caso. (Freund e Mizuno, 1996).

Alguns variantes/extensões no algoritmo básico Afim-Escala é o algoritmo AfimEscala Dual (desenvolvido para desigualdades, veja Adler et al. 1986 e Balbo 1991), como também várias versões que trabalham simultaneamente no primal e no dual, usando uma variedade de construções do elipsóide no espaço primal e dual.

b) Métodos de Redução Potencial. Métodos de redução potencial são tipicamente projetado para encontrar soluções melhores para o seguinte problema de otimização não linear:

Problema de Redução Potencial (PRP)

$$
\begin{aligned}
& \text { Minimizar } f(x, w, s)=q \ln \left(\mathbf{c}^{\mathrm{T}} \mathbf{x}-\mathbf{b}^{\mathrm{T}} \mathbf{w}\right)-\sum_{\mathrm{j}=1}^{\mathrm{n}} \ln \left(\mathrm{x}_{\mathrm{j}}\right) \\
& \text { Sujeito a : } \quad \mathbf{A x}=\mathbf{b} \\
& \mathbf{x}>\mathbf{0} \\
& \mathbf{A}^{\mathrm{T}} \mathbf{w}+\mathbf{s}=\mathbf{c} \\
& \mathbf{s} \geq \mathbf{0}
\end{aligned}
$$


onde a função objetivo $\mathrm{f}(\mathbf{x}, \mathbf{w}, \mathbf{s})$ é chamada a função potencial, e $q$ um parâmetro. Foi este tipo de problema que Karmarkar trabalhou em sua publicação. Note que a "primeira parte" da função potencial é $q$ vezes o logaritmo do gap de dualidade, e deseja-se conduzir esta parte para $-\infty$. A Segunda parte da função potencial é a função barreira logaritma introduzida para "repelir" soluções na fronteira da região factível.

O algoritmo original de Karmarkar (1984) usou uma forma muito específica de (PRP) e também ó procedimento de transformações projetiva no algoritmo e na prova de garantia do desempenho do algoritmo (Gonzaga 1992). Segundo Freund e Mizuno (1996), apesar da mística original, transformaçð̃es projetivas não são necessárias para algoritmos de redução potencial, seja na teoria como na prática, porém, nas opiniões de alguns autores, a estrutura de trabalho de transformaç̃es projetivas é, contudo, de suprema importância, pelo menos conceitualmente, na compreensão de métodos de pontos interiores em geral.

Em geral, todo método de redução potencial tem como objetivo conduzir a função potencial para - $\infty$ através de uma variedade de algoritmos primal, dual ou primal-dual. Quase todos métodos de redução de potencial desfrutam do desempenho garantido de complexidade polinomial. Métodos de redução de potencial não receberam muita atenção em termos de testes computacionais, devido talvez às dificuldades anteriores (que foram superadas) de aplicar métodos de redução potencial em um ambiente combinando Fase I e Fase II. Para um estudo aprofundado sobre esses métodos, referencia-se os trabalhos de Anstreicher (1996) e Todd (1997).

c) Métodos de Trajetória Central. Métodos de pontos interiores baseados na trajetória central são, sem dúvida, os mais úteis na teoria, e o mais usado na prática. (Freund e Mizuno (1996) citam em sua publicação, que um dos principais pesquisadores da área se referiu à trajetória central até como "o mais importante objeto na teoria moderna de otimização"). A trajetória central do problema linear $(\mathrm{P})$ é obtida como a solução para uma versão compensada de $(\mathrm{P})$, onde um termo barreira logarítmica parametrizada é somado à função objetivo. Considere o problema de barreira logarítmico BP $(\mu)$ parametrizado pelo parâmetro de barreira positiva $\mu$ :

$$
\begin{array}{cc}
\text { Minimizar } & \mathbf{c}^{\mathrm{T}} \mathbf{x}-\mu \sum_{\mathbf{j}=1}^{\mathrm{n}} \ln \left(\mathbf{x}_{\mathrm{j}}\right) \\
\text { Sujeito a: } & \mathbf{A x}=\mathbf{b} \\
& \mathbf{x}>\mathbf{0}
\end{array}
$$


As condições necessárias e suficientes de $1^{\mathrm{a}}$ ordem de Karush-Kuhn-Tucker para $\mathrm{BP}(\mu)$ são:

$$
\left\{\begin{array}{l}
\mathbf{A x}=\mathbf{b}, \mathbf{x}>\mathbf{0} \\
\mathbf{c}-\mu \mathbf{X}^{\cdot \mathrm{l}} \mathbf{e}=\mathbf{A}^{\mathrm{T}} \mathbf{w}
\end{array}\right.
$$

Se definirmos $\mathbf{s}=\mu \mathbf{X}^{-1} \mathbf{e}$, então podemos rescrever essas condições de otimalidade como;

$$
\left\{\begin{array}{l}
\mathbf{A x}=\mathbf{b}, \mathbf{x}>\mathbf{0} \\
\mathbf{A}^{\mathrm{T}} \mathbf{w}+\mathbf{s}=\mathbf{c}, \mathbf{s}>\mathbf{0} \\
\mathbf{X S e}-\mu \mathrm{e}=\mathbf{0}
\end{array}\right.
$$

Seja $(\mathbf{x}(\mu), \mathbf{w}(\mu), \mathbf{s}(\mu))$ a solução para o sistema (1.8) para um dado parâmetro $\mu$ positivo. Então o conjunto $\Gamma=\{(\mathbf{x}(\mu), \mathbf{w}(\mu), \mathbf{s}(\mu))$ tal que $\mu>0\}$ é definido como trajetória central do problema linear (P). Das duas primeiras equações do sistema (1.8), vemos que uma solução (x, w, s) ao longo da trajetória central é estritamente factível para o problema primal e para o problema dual, e que o gap de dualidade na trajetória central é $\mathbf{x}^{\mathrm{T}} \mathrm{S}=\mathbf{e}^{\mathrm{T}} \mathbf{X S e}=\mu \mathbf{e}^{\mathrm{T}} \mathbf{e}=$ $\mu n$, que segue da terceira equação do sistema (1.8). Substituindo esta equação na terceira do sistema (1.8), obtemos a seguinte caracterização da trajetória central, livre de parâmetro que é equivalente a anterior:

$$
\left\{\begin{array}{l}
\mathbf{A x}=\mathbf{b}, \mathbf{x}>\mathbf{0} \\
\mathbf{A}^{\mathrm{T}} \mathbf{w}+\mathbf{s}=\mathbf{c}, \mathbf{s}>\mathbf{0} \\
\mathrm{XSe}-\left(\mathbf{x}^{\mathrm{T}} \mathrm{s} / n\right) \mathrm{e}=\mathbf{0}
\end{array}\right.
$$

A terceira equação no sitema (1.8) ou (1.9) não é linear e, em geral, não é possível resolver (1.8) ou (1.9) de forma fechada, exceto no caso trivial.

A estratégia na maioria dos métodos da trajetória central consiste em resolver por soluções aproximadas, ao longo da trajetória central (1.8) ou (1.9) para diminuir na sequuência o gap de dualidade (ou equivalentemente, do parâmetro de barreira $\mu$ ) que tende a zerar no limite. Há várias maneiras para realizar esta estratégia. Por exemplo, para um dado valor do gap de dualidade ou do parâmetro barreira $\mu$, pode-se escolher aproximadamente otimizar $\mathrm{BP}(\mu)$ ou, equivalentemente, resolver aproximadamente (1.7), (1.8), ou (1.9), ou resolver 
aproximadamente alguma outra caracterização equivalente da trajetória central. Também, pode-se escolher várias maneiras para resolver aproximadamente o sistema de equações não linear (o método de Newton é uma escolha óbvia, como são os métodos preditor-corrector, métodos do gradiente conjugados precondicionado, etc.). Por último, há uma liberdade considerável em desenvolver uma estratégia por reduzir o gap de dualidade (ou o parâmetro de barreira $\mu$ ) a cada iteração. (Por exemplo, encolher o gap de dualidade agressivamente parece uma idéia boa, mas também aumenta o número de iterações do método de Newton (ou outro método) que é usado para re-solucionar (aproximadamente) o novo sistema de equações não lineares.)

Um resumo dos métodos da trajetória central é dado em Jansen et al (1995), e Hertog (1994) em que faz um trabalho mais profundo sobre tais métodos. 


\section{Capítulo II}

\section{A Eficiência Computacional do Método Simplex}

A questão da eficiência do método simplex sempre foi tema de pesquisa desde sua publicação. No início, a convergência finita do método, garantida com regras que evitassem ciclagem, era suficiente para os pesquisadores. No entanto, o número máximo de iterações poderia ser muito grande. Um conjunto de relatos sobre sua eficiência em problemas práticos ou gerados de forma aleatória formou o chamado folclore do métoḍo simplex. Shamir (1987) faz uma revisão deste folclore.

\subsection{Complexidade Computacional do Algoritmo Simplex}

Considere novamente o problema primal de otimização linear na forma:

$$
\begin{aligned}
& \text { Minimizar } Z(x)=\mathbf{c}^{\mathbf{T}} \mathbf{x} \\
& \text { Sujeito a: } \quad \mathbf{A x}=\mathbf{b} \\
& x \geq 0
\end{aligned}
$$

onde A é uma matriz $m \times n$.

A especificação dos valores para $m, n, \mathbf{c}, \mathbf{A} \mathrm{e} \mathbf{b}$ define um exemplar de um problema de programação linear. O tamanho desse exemplar é representado pelos dados de entrada num computador, que são: a matriz de restrições $\mathbf{A}$ e os vetores $\mathbf{c}$ e b, cujas dimensões são. definidas pelos valores de $m$ e $n$. 
Considere um algoritmo finito que resolve um programa linear. A fim de analisar a complexidade computacional, precisamos determinar um limitante superior para o esforço necessário em resolver qualquer exemplar desse problema. Esse esforço pode ser medido em termos do número de operações elementares tais como adições, multiplicações e comparações, necessárias para resolver o problema, como uma função do tamanho do mesmo.

No algoritmo simplex, o principal passo computacional é a resolução dos sistemas básicos a ser realizado a cada iteração, que pode ser feito em $\mathrm{O}\left(\mathrm{m}^{3}\right)$. Assim, o esforço necessário para resolver (2.1), pelo algoritmo simplex pode ser medido pelo número de iterações.

A questão que se coloca é a seguinte: Qual é o número médio de iterações para resolver um problema de otimização linear? É possivel expressar este número médio como uma função de $m$ e $n$ ? (Esta função é conhecida como complexidade computacional média do algoritmo simplex).

Determinar essa função é uma questão estatística difícil de ser respondida precisamente porque os tipos de programas lineares que são obtidos de aplicações práticas não são fáceis de serem especificados. No entanto, da experiência computacional com um número grande de problemas resolvidos em vários anos (Murty, 1983), uma resposta empírica para essa questão surgiu, indicando que o número médio de iterações é uma função linear de $m$, que parece ser menor do que $3 \mathrm{~m}$.

Uma questão mais desafiante é: dado um problema de programação linear de um certo tamanho, qual é o limitante superior para o número de iterações ao resolver tal problema? Provavelmente esta questão não tenha muita importância prática, mas é teoricamente muito interessante. Esse limitante superior determina aquele que é conhecido como o pior caso da complexidade computacional (diferentemente da complexidade computacional média citada anteriormente). $\mathrm{O}$ estudo da complexidade computacional pelo pior caso fornece um limitante superior no esforço computacional, necessário para resolver qualquer programa linear pelo algoritmo simplex, como uma função do tamanho deste.

Dizemos que um algoritmo tem tempo polinomial para um problema de decisão, se pode resolver qualquer exemplar do problema usando um número de operações elementares (no caso do algoritmo simplex, o número de iterações) limitado superiormente por algum polinômio no tamanho do problema. Neste caso, diz-se que o problema pertence à classe polinomial (P).

Klee e Minty (1972) apresentaram um exemplar, para o qual o método simplex necessita de $2^{n}-1$ iterações, percorrendo todos os vértices da região factível do problema, 
onde a região factível é definida como uma distorção do hipercubo $n$-dimensional, o qual tem $2^{n}$ vértices. Matematicamente, o problema em $\mathrm{R}^{n}$ pode ser descrito da seguinte forma:

$$
\begin{aligned}
& \text { Maximizar } \quad \mathrm{x}_{\mathrm{n}} \\
& \text { Sujeito a: } \quad 0 \leq \mathrm{x}_{1} \leq 1 \\
& \varepsilon \mathrm{x}_{\mathrm{j}-1} \leq \mathrm{x}_{\mathrm{j}} \leq 1-\varepsilon \mathrm{x}_{\mathrm{j}-1} \quad \text { para } j=2, \ldots, n \\
& \mathrm{x}_{\mathrm{j}} \geq 0, j=1, \ldots, n
\end{aligned}
$$

onde $\varepsilon$ é um número racional no intervalo $(0,1 / 2)$.

Após uma transformação sutil desse problema (Bazaraa, 1990), usando a regra de Dantzig (ou regra de máxima subida, já que o objetivo é maximizar) e tendo como uma solução básica factível inicial a origem, o algoritmo simplex caminha em $2^{n}-1$ arestas desse politopo e visita todos os $2^{n}$ vértices da região factível.

Por exemplo, considere o caso $n=3$. Então o problema citado anteriormente pode ser rescrito da seguinte forma:

$$
\begin{aligned}
& \text { Minimizar }-\mathrm{x}_{3} \\
& \text { Sujeito a: } 0 \leq \mathrm{x}_{1} \leq 1 \\
& \varepsilon \mathrm{x}_{1} \leq \mathrm{x}_{2} \leq 1-\varepsilon \mathrm{x}_{1} \\
& \varepsilon \mathrm{x}_{2} \leq \mathrm{x}_{3} \leq 1-\varepsilon \mathrm{x}_{2}
\end{aligned}
$$

$\mathrm{O}$ conjunto de soluções factíveis desse problema é o cubo em $\mathrm{R}^{3}$ ligeiramente perturbado representado pela figura 1 , onde as soluções básicas são descritas abaixo:

$$
\begin{array}{ll}
x^{0}=(0,0,0) & x^{4}=(0,1,1-\varepsilon) \\
x^{1}=\left(1, \varepsilon, \varepsilon^{2}\right) & x^{5}=(1,1-\varepsilon, 1-\varepsilon(1-\varepsilon)) \\
x^{2}=(1,1-\varepsilon, \varepsilon(1-\varepsilon)) & x^{6}=\left(1, \varepsilon, 1-\varepsilon^{2}\right) \\
x^{3}=(0,1, \varepsilon) & x^{7}=(0,0,1)
\end{array}
$$

Para o caminho $\mathrm{x}^{0} \rightarrow \mathrm{x}^{1} \rightarrow \mathrm{x}^{2} \rightarrow \mathrm{x}^{3} \rightarrow \mathrm{x}^{4} \rightarrow \mathrm{x}^{5} \rightarrow \mathrm{x}^{6} \rightarrow \mathrm{x}^{7}$, notamos que a função objetivo decresce monotonamente a cada vértice percorrido e consiste no caminho simplex, segundo a regra de máxima descida. Assim, são necessárias $7=2^{3}-1$ iterações para o algoritmo simplex atingir a solução ótima. Para o problema de dimensão $n$, o método simplex faria $2^{n}-1$ iterações. 


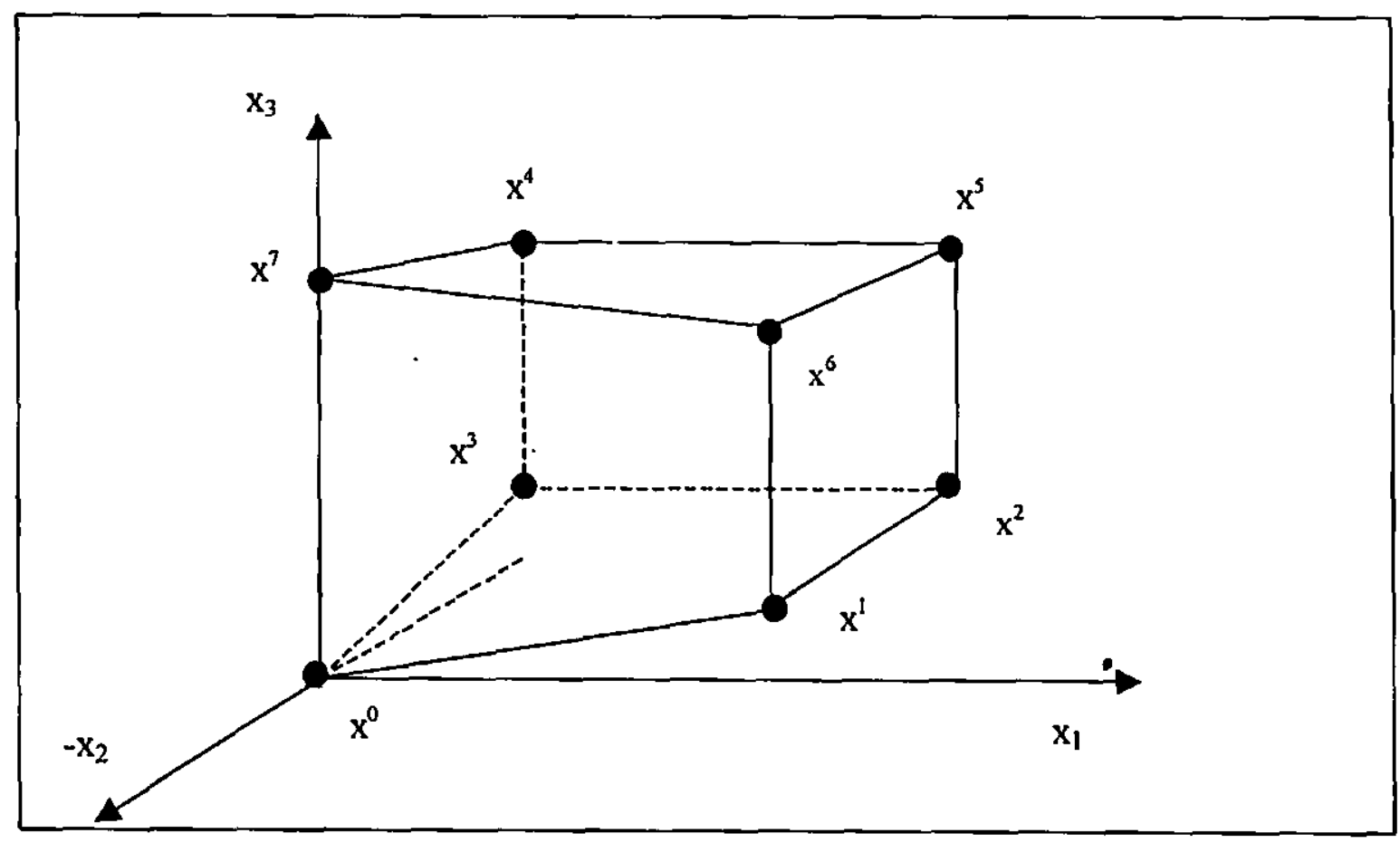

Figura 2 - Cubo deformado em $\mathrm{R}^{3}$

Assim temos que algoritmo simplex não pertence à classe de algoritmos com tempo polinomial.

\subsection{A Eficiência do Método Simplex}

Com relação ao bom desempenho que o método simplex apresenta na solução da grande maioria dos problemas, hoje identificados na prática, há classes de problemas não menos significativos onde o método simplex se mostra inoperante.

Como citado anteriormente, a eficiência do método é usualmente medida como sendo o número de iterações necessárias para solucionar um problema, expressada como uma função das dimensões do mesmo. Esta função, observada experimentalmente por pesquisadores da área, parecia uma função de grau baixo e talvez até linear. Obviamente o exemplo construído por Klee e Minty, apresentado na seção anterior, mostra que isto não é verdadeiro. Na maioria dos problemas reais, o número de iterações seria em média $\alpha m$, onde $m$ é o número de restrições e $\alpha$ um valor entre 2 e 3 (Shamir, 1987). Apresentaremos um estudo computacional para o problema de corte de peças, onde o número de iterações é da ordem de $\mathrm{m}^{2}$, reforçando o folclore simplex de que o número médio de iterações é um polinômio de grau baixo do número de restrições do problema. Para este assunto as considerações teóricas são feitas no final deste capítulo, enquanto que os resultados obtidos se encontram no capítulo $\mathrm{V}$. 
Variações do método simplex, mais tarde, também se mostraram ineficientes, do ponto de vista do estudo do pior caso, necessitando um número exponencial de iterações.

Portanto, a questão contínua aberta quanto à possibilidade da construção de um método do tipo simplex que seja polinomial, ou a prova definitiva de que é impossível construir um algoritmo do tipo simplex com complexidade polinomial. Segundo Shamir (1987), Terlaky e Zhang (1993) está é a questão aberta mais desafiante na teoria da otimização linear.

\subsection{Implementações do Método Simplex}

Conforme apresentado na seção 1.3 do capítulo I, os cálculos principais, por iteração, do método simplex consistem na resolução de três sistemas básicos:

$$
\begin{array}{lll}
1-\mathbf{x}_{\mathrm{B}}=\mathrm{B}^{-1} \mathbf{b} & \Leftrightarrow & \mathbf{B} \mathbf{x}_{\mathrm{B}}=\mathbf{b} \\
\text { 2- } \pi^{\mathrm{T}}=\mathbf{c}_{\mathrm{B}}^{\mathrm{T}} \mathbf{B}^{-1} & \Leftrightarrow & \mathbf{B}^{\mathrm{T}} \pi=\mathbf{c}_{\mathrm{B}} \\
\text { 3- } \mathbf{d}_{\mathrm{B}}=-\mathbf{B}^{-1} \mathbf{a}_{\mathrm{k}} & \Leftrightarrow & \mathbf{B} \mathbf{d}_{\mathrm{B}}=-\mathbf{a}_{\mathrm{k}}
\end{array}
$$

Obviamente os cálculos feitos para resolver um destes sistemas serão aproveitados na resolução dos demais, pois têm a mesma matriz dos coeficientes ou sua transposta. As primeiras implementações do método simplex optaram por trabalhar com $\mathbf{B}^{-1}$ (armazenada como produto de matrizes elementares, que será revisto a seguir), mas a decomposição de $\mathbf{B}$ em matrizes triangulares (decomposição LU) também seria natural. Além disso, procedimentos eficientes para recalcular, de uma iteração para outra, seja a inversa de $\mathbf{B}$ ou sua decomposição, se fazem necessários, pois apenas uma coluna de $\mathbf{B}$ é alterada. A seguir revisamos apenas dois desses procedimentos.

\subsubsection{Forma Produto da Inversa}

Suponha que numa iteração $t$, seja conhecido $\mathrm{B}^{(t)^{-1}}$, a inversa da matriz básica nesta iteração, $\mathbf{B}^{(t)}=\left[\mathbf{b}_{1}, \ldots, \mathbf{b}_{\mathrm{r}}, \ldots, \mathbf{b}_{\mathrm{m}}\right]$, onde $\mathbf{b}_{\mathfrak{i}}$ é a $i$-ésima coluna de $\mathbf{B}^{(\mathrm{t})}$.

A matriz básica da próxima iteração, $\mathbf{B}^{(t+1)}$ difere de $\mathbf{B}^{(t)}$ por apenas uma coluna (a $r$-ésima variável básica é trocada pela $k$-ésima variável não básica):

$$
\mathbf{B}^{(t+1)}=\left[\mathbf{b}_{1}, \ldots, \mathbf{a}_{\mathbf{k}}, \ldots, \mathbf{b}_{\mathrm{m}}\right] \text {, onde } \mathbf{a}_{\mathrm{k}} \text { é } k \text {-ésima coluna da matriz } \mathbf{N}
$$


Como na matriz básica mudou-se apenas uma coluna, é de se esperar que a inversa de $\mathbf{B}^{(t+1)}$ seja facilmente obtida a partir da inversa de $\mathbf{B}^{(t)}$.

De fato, multiplicando $\mathbf{B}^{(1)^{-1}} \mathbf{B}^{(1+1)}$ temos:

$$
\begin{aligned}
\mathbf{B}^{(1)^{-1}} \mathbf{B}^{(1+1)} & =\left[\mathbf{B}^{(1)^{-1}} \mathbf{b}_{1}, \ldots, \mathbf{B}^{(1)^{-1}} \mathbf{a}_{\mathbf{k}}, \ldots, \mathbf{B}^{(1)^{-1}} \mathbf{b}_{\mathrm{m}}\right] \\
& =\left[\begin{array}{cccccc}
1 & 0 & & y_{1} & & 0 \\
0 & 1 & & y_{2} & & 0 \\
& & \ddots & \vdots & & \\
\vdots & \vdots & \cdots & y_{r} & \cdots & \vdots \\
& & & \vdots & \ddots & \\
0 & 0 & & y_{m} & & 1
\end{array}\right]=\overline{\mathbf{I}}
\end{aligned}
$$

onde $\overline{\mathbf{I}}$ difere da matriz identidade apenas pela $k$-ésima coluna, uma vez que $\mathbf{B}^{(\mathrm{t})^{-1}} \mathbf{b}_{\mathbf{i}}=\mathrm{e}_{\mathbf{i}}$ : a $i$-ésima coluna da matriz identidade.

A inversa da matriz $\overline{\mathbf{I}}$, denotamos por $\mathbf{E}(\mathbf{E} \overline{\mathbf{I}}=\mathbf{I})$, é facilmente obtida:

$$
\mathbf{E}=\left[\begin{array}{cccccc}
1 & 0 & & -\frac{y_{1 k}}{y_{r k}} & & 0 \\
0 & 1 & & -\frac{y_{2 k}}{y_{r k}} & & 0 \\
& & \ddots & \vdots & & \\
\vdots & \vdots & \cdots & \frac{1}{y_{r k}} & \cdots & \vdots \\
& & & \vdots & \ddots & \\
0 & 0 & & -\frac{y_{m k}}{y_{r k}} & & 1
\end{array}\right]
$$

que corresponde a uma operação chamada "pivoteamento" no elemento $\mathrm{y}_{\mathrm{rk}}$ (isto é, a $k$-ésima coluna de $\overline{\mathrm{I}}$ é transformada por operações elementares na $r$-ésima coluna da matriz identidade.)

Com isto,

$$
B^{(1)^{-1}} B^{(1+1)}=\overline{\mathbf{I}} \Rightarrow \mathbf{E B}^{(1)^{-1}} B^{(1+1)}=\mathbf{E} \overline{\mathbf{I}}=\mathbf{I} \Rightarrow B^{(1+1)^{-1}}=\mathbf{E} B^{(1)^{-1}} .
$$

A equação acima nos diz que a inversa da nova base é obtida por estender para $\mathbf{B}^{()^{-1}}$ a operação de pivoteamento sobre $y_{k}$.

Supondo que $\mathbf{B}^{(0)}=\mathbf{I}$, e $\mathbf{E}_{1}, \mathbf{E}_{2}, \ldots, \mathbf{E}_{4}$ são as matrizes elementares obtidas nas atualizações das inversas nas iterações $1,2, \ldots, t$ podemos escrever: 


$$
\mathbf{B}^{()^{-1}}=\mathbf{E}_{1} \ldots \mathbf{E}_{2} \mathbf{E}_{1}
$$

Assim, a solução básica na iteração $t$ pode ser escrita como:

$$
x_{B}=B^{(1)^{-1}} b=E_{t}\left(E_{t-1} \ldots\left(E_{l} b\right)\right) .
$$

O vetor multiplicador simplex será:

$$
\left.\pi=\left(\left(\mathbf{c}_{\mathrm{B}}^{\mathrm{T}} \mathbf{E}_{\mathrm{t}}\right) \mathbf{E}_{\mathrm{t}-1}\right) \ldots \mathbf{E}_{2}\right) \mathbf{E}_{1} .
$$

O vetor das componentes básicas da direção simplex é calculado por:

$$
\mathbf{y}_{k}=\mathbf{B}^{-1} \mathbf{a}_{k}=\mathbf{E}_{\mathrm{t}}\left(\mathbf{E}_{\mathrm{t}-1} \ldots\left(\mathbf{E}_{\mathrm{j}} \mathbf{a}_{\mathrm{k}}\right)\right)
$$

É importante ressaltar que para a armazenagem da matriz $\mathbf{E}$, é necessário guardar somente $m+l$ números $\left(r, \mathrm{e}_{1}, \mathrm{e}_{2}, \ldots, \mathrm{e}_{\mathrm{m}}\right)$, onde $\mathrm{e}_{\mathrm{i}}$ são os elementos da matriz $\mathrm{E}$.

\subsubsection{Decomposição de Bartels e Golub (LU)}

Embora a forma produto da inversa seja conceitualmente importante e útil, é um tanto obsoleta, pois é baseada na técnica de eliminação de Gauss-Jordan para resolver os sistemas de equações. Apesar disto, muitos problemas práticos de porte médio são resolvidos eficientemente por esta forma. No capítulo $\mathrm{V}$ apresentamos algumas experiências computacionais. A técnica mais popularmente usada pela maioria dos pacotes de software para PL é a fatoração LU, que é baseada na estratégia mais eficiente de triangularização Gaussiana. Este procedimento é muito útil quando o problema é de grande porte e esparso. Além disso, pode-se recorrer à técnica de pivoteamento parcial, algo que faria $\left|-\mathbf{y}_{\mathrm{i}} / \mathbf{y}_{\mathrm{t}}\right| \leq 1$, evitando-se a propagação descontrolada dos erros de arredondamento. Observe que na técnica anterior o pivô $\mathbf{y}_{\mathrm{r}}$ está bem determinado, podendo ser pequeno e produzindo $|\mathrm{e}| \mathrm{i} \mid>1$, fazendo com que os inevitáveis erros de arredondamento se propaguem rapidamente.

Bartels e Golub (1969) propuseram uma técnica para atualizar a decomposição LU da matriz básica, permitindo-se um pivoteamento parcial, revisado a seguir. 
Suponha que a matriz $\mathbf{B}$ (permutada) seja decomposta em $\mathbf{P B}=\mathbf{L U}$, onde $\mathbf{P}$ é matriz de permutação, indicando as linhas trocadas durante o processo de eliminação de Gauss, $\mathbf{U}$ é uma matriz triangular superior e $\mathbf{L}$ é uma matriz triangular inferior, onde os elementos da diagonal desta última são iguais a 1. Esta permutação é feita de forma que o elemento pivô seja o maior elemento em módulo da coluna. Isto faz com que os elementos de $\mathbf{L}$ sejam menores ou iguais a 1 , proporcionando assim uma menor propagação nos erros de arredondamento na resolução dos sistemas.

Dado o sistema $B v=q$, este será resolvido encontrando-se as soluções dos sistemas triangulares descritos abaixo:

$$
\begin{gathered}
\mathbf{B} v=q \Leftrightarrow \mathbf{P B} v=\mathbf{P} q \\
\Leftrightarrow \mathbf{L U} v=\mathbf{P} q .
\end{gathered}
$$

Assim temos dois sistemas triangulares:

$$
\mathbf{L} t=\mathbf{P} \boldsymbol{q} \quad \mathrm{e} \quad \mathrm{U} \boldsymbol{v}=\boldsymbol{t}
$$

Considere que a base inicial seja $\mathbf{B}^{(0)}=\left[\mathbf{b}_{1}, \mathbf{b}_{2}, \ldots, \mathbf{b}_{\mathrm{r}_{1}}, \ldots, \mathbf{b}_{\mathrm{m}}\right]$ e que $r_{l}$-ésima coluna básica deixa a base e $k_{l}$-ésima coluna de $\mathbf{N}$ entra na base, na primeira iteração. A nova matriz básica é organizada da seguinte forma:

$\mathbf{B}^{(1)}=\left[\mathbf{b}_{1}, \ldots, \mathbf{b}_{\mathrm{r}_{1}-1}, \mathbf{b}_{\mathrm{r}_{1}+1}, \ldots, \mathbf{b}_{\mathbf{m}}, \mathbf{a}_{\mathrm{k}_{1}}\right]$, onde $\mathbf{a}_{\mathrm{k}_{1}}$ é a $k_{l}$-ésima coluna de $\mathbf{N}$. A transição da decomposição de $\mathrm{B}^{(0)}$ para a decomposição de $\mathbf{B}^{(1)}$, é particularmente fácil como também estável, como veremos a seguir:

$$
\begin{aligned}
\mathbf{L}^{-1} \mathbf{P} \mathbf{B}^{(1)} & =\left[\mathbf{L}^{-1} \mathbf{P} \mathbf{b}_{1}, \ldots, \mathbf{L}^{-1} \mathbf{P} \mathbf{b}_{r_{1}-1}, \mathbf{L}^{-1} \mathbf{P} \mathbf{b}_{r_{1}+1}, \ldots, \mathbf{L}^{-1} \mathbf{P} \mathbf{b}_{\mathfrak{m}}, \mathbf{L}^{-1} \mathbf{P} \mathbf{a}_{k_{1}}\right] \\
& =\left[\mathbf{u}_{1}, \ldots, \mathbf{u}_{r_{1}-1}, \mathbf{u}_{r_{1}+1}, \ldots, \mathbf{u}_{\mathfrak{m}}, \mathbf{L}^{-1} \mathbf{P} \mathbf{a}_{\mathbf{k}_{1}}\right] \\
& =\mathbf{H}^{(1)}
\end{aligned}
$$

onde $\mathbf{u}_{\mathrm{i}}$ são as colunas da matriz U. A matriz $\mathbf{H}^{(1)}$ é da forma:

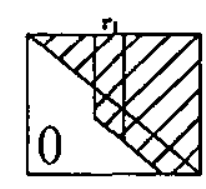


Observe que a matriz coluna $\mathbf{L}^{-1} \mathbf{P} \mathbf{a}_{\mathrm{k}_{1}}$ já foi obtida, quando do cálculo da direção simplex, no algoritmo simplex, antes da atualização da base (PBy $=-\mathbf{P} \mathbf{a}_{\mathrm{k}_{1}} \Rightarrow \mathbf{L U y}=-\mathbf{P} \mathbf{a}_{\mathrm{k}_{1}} \Rightarrow$ $\left.\Rightarrow t=-\mathbf{L}^{-1} \mathbf{P} \mathbf{a}_{\mathbf{k}_{1}}\right)$. Assim $\mathbf{H}^{(1)}$ pode ser construída sem qualquer esforço computacional extra.

Podemos reduzir $\mathbf{H}^{(1)}$ a uma matriz triangular superior $\mathbf{U}^{(1)}$ usando operações elementares para zerar os elementos da subdiagonal da coluna $r_{l}$ até a coluna $m$. A seleção do pivô é realizada em cada passo da eliminação, podendo haver ou não troca entre duas linhas adjacentes. Assim $\mathbf{U}^{(1)}$ é obtida da forma $\mathbf{H}^{(1)}$ aplicando uma seqüência de operações elementares a esquerda de $\mathbf{H}^{(1)}$, ou seja,

$$
\mathbf{U}^{(1)}=\Gamma_{m-1}^{(1)} \mathbf{P}_{m-1}^{(1)} \ldots \Gamma_{r_{1}}^{(1)} \mathbf{P}_{r_{1}}^{(1)} \mathbf{H}^{(1)}
$$

onde cada $\Gamma_{i}^{(1)}$ tem a forma:

$i \quad i+1$

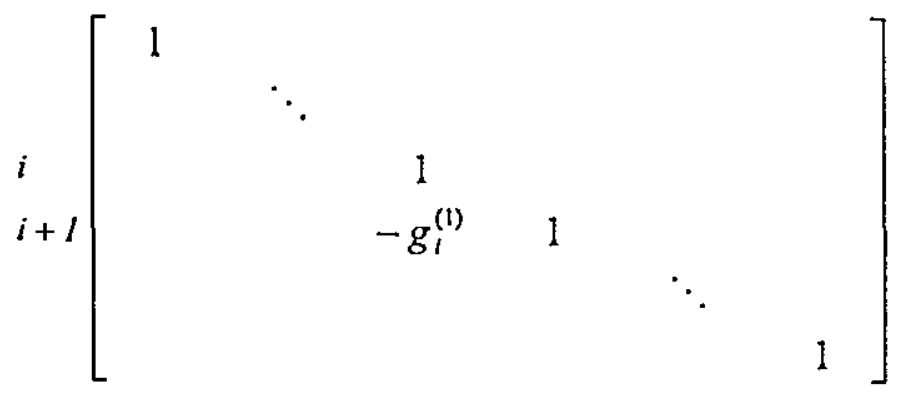

e cada $\mathbf{P}_{i}^{(1)}$ é a matriz identidade ou uma permutação desta com a $i$-ésima e a $i+1$-ésima linhas trocadas de posição. A escolha é tal que $\left|g_{i}^{(1)}\right| \leq 1$ (onde $g_{i}^{(1)}$ é o multiplicador).

Portanto, substituindo (2.3) em (2.2) segue:

$$
\mathbf{P B}^{(1)}=\mathbf{L} \mathbf{P}_{\mathrm{r}_{1}}^{(1)} \boldsymbol{\Gamma}_{\tau_{1}}^{(1)^{-1}} \ldots \mathbf{P}_{m-1}^{(1)} \Gamma_{m-1}^{(1)-1} \mathbf{U}^{(1)}
$$

observando que $\mathbf{P}_{i}^{(1)^{-1}}=\mathbf{P}_{i}^{(1)}$.

Se outra iteração do método simplex é realizada, a matriz base resultante $\mathbf{B}^{(2)}$ pode ser decomposta a partir da decomposição de $\mathbf{B}^{(1)}$, da mesma maneira que $\mathbf{B}^{(i)}$ foi decomposta de $\mathbf{B}^{(0)}$. Por conveniência de notação, sejam 


$$
\mathbf{C}^{(i)}=\mathbf{P}_{r_{1}}^{(1)} \Gamma_{r_{1}}^{(1)^{-1}} \ldots P_{m-1}^{(1)} \Gamma_{m-1}^{(1)}
$$

e

$$
\mathbf{G}^{(t)}=\mathbf{C}^{(\mathrm{i})} \ldots \mathbf{C}^{(\mathrm{t})}
$$

A decomposição da matriz $\mathrm{B}^{(\mathrm{t})}$ na iteração $t$ é dada por:

$$
\begin{aligned}
\mathbf{P B}^{(t)} & =\mathbf{L}\left[\mathbf{P}_{\mathrm{r}_{1}}^{(1)} \Gamma_{\mathrm{r}_{1}}^{(1)^{-1}} \ldots \mathbf{P}_{m-1}^{(1)} \Gamma_{m-1}^{(1)-1}\right] \ldots\left[\mathbf{P}_{\mathrm{r}_{1}}^{(t)} \Gamma_{\mathrm{r}_{1}}^{()^{-1}} \ldots \mathbf{P}_{m-1}^{(t)} \Gamma_{m-1}^{(t)-1}\right] \mathbf{U}^{(t)} \\
& =\mathbf{L} \mathbf{C}^{(\mathbf{i})} \ldots \mathbf{C}^{(t)} \mathbf{U}^{(t)} \\
& =\mathbf{L} \mathbf{G}^{(t)} \mathbf{U}^{(t)}
\end{aligned}
$$

onde cada matriz envolvida na decomposição é calculada de forma análoga à primeira iteração.

Cada sistema linear dado

$$
\mathrm{PB}^{(\mathrm{t})} v=\mathbf{P} q
$$

é resolvido achando a solução do triangular inferior

$$
\mathbf{L} t=\mathbf{P} \boldsymbol{q}, \text { onde } \boldsymbol{t}=\mathbf{G}^{(t)} \mathrm{U}^{(t)} \boldsymbol{v}
$$

e fazendo $w=\mathrm{U}^{(t)} v \Rightarrow w=\mathbf{G}^{\left(t^{-1}\right.} t$

Logo,

$$
w=\left[\left[\Gamma_{m-1}^{(1)} \mathbf{P}_{\mathrm{m}-1}^{(t)} \ldots \Gamma_{\mathrm{r}_{\mathrm{t}}}^{(1)} \mathbf{P}_{\mathrm{r}_{\mathrm{t}}}^{(t)}\right] \ldots\left[\Gamma_{\mathrm{m}-1}^{(1)} \mathbf{P}_{\mathrm{m}-1}^{(1)} \ldots \Gamma_{\mathrm{r}_{\mathrm{l}}}^{(1)} \mathbf{P}_{\mathrm{r}_{\mathrm{f}}}^{(1)}\right]\right] t
$$

e finalmente resolvendo o sistema triangular superior:

$$
\mathrm{U}^{(t)} v=w .
$$

O procedimento para resolver $\mathrm{B}^{(1)^{\mathrm{T}}} \boldsymbol{v}=\boldsymbol{q}$ é análogo.

As implementações do método dual simplex consideradas no capítulo $\mathrm{V}$, utilizam a técnica de Bartels Golub.

\subsection{Programação Linear Aplicada ao Problema de Corte}

Problemas de corte de estoque são essenciais no planejamento da produção em várias indústrias, tais como indústrias de papel, aço, têxtil, etc. Estes problemas consistem em produzir um conjunto de itens obtidos a partir da cortagem de peças em estoque, com o objetivo de atender a uma demanda, otimizando uma determinada função objetivo. Estes problemas podem ser formulados como problemas de Otimização Linear Inteira. Gilmory e Gomory (1961) relaxaram a condição de integralidade e propuseram um método eficiente de geração de colunas para a resolução do problema linear resultante. Para se obter a solução 
inteira do problema, várias heurísticas são desenvolvidas para o obtenção desta s' partir da solução aproximada. Para um estudo aprofundado sobre o assunto, veja Pint

A formulação do problema de corte de estoque inteiro bidimensional é da seguinte forma:

$$
\begin{aligned}
& \text { Minimizar } \mathrm{Z}(\mathbf{x})=\sum_{\mathrm{j}=1}^{\mathrm{n}} \mathrm{x}_{\mathrm{j}} \\
& \text { Sujeito a: } \sum_{\mathrm{j}=1}^{\mathrm{n}} \mathrm{a}_{\mathrm{ij}} \mathrm{x}_{\mathrm{j}} \geq \mathrm{d}_{\mathrm{i}}, i=1, \ldots, m \\
& x_{j} \geq 0 \text { e inteiro, } j=1, \ldots n
\end{aligned}
$$

onde cada coluna $\left(a_{1 j} a_{2 j} \ldots a_{m j}\right)$ fornece o número de itens obtidos no $j$-ésimo padrão de corte, a variável $x_{j}$ indica o número de repetições do padrão de corte e $d_{\mathrm{i}}$ a demanda do item $i$.

A exigência de integralidade sobre as variáveis $x_{j}$ torna o problema difícil de ser resolvido ao considerarmos problemas grandes, e em problemas práticos, $m$ (o número de tipos de itens) é da ordem de algumas dezenas, enquanto que $n$ pode ser da ordem de vários milhões, inviabilizando a resolução direta do problema. Com isto, a relaxação por programação linear, a utilização do processo de geração de colunas, bem como os estudos de métodos heurísticos, são importantes na resolução do problema acima. Ressalta-se porém, que o estudo de métodos heurísticos não serão abordados aqui, já que o propósito é analisar o comportamento computacional do método simplex, quando aplicado à relaxação do problema de corte de estoque.

\subsubsection{Geração de Colunas}

O processo de geração de colunas é adequado ao método simplex para determinar a nova coluna que deverá entrar na base melhorando a solução. A escolha da coluna a entrar na base pode ser bastante complexa devido ao grande número de colunas a serem investigadas. Este processo foi proposto por Gilmore e Gomory (1961) e consiste em encontrar a coluna $j$, com o menor custo relativo, dado por: $c_{j}-\pi^{\top} \mathbf{a}_{j}$. Como $c_{j}=1$, o problema resulta em:

$$
\min c_{j}-\pi^{T} a_{j}=\min 1-\pi^{T} a_{j}=\min 1-\pi_{1}^{T} a_{i j}-\pi{ }_{2}^{T} a_{2 j}-\ldots-\pi{ }_{m}^{T} a_{m j} .
$$


Observe que a coluna $\left(a_{1 j} a_{2 j} \ldots a_{m j}\right)^{T}$ corresponde a um padrão de corte e não precisa estar disponível.

Um padrão de corte pode ter apenas uma dimensão relevante (corte de barras, bobinas), duas (placas de madeira, vidro), etc.

Neste trabalho utilizamos o estudo de Pinto (1999) para o caso de problemas de corte de placas, digamos $L \times W$. Há várias restrições do processo de cortagem. Aqui consideramos padrões guilhotinados em 2-estágios que definimos a seguir.

Definição 2.1: Um corte é dito guilhotinado se aplicado a um retângulo produzir dois novos retângulos. Um padrão de corte é do tipo guilhotinado se for obtido através de cortes guilhotinados sucessivos.

Os cortes guilhotinados são classificados em estagiados e não-estagiados. Um estágio é definido através da produção de um certo número de cortes guilhotinados numa mesma direção sobre a placa ou sobre partes dela, resultantes do estágio anterior. Os estágios sucessivos alternam a direção dos cortes, isto é, os cortes produzidos em um estágio subsequente são ortogonais ao corte produzido no estágio antecedente.

Definição 2.2: Um corte é dito 2-estágios quando apenas uma mudança no sentido dos cortes guilhotinados é permitida.

Um padrão 2-estágios é obtido em duas etapas:

Etapa 1: Considerando que o primeiro corte feito na placa seja horizontal, primeiramente deve-se definir faixas de dimensão $L \times w_{j}$ e descrever padrões de corte para cada uma delas. Neste caso, temos vários problemas unidimensionais.

Etapa 2: Determinar quantas vezes cada faixa, com seu respectivo padrão, será utilizada na placa de dimensão $L \times W$.

Considerando uma coluna genérica do modelo (2.4) por $\left(\mathrm{y}_{1} \mathrm{y}_{2} \ldots \mathrm{y}_{\mathrm{m}}\right)$, o problema (2.5) pode ser escrito por:

$\operatorname{Maximizar} \sum_{\mathbf{i}} \pi_{\mathrm{i}} \mathrm{y}_{\mathrm{i}}$

Sujeito a: $\left(\mathrm{y}_{1}, \mathrm{y}_{2}, \ldots, \mathrm{y}_{\mathrm{m}}\right)^{\mathrm{T}}$ - corresponda a um padrão de corte para a placa $\mathrm{L} \times \mathrm{W}$ 
onde $\mathbf{a}=\left(\mathrm{y}_{1}, \mathrm{y}_{2}, \ldots, \mathrm{y}_{\mathrm{m}}\right)^{\mathrm{T}}$ significa que $\mathrm{y}_{\mathrm{i}}$ itens do tipo $i$ foram produzidos no padrão de corte correspondente.

A solução dỏ problema (2.6) fornece a coluna com o menor custo relativo $\left(1-\sum_{i} \pi_{i} y_{i}\right)$ para o problema (2.4). Se este custo relativo for não negativo, então a solução básica é ótima.

\subsubsection{Método Simplex com Geração de Colunas Aplicado ao Problema de Corte}

Para a obtenção de uma matriz básica inicial, no caso do problema de corte de estoque, consideramos os padrões de corte homogêneo, ou seja, de modo que cada padrão produza uma coluna do tipo: $\left(0, \ldots, \mathrm{a}_{\mathrm{i}} ; \ldots, 0\right)^{\mathrm{T}}$ onde $\left.\mathrm{a}_{\mathrm{i}}=\left\lfloor\frac{L}{l_{\mathrm{i}}}\right\rfloor \frac{W}{w_{i}}\right\rfloor . \quad$ Apresentamos em seguida o algoritmo do método primal simplex aplicado ao problema de corte de estoque bidimensional, para o qual estamos interessados no resultado computacional que será descrito no capítulo $\mathrm{V}$ :

Algoritmo Primal Simplex com Geração de Colunas:

\section{Fase I:}

Gere a matriz básica inicial B (diagonal), utilizando as soluções homogêneas, da seguinte forma:

$$
B=\left(\begin{array}{cccccc}
\left\lfloor\frac{L}{l_{l}}\right\rfloor \times\left\lfloor\frac{W}{w_{l}}\right\rfloor & 0 & 0 & 0 & \ldots & 0 \\
0 & \left\lfloor\frac{L}{l_{2}}\right\rfloor \times\left\lfloor\frac{W}{w_{2}}\right\rfloor & 0 & 0 & \ldots & 0 \\
\vdots & \vdots & \vdots & \ddots & \vdots \\
0 & 0 & 0 & 0 & \ldots & \left.\frac{L}{l_{m}}\right\rfloor \times\left\lfloor\frac{W}{w_{m}}\right\rfloor
\end{array}\right)
$$


Faça $P A \dot{R} E=F A L S O, I T=0$

\{A variável lógica PARE terá valor Falso até que a condição de otimalidade seja verificada e $I T$ indica o número da iteração atual\}

Fase II:

Enquanto $N \tilde{A} O P A R E$, faça:

\{Início da Iteração Simplex\}

1. Determine a solução básica primal factível: $\mathbf{B x}=\mathbf{d}$. i

2. Teste a otimalidade:

2.1. Determine a solução básica dual: $\mathbf{B}^{\mathrm{T}} \pi=\mathbf{e}$. Onde $\mathbf{e}=(1, \ldots, 1)^{\mathrm{T}}$

2.2. Resolva o Problema (2.6) \{Geração de colunas\}

2.3. Se $1-\sum_{\mathrm{i}=1}^{\mathrm{m}} \pi_{\mathrm{i}} \mathrm{y}_{\mathrm{i}} \geq 0$ então $P A R E=V E R D A D E$ \{solução atual é ótima, obtida em $I T$ iterações\}

3. Senão determine as coordenadas básicas da direção simplex (ds): $\mathbf{B}(\mathbf{d s})=-\mathbf{y}$

4. Determine o tamanho do passo

Encontre $r$ tal que:

$$
\frac{-x_{r}}{d s_{r}}=\min \left\{\frac{-x_{i}}{d s_{i}} \mid d s_{i}<0\right\} .
$$

5. Atualize a base, colocando a coluna y na posição da $r$-ésima coluna da matriz $\mathbf{B}$. Faça: $I T=I T+1 . \quad$ (Fim da Iteração Simplex\} 


\section{Capítulo III}

\section{Variantes do Método Simplex}

\subsection{Introdução}

Como foi dito no capítulo I, a programação linear tem sido uma das áreas mais dinâmicas de matemática aplicada nas últimas décadas. Embora recentemente muitas novas abordagens tenham sido sugeridas para resolver um problema de programação linear, como método de pontos interiores, o tradicional método simplex de Dantzig é ainda o algoritmo mais eficiente para a maioria dos problemas práticos.

O método simplex é muito flexível, no sentido que existem várias maneiras de selecionar as variáveis que entra e que sai da base. Portanto, não é uma surpresa que muitos variantes simplex tenham sido desenvolvidos nos últimos anos.

Como o número de métodos desenvolvidos para problemas de otimização linear é vasto, optamos por apresentar apenas informações gerais de alguns destes métodos recentes que encontramos na literatura.(Terlaky e Zhang, 1993).

De acordo com Terlaky e Zhang (1993), a regra recursiva de Bland é o primeiro "esquema" geral de recursividade do método simplex preservando factibilidade, ao passo que o método de Jensen (1985) generaliza essa recursão a bases infactíveis.

Os métodos criss-cross deram uma resposta positiva para a seguinte questão: Existe um método finito (talvez infactível) com abordagem do tipo simplex, o qual resolve um . 
programa linear em uma fase? O primeiro método do tipo criss-cross foi designado por Zionts (1969). Terlaky (1987) apresenta um variante.

Métodos criss-cross podem ser inicializado com qualquer solução básica, na busca da solução ótima ou identificação de infactibilidade. Infelizmente para o método desenvolvido por Zionts, não está claro, em geral, se este é finito, enquanto o de Terlaky (1987) é um método finito. Zhang (1999) apresenta um novo variante finito do método criss-cross para programação linear.

\subsection{Regras combinatórias e finitas}

Nesta seção revisaremos as duas regras de pivoteamentø de Bland, as quais preocupam-se somente com as infactibilidades primal e dual. Terlaky e Zhang (1993) classificam esses tipos de regras como regras de pivoteamento combinatório. Um exemplo de uma regra de pivoteamento não combinatório é dado pela conhecida regra de Dantzig, a qual sempre seleciona a variável com o custo reduzido mais negativo. Ela não envolve somente o sinal das variáveis, mas também a magnitude das mesmas.

Segundo Terlaky e Zhang (1993), a maioria das regras combinatórias são originalmente desenvolvidas no contexto de uma forma abstrata de programação linear, isto é, chamada de programação matroid. Para um estudo da teoria de programação matroid que foi estabelecida por Bland (1977), Folkman e Lawrence (1978) referência-se o trabalho de Fukuda (1982).

Vamos apresentar a seguir, as duas regras de Bland (1977). Essas regras partem de uma base factível e mantém a factibilidade primal. A primeira regra de Bland é conhecida como "regra do menor índice", é muita citada por evitar ciclagem do método simplex, e devido a sua simplicidade. Entretanto, segundo Avis e Chavatal (1978) o desempenho computacional do método simplex, sob esta regra, decai.

\subsubsection{Regra do Menor Índice de Bland}

Passo1: (Escolha da variável de entrada) Escolha a variável não básica que tem o menor índice entre as variáveis com custos reduzidos negativos, ou seja, selecione uma variável não básica, digamos $\mathrm{x}_{\mathrm{k}}$, para entrar na base tal que:

$$
k=\operatorname{minímo}\left\{j \mid \mathrm{c}_{\mathrm{j}}-\pi^{\mathrm{T}} \mathbf{a}_{\mathrm{j}}<0, j \in \mathrm{N}\right\}
$$


Se não existe tal variável a solução atual é ótima.

Passo 2: (Escolha da variável de saída) Escolha uma variável para sair da base, digamos $\mathrm{x}_{\mathrm{r}}$, tal que após a mudança a base permaneça factível e o índice desta variável seja o menor entre todos aqueles com tal possibilidade. Isto é:

$$
r=\operatorname{mínimo}\left\{t \mid \mathrm{y}_{\mathrm{tk}}<0 \text { e } \frac{\mathrm{x}_{\mathrm{B}_{\mathrm{t}}}^{0}}{\mathrm{y}_{\mathrm{tk}}}=\min \left\{\frac{\mathrm{x}_{\mathrm{B}_{\mathrm{i}}}^{0}}{\mathrm{y}_{\mathrm{ik}}} \mid \mathrm{y}_{\mathrm{ik}}<0\right\} i \in \mathrm{B}\right\}
$$

Observe que o procedimento para manter a factibilidade da base é o teste da menor razão, que se encontra no capítulo $I$.

A segunda regra de Bland é bem menos conhecida. De fato, ela propõe um procedimento recursivo. A idéia deste procedimento é baseado na observação elementar que uma base primal factívęl será ótima se todas as variáveis duais infactíveis fossem apagadas do problema. Essa regra para iniciar necessita-se de uma solução primal factível.

\subsubsection{Regra Recursiva de Bland}

Considere o seguinte problema $(\mathrm{P})$ de programação linear:

$$
\begin{aligned}
& \text { Minimizar } Z(\mathbf{x})=\mathbf{c}^{\mathrm{T}} \mathbf{x} \\
& \text { Sujeito a: } \quad \mathbf{A x}=\mathbf{b} \\
& \qquad \mathrm{x}_{\mathrm{j}} \geq 0 \forall j \in \mathrm{E}=\{1, \ldots, n\}
\end{aligned}
$$

onde $\mathbf{A}$ tem dimensão $m \times n$ e posto $(\mathbf{A})=m$. Suponha que $(\mathbf{B}, \mathbf{N})$ seja uma partição básica factível, e $\overline{\mathbf{x}}=\left(\mathbf{B}^{-1} \mathbf{b}, 0\right)$ a solução associada para o problema $(P)$.

Se para algum $S \subseteq E$ e $k \in E l S$ e tem-se $S \cap B=\varnothing, c_{k}-\pi a_{k}<0$ e $c_{j}-\pi a_{j} \geq 0$ para todo $j \in \mathrm{E} \backslash(\mathrm{S} \cup\{\mathrm{k}\})$, então diz que a partição $(\mathrm{B}, \mathrm{N})$ é redutivel com relação ao conjunto $\mathrm{S}$. Lembrando que B é o conjunto de índices básicos. Bland (1977) utiliza a seguinte proposição como ponto chave de sua regra. 
Proposição 3.1. Se $(B, N)$ é redutível com relação a $S$, então para qualquer $\hat{\mathbf{x}}$ satisfazendo $\mathbf{A} \hat{\mathbf{x}}=\mathbf{b}, \hat{\mathbf{x}}_{j}=0, \forall j \in \mathrm{S}, \hat{\mathbf{x}}_{j} \geq 0 \forall j \in E \backslash(\mathrm{S} \cup\{\mathrm{k}\})$, e $\mathbf{c}^{\mathrm{T}} \hat{\mathbf{x}} \geq Z(\overline{\mathbf{x}})$, segue que $\hat{\mathbf{x}}_{\mathrm{k}} \geq 0$.

Suponha que $(\mathbf{B}, \mathbf{N})$ seja redutível com relação a $S$, entãó deseja-se resolver o subproblema $\left(\mathrm{P}^{\prime}\right)$ obtido de $(\mathrm{P})$ eliminando-se as variáveis cujos os índices estão em $\mathrm{S}$, isto é, fazendo $\mathrm{x}_{\mathrm{j}}=0$ para todo $\forall j \in \mathrm{S}$. A partição $(\mathrm{B}, \mathrm{N})$ representa uma partição básica factível para $\left(\mathrm{P}^{\prime}\right)$. Escolhendo-se a variável $\mathrm{x}_{\mathrm{k}}$ para entrar na base, ou resolve ( $\mathrm{P}^{\prime}$ ) ou produz uma nova partição básica $\left(\mathbf{B}^{\prime}, \mathbf{N}^{`}\right)$, na qual $\mathrm{x}_{\mathrm{k}}$ é uma variável básica e $\mathrm{c}_{\mathrm{j}}-\pi^{`} \mathbf{a}_{\mathrm{j}}<0$ para algum $j \in \mathrm{ElS}$. No último caso, segundo Bland (1977), da proposição 3.1, pode-se ignorar $x_{k}$ como candidata a sair da base durante toda os passos subsequentes até ( $\mathrm{P}^{\prime}$ ) ser resolvido. Portanto, isto garante que a partição $(\mathbf{B}, \mathbf{N})$ não se repetirá durante a resolução do problema em questão.

Assim a variável básica $\mathrm{x}_{\mathrm{k}}$ é desnecessária; com isso o número de restrições ativas é reduzido a $(m-1)$. Portanto, resolve-se um problema reduzido que é equivalente ao problema $\left(\mathrm{P}^{\prime}\right)$, e se existir uma solução ótima para ele restaure a variável $\mathrm{x}_{\mathrm{k}}$.

$O$ autor considera o seguinte problema $(Q)$, para mostrar como usar a idéia acima para construir um método simplex finito:

Problema (Q):

$$
\begin{aligned}
& \text { Minimizar } Z \begin{array}{l}
Z(\mathbf{x})=\mathbf{c}^{\top} \mathbf{x} \\
\text { Sujeito a: } \quad \mathbf{A x}=\mathbf{b} \\
\mathrm{x}_{\mathrm{j}} \geq 0 \forall j \in \mathrm{E} \backslash \mathrm{S} \\
\mathrm{x}_{\mathrm{j}}=0 \forall j \in \mathrm{S}
\end{array}
\end{aligned}
$$

onde $\mathrm{S} \subseteq \mathrm{E}$.

Suponha que $\left(\mathbf{B}^{0}, \mathbf{N}^{0}\right)$ seja uma partição factível para $(Q)$ e a solução básica factível correspondente é $\mathrm{x}^{0}=\left(\mathrm{x}_{1}^{0}, \ldots, \mathrm{x}_{\mathrm{n}}^{0}\right)$ com $\mathrm{B}^{0} \cap \mathrm{S}=\varnothing$, onde $\mathrm{B}^{0}$ é o conjunto de índices das variáveis básicas. Considere o seguinte procedimento para resolver (Q).

\section{Procedimento Recursivo de Bland}

Passo 0 - Inicialmente $i=0$.

Passo $1-$ Seja $D^{i}=\left\{j \in\right.$ ElS : $\left.\left(c_{j}-\pi^{i} a_{j}\right)<0\right\} .\left(\pi^{i}:\right.$ multiplicador simplex associado à partição $\left(\mathbf{B}^{\mathbf{i}}, \mathbf{N}^{\mathbf{i}}\right)$ )

Se $D^{i}=\varnothing$, então $x^{i}$ é uma solução ótima de (Q). 
Caso contrário, escolha algum $k \in \mathrm{D}^{i}$ e faça $\mathrm{S}^{i+1}=\mathrm{S} \cup \mathrm{D} \backslash\{k\}$.

Passo 2 - Resolva o subproblema $\left(Q^{i+1}\right)$ obtido de (Q) substituindo $S$ por $S^{i+1}$ em (3.2). Como $\mathrm{B}^{\mathrm{i} \cap} \mathrm{S}^{\mathrm{i}+1}=\varnothing, \mathrm{x}^{\mathrm{i}}$ é uma solução básica factível de $\left(\mathrm{Q}^{\mathrm{i+1}}\right)$.

Se $\left(Q^{i+l}\right)$ for ilimitado, então $(Q)$ é ilimitado. Senão, sejam $\left(B^{i+1}, N^{i+l}\right)$ uma partição básica ótima para $\left(Q^{i+1}\right)$ tal que $B^{i+1} \cap S^{i+1}=\varnothing$ e $x^{i+1}$ é a solução ótima.

Faça $i=i+1$ ęá ao passo 1 .

Observação 3.1. Como $\mathrm{S}^{\mathrm{i}+1} \subset \mathrm{S}^{\mathrm{i}}$, para $i \geq 1$, o procedimento acima resolve (Q) após resolver finitamente muitos subproblemas, digamos $\left(Q^{1}\right), \ldots,\left(Q^{\prime}\right)$.

Observação 3.2. A partição $\left(\mathbf{B}^{\mathrm{i}}, \mathrm{N}^{\prime}\right)$ é redutível com relação a $\mathrm{S}^{\mathrm{i}+1}, i=0, \ldots, l-1$. Daí a iteração simplex, pode-se resolver $\left(Q^{i+1}\right)$, ou reduzir $\left(Q^{i+1}\right)$ em um problema linear equivalente com uma restrição a menos.

Dado qualquer problema linear (P) na forma (3.1), pode-se aplicar o procedimento acima recursivamente para resolvê-lo $(\mathrm{P})$ a partir de qualquer solução factível. Inicialmente seja $(\mathrm{Q})=(\mathrm{P})$ no procedimento recursivo de Bland com $\mathrm{S}=\varnothing$; desta maneira cria-se uma sequência $\left(\mathrm{P}^{\mathrm{l}}\right), \ldots,\left(\mathrm{P}^{l}\right)$ de subproblemas, cada um na forma (3.2).

Seja o subconjunto $S$ para o subproblema $\left(\mathrm{P}^{\mathrm{i}}\right)$ denotado por $\mathrm{S}^{\mathrm{i}}$. Quando o subproblema $\left(\mathrm{P}^{\mathrm{i}}\right)$ é criado, tem-se a partição básica factível para $\left(\mathrm{P}^{\mathrm{i}}\right)$ que é redutível com relação a $\mathrm{S}^{i+1}$. Uma simples iteração resolve $\left(\mathrm{P}^{\mathrm{i}}\right)$, ou reduz ele a um subproblema $\left(\hat{\mathrm{P}}^{\mathrm{i}}\right)$ equivalente com dimensão $(m-1) \times(n-1)$. Na última situação, faça $(\mathrm{Q})=\left(\hat{\mathrm{P}}^{\mathrm{i}}\right)$ e continue o processo.

As observações 3.1 e 3.2 podem ser aplicadas (recursivamente) para mostrar que tal aplicação recursiva do procedimento resolverá qualquer problema linear (P) após um número finito de passos. Observe que a recursão pode efetuar reduções sucessivas de modo que o número de linhas no problema reduzido pode chegar até somente uma restrição.

Note também, que a aplicação recursiva do procedimento descrito acima é um método do tipo simplex, apesar de que algumas iterações são realizadas em um problema reduzido.

Para deixar como o método trabalha, considere o seguinte problema de programação linear que já se encontra na forma padrão. Para resolvê-lo considere inicialmente $S=\varnothing$ : 
Minimizar $Z(x 1, x 2)=-x 1-2 x 2$

$$
\text { Sujeito a: } \begin{array}{rlr}
\mathrm{x} 1+\mathrm{x} 2+\mathrm{x} 3 & =6 \\
\mathrm{x} 1-\mathrm{x} 2 & +\mathrm{x} 4 \quad=4 \\
-\mathrm{x} 1+\mathrm{x} 2 & +\mathrm{x} 5=4 \\
\mathrm{xj} \geq 0 \forall j \in \mathrm{E}=\{1, \ldots, 5\}
\end{array}
$$

Como $S=\varnothing$, inicialmente nenhuma variável está fixa em zero, isto é $(Q)=(P)$. Observe que para cada problema $\left(\mathrm{P}^{\mathrm{i}}\right)$ o conjunto $\mathrm{S}^{0}=\varnothing$. Então usando o quadro simplex

\begin{tabular}{|c|c|c|c|c|c|c|}
\hline & $\mathrm{X} 1$ & $x 2$ & $x 3$ & $\times 4$ & $\times 5$ & \\
\hline Z & 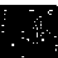 & & 8,40 & 805 & 8030 & 0 \\
\hline$x 3$ & 1 & 1 & 1 & 0 & 0 & 6 \\
\hline$\times 4$ & 1 & -1 & 0 & 1 & 0 & 4 \\
\hline$x 5$ & -1 & 1 & 0 & 0. & 1 & 4 \\
\hline
\end{tabular}
(apêndice), temos:

$\underline{i t=0}$

Tableau 0 - correspondente ao problema (P)

\begin{tabular}{|c|c|c|c|c|c|c|}
\hline & $\mathrm{X} 1$ & $\times 2$ & $x 3$ & $\times 4$ & $x 5$ & \\
\hline$Z$ & $c^{4}, 0$ & en & 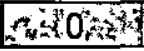 & 1 & $\ln x 0^{\circ}, 1$ & 4 \\
\hline$\times 3$ & 0 & 2 & 1 & -1 & 0 & 2 \\
\hline$x 1$ & 1 & -1 & 0 & 1 & 0 & 4 \\
\hline$\times 5$ & 0 & -1 & 0 & 1 & 1 & 8 \\
\hline
\end{tabular}

$\mathrm{D}=\{1,2\}$. Tome $\mathrm{k}=1$, assim $\mathrm{S}^{1}=\{2\}$. Observe que (P) é redutível com relação a $\mathrm{S}^{\mathbf{I}}$.

$\underline{i t}=1$

Tableau 1 - correspondente ao problema $\left(\mathrm{P}^{1}\right)$

\begin{tabular}{|c|c|c|c|c|c|c|}
\hline & $X_{1}$ & $x 2$ & $x 3$ & $\times 4$ & $\times 5$ & \\
\hline$z$ & 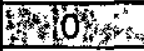 & ${ }_{4} O_{5}+k$ & 1,5 & 4 & 400 & 7 \\
\hline$x 2$ & 0 & 1 & 0,5 & $-0,5$ & 0 & 1 \\
\hline$x 1$ & 1 & 0 & 0,5 & 0,5 & 0 & 5 \\
\hline$\times 5$ & 0 & 0 & 0 & 1 & 1 & 8 \\
\hline
\end{tabular}

$D=\{2\}$, logo $k=2$ e como $S^{1}=\left(S^{0} \cup D\right\} \backslash\{k\}$, e o conjunto $S^{0}$ é vazio, segue que $\quad S^{1}=\varnothing$.

$i t=2$

Tableau 2 - correspondente ao problema $\left(\mathrm{P}^{2}\right)$

$D=\{4\}, k=4$ e $S^{1}=\varnothing$ 
$i t=3$

Tableau 3 - correspondente ao problema $\left(\mathrm{P}^{3}\right)$

\begin{tabular}{|c|c|c|c|c|c|c|}
\hline & $\times 1$ & $x 2$ & $\times 3$ & $\times 4$ & $x 5$ & \\
\hline $\bar{Z}$ & 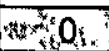 & 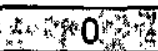 & 1,5 & 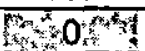 & 0,5 & 11 \\
\hline$x 2$ & 0 & 1 & 0,5 & 0 & 0,5 & 5 \\
\hline$x 1$ & 1 & 0 & 0,5 & 0 & $-0,5$ & 1 \\
\hline$\times 4$ & 0 & 0 & 0 & 1 & 1 & 8 \\
\hline
\end{tabular}

Como $\mathrm{D}=\varnothing$, a solução atual é ótima para o problema (P).

Uma outra regra, também recursiva, chama de regra criss-cross é baseada nos seguintes aspectos: enquanto busca a solução ótima, não necessariamente mantém a factibilidade primal e nem dual, pois pode-se encontrar um "caminho mais curto" para se chegar ao ótimo, quando se permite infactibilidade primal e dual.

O método criss-cross finito ou método auto-dual (Terlaky 1987) foi desenvolvido baseado na técnica récursiva de Bland (1977).

Um exemplo que exige um número exponencial de iterações quando esta regra é utilizada é dado por Roos (1990). A construção desse exemplo está relacionada com o exemplo que Avis e Chavatal (1978) deram para a regra do menor índice de Bland.

\subsection{O Algoritmo de Anstreicher e Terlaky}

Anstreicher e Terlaky (1994) publicaram o algoritmo do tipo simplex ("Monotonic Build-Up") (MBU), que tem propriedades teóricas interessantes. Os autores apresentam a versão primal e dual do algoritmo MBU.

O algoritmo primal inicia com uma solução básica factível e, qualquer variável não básica tendo custo reduzido negativo, a regra produz uma sequuência de iterações, tal que no final a variável não básica escolhida originalmente entra na base, e todos custos reduzidos que eram originalmente não negativos permanecem não negativos. A regra então constrói monotonicamente (estritamente crescente) uma base dual factível, e assim uma base ótima.

Existem duas propriedades interessantes na regra MBU. Primeiro, há dois testes da razão a cada iteração - ambas as variáveis, de entrada e de saída, são determinadas por estes testes. A dual factibilidade (para as variáveis que já são dual factíveis) é preservada durante o algoritmo, devido ao segundo teste da razão, enquanto a primal factibilidade é temporariamente perdida, mas é recuperada de tempo em tempo. 
Como a regra produz uma seqüência de iterações que resulta em bases intermediárias, que não são primal e nem dual factível, ela pode ser vista como um método de pontos exteriores.

Os autores consideraram o par primal-dual na forma padrão:

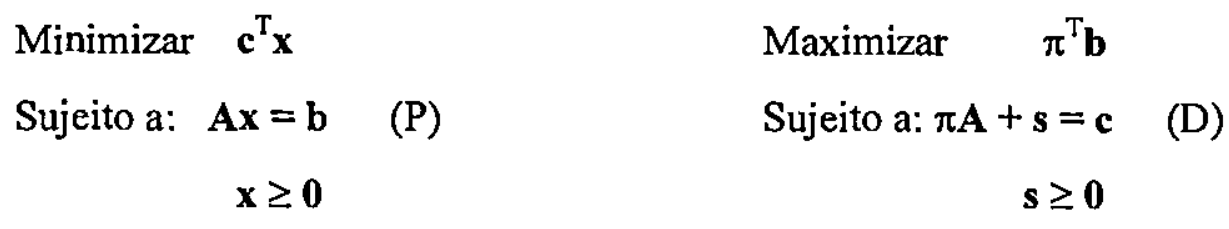

onde $\mathbf{A}$ é uma matriz $m \times n$, posto $(\mathbf{A})=m$.

Definição 3.1: uma variável será chamada de "variável de condução" se representa um papel especial na seleção do elemento pivô, isto é, a variável de saída (ou entrada) é posteriormente determinada usando esta linha (coluna).

Daremos agora, o algoritmo primal para a regra MBU. Em seguida apresentaremos a versão dual desta regra. Como no método primal simplex, suponha que esteja disponível uma solução básica primal factível inicial. A regra procede como se segue:

\section{MBU - primal}

$\operatorname{Passo0}-\operatorname{Seja} J^{+}=\left\{j: c_{\mathbf{j}}-\pi \mathbf{a}_{\mathrm{j}} \geq 0\right\} \quad J^{-}=\left\{j: c_{\mathbf{j}}-\pi \mathbf{a}_{\mathbf{j}}<0\right\}$

Passo 1 - Seja $k \in J^{-}$um índice arbitrário. (A variável $\mathrm{x}_{\mathrm{k}}$ será referida como a "variável de condução")

Se $J^{-}=\phi$, então a base atual é ótima.

Passo 2 - (Seleção da variável de saída)

Seja $r$ tal que: $\frac{\mathrm{x}_{\mathrm{Br}_{\mathrm{r}}}}{\mathrm{y}_{\mathrm{rk}}}=\min \left\{\frac{\mathrm{x}_{\mathrm{B}_{\mathrm{i}}}}{\mathrm{y}_{\mathrm{ik}}}: \mathrm{y}_{\mathrm{ik}}<0, i=1, \cdots, m\right\}$. (A variável $\mathrm{x}_{\mathrm{B}_{\mathrm{r}}}$ é a variável de saída)

Se não existe $r$, ou seja, se $\mathrm{y}_{\mathrm{ik}} \geq 0, i=1, \ldots, m$ então o problema é ilimitado, pare.

Passo 3 - (Seleção da variável de entrada)

$$
\text { Seja } s \text { tal que: } \frac{\mathrm{c}_{\mathrm{s}}-\pi \mathbf{a}_{\mathrm{s}}}{\mathrm{y}_{\mathrm{rs}}}=\min \left\{\frac{\mathrm{c}_{\mathrm{j}}-\pi \mathbf{a}_{\mathrm{j}}}{\mathrm{y}_{\mathrm{rj}}}: \mathrm{y}_{\mathrm{rj}}>0, j \in J^{+}\right\} \text {. (A variável } \mathrm{x}_{\mathrm{s}} \text { é a }
$$

variável de entrada) 
Se não existe $s$, então $s=k$

$\operatorname{Sejam} \theta_{1}=\frac{c_{3}-\pi a_{s}}{y_{r s}}$ e $\theta_{2}=\frac{c_{k}-\pi a_{k}}{y_{r k}}$

Passo 3a $-\operatorname{Se} \theta_{1}<\theta_{2}$, então faça pivoteamento em $(r, s), \mathrm{x}_{s}$ entra na base. Atualize $J^{+}=\left\{j: c_{j}-\pi \mathbf{a}_{\mathbf{j}} \geq 0\right\} \quad$ A “variável de condução" não é mudada. Vá ao passo 2.

Passo $3 \mathrm{~b}-\operatorname{Se} \theta_{1} \geq \theta_{2}$, então faça pivoteamento em $(r, k), \mathbf{x}_{\mathbf{k}}$ entra na base. Atualize $J^{+}=\left\{j: c_{\mathbf{j}}-\pi \mathbf{a}_{j} \geq 0\right\} \quad$ Vá ao passo 1 .

Note que no passo $3 \mathrm{~b}$, o conjunto de índices duais factíveis $J^{+}$é estritamente crescente. Por isso o algoritmo tem o nome monotonicamente construtivo. Observe também que durante a sequência de pivoteamento, enquanto a variável de condução está fixa, a base pode se tornar infactível. Interessantemente, o teste da razão no passo 2 é automaticamente restrito à parte factível da base. No passo $3 b$, quando a variável de condução entra na base, a primal factibilidade de toda ą base é restabelecida. Então sempre tem uma base factível no passo 1 . A prova destas afirmações é dada por Anstreicher (1990) e Anstreicher e Terlaky (1994).

Vamos apresentar a seguir a versão dual do MBU. Suponha que uma base dual esteja disponivel.

\section{MBU - Dual}

Passo $0-$ Seja $J^{+}=\left\{i: \mathrm{x}_{\mathrm{B}_{\mathrm{i}}} \geq 0\right\}, J^{-}=\left\{i: \mathrm{x}_{\mathrm{B}_{\mathrm{i}}}<0\right\}$

Passo 1 - Seja $k \in J^{-}$um índice arbitrário. (A variável $\mathrm{x}_{\mathrm{k}}$ será referida como a "variável de condução" linha)

Se $J^{-}=\phi$, então a base atual é ótima.

Passo 2 - (Seleção da variável de entrada)

Seja $s$ tal que: $\frac{c_{s}-\pi \mathbf{a}_{s}}{y_{k s}}=\min \left\{\frac{c_{j}-\pi \mathbf{a}_{j}}{y_{k j}}: y_{k j}>0\right\}$. (A variável $x_{s}$ é a variável de entrada)

Se não existe nenhum $s$, ou seja, se $\mathrm{y}_{\mathrm{kj}} \leq 0, j=1, \ldots, n$ então o problema dual é ilimitado e portanto primal infactivel. 
Passo 3 - (Seleção da variável de saída)

Seja $r$ tal que: $\frac{\mathrm{x}_{\mathrm{Br}}}{\mathrm{y}_{\mathrm{rs}}}=\min \left\{\frac{\mathrm{x}_{\mathrm{Bi}}}{\mathrm{y}_{\text {is }}}: \mathrm{y}_{\mathrm{is}}<0, i \in J^{+}\right\}$. (A variável $\mathrm{x}_{\mathrm{r}}$ é a variável de saída)

Se não existe $r$, então $r=k$

Seja $\theta_{1}=\frac{x_{B_{r}}}{y_{r s}}$ e $\theta_{2}=\frac{x_{B k}}{y_{k s}}$.

Passo $3 a-\operatorname{Se} \theta_{1}<\theta_{2}$, então faça pivoteamento em $(r, s), \mathrm{x}_{\mathrm{r}}$ sai da base. Atualize $J^{+}=\left\{i: \mathrm{x}_{\mathrm{B}_{\mathrm{i}}} \geq 0\right\} \quad \mathrm{A}$ "variável de condução" não é mudada. Vá ao passo 2 .

Passo $3 b-\operatorname{Se} \theta_{1} \geq \theta_{2}$, então faça pivoteamento em $(k, s), \mathrm{x}_{\mathrm{k}}$ sai da base. Atualize $J^{+}=\left\{i: \mathrm{x}_{\mathrm{Bi}} \geq 0\right\}$. Vá ao passo 1 .

\subsection{Outros Variantes Simplex}

Nesta seção descreveremos três métodos disponíveis da literatura, com características interessantes, que segundo os autores apresentam bons resultados computacionais quando comparados com o método clássico de Dantzig.

\subsubsection{Método PAPA (Pivot and Probe Algorithm)}

Sethi e Thompson (1984) propõem em seu artigo uma modificação no método simplex usual. Os autores trabalharam com problema linear (original) no seguinte formato:

$$
\begin{aligned}
& \text { Maximizar } \mathbf{c}^{\top} \mathbf{x} \\
& \text { Sujeito a: } \mathbf{A x}=\mathbf{b} \\
& x \geq 0
\end{aligned}
$$

onde $\mathbf{A}$ é uma matriz $m \times n$ e posto $(\mathbf{A})=m$. Eles supuseram: I) $\mathbf{b} \geq \mathbf{0}$ para o qual torna $\quad \mathbf{x}=$ 0 uma solução factível; 2) uma restrição de regularização da forma: $\sum_{j=1}^{n} \mathbf{x}_{\mathrm{j}} \leq M$ inclusa no conjunto de restrições, onde $M$ é um número muito grande. Tal restrição assegura que o problema acima é limitado. 
Os autores utilizaram a definição de restrição candidata, como sendo uma restrição tal que, pelo menos em uma iteração do algoritmo, contém um elemento pivô em potencial, ou seja, tal restrição será usada para identificar a linha pivô. Claramente restrições nãocandidatas não podem estar ativas na solução ótima do problema.

Num artigo anterior (Sethi e Thompson, 1983) foi observado que em problemas gerados aleatoriamente, somente 15 a $25 \%$ de todas as restrições são restrições candidatas e aproximadamente $70-90 \%$ delas estão ativas no ótimo.

Sethi e Thompson (1984) então, apresentaram uma forma de tirar proveito do fato que a maioria das restrições nunca são candidatas. O método começa resolvendo um problema linear relaxado consistindo das restrições do problema original que são inicialmente candidatas a serem ativas. Para isso utiliza-se do método simplex primal. A solução do problema relaxado é um limitante superior para o ótimo do problema original. Um segmento de linha que une a solução infactível e a factível é usado para identificar a restrição "mais violada"; ao mesmo tempo isto dá um limitante inferior para o problema original. Esta restrição é então adicionada ao problema relaxado, que é resolvido novamente, agora com o método dual simplex, o qual fornece um novo limitante superior, e este procedimento é feito até que não haja nenhuma restrição violada, obtendo assim, a solução ótima para o problema original.

A figura 3 ilustra um exemplo de como o método trabalha. Inicalmente as restrições 1 e 2 são candidatas. A solução ótima para o problema relaxado é o ponto A, obtido pelo método primal simplex. Depois de obter este vértice (solução) dual factível, o segmento une a origem (solução primal factível) e identifica a restrição 3 como sendo a mais violada, através do ponto B. Esta restrição depois de transformada é incluída no subproblema. O método dual simplex é utilizado para obter uma outra solução dual factível representada pelo ponto C. Um outro segemento ligando a origem e o ponto $\mathrm{C}$ informa que a restrição mais violada agora é a 7, através do ponto D. O método procede até que não haja restrições violadas. Assim, a solução ótima é o ponto E. 


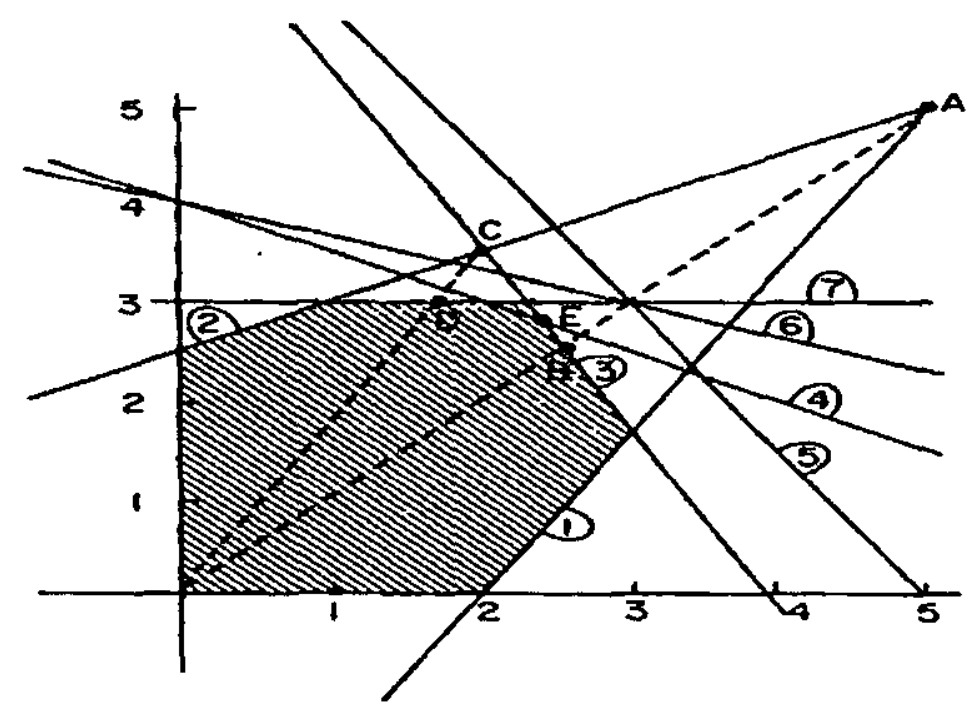

Figura 3 - O método PAPA graficamente

Segundo Sethi e Thompson há algumas razões por que este tipo de abordagem é computacionalmente benéfico. Primeiro, os problemas relaxados envolvem aproximadamente 10-20\% de todas as restrições, de forma que estes são problemas de programação linear relativamente simples, que podem ser resolvido mais rápido. Segundo, os segmentos de linha fornecem uma seqüência de soluções primal factíveis e limitantes superiores e inferiores, os quais podem ser usados para avaliar quão perto a solução corrente (primal factível) está da ótima. Terceiro, eles encontraram que na solução ótima, 80-90\% das restrições que estão na base, são ativas. Além disso, aproximadamente $90 \%$ das restrições do problema original que não estão ativas no ótimo, normalmente nunca foram utilizadas como linha pivô. Quarto, foi encontrado experimentalmente que somente 10-30 probes foram utilizadas para resolver problemas com dimensões superiores a $300 \times 310$, e que o esforço de tempo computacional é aproximadamente $20-40 \%$ do esforço exigido pelo método simplex. E ainda, as experiências computacionais dos autores, em problemas gerados aleatoriamente, indicam que 0 desempenho do novo método, geralmente melhora com o aumento nas dimensões do problema. Ressalta-se, porém, que o número de iterações realizadas pelo método PAPA com relação ao simplex (primal) é aproximadamente 1,5 vezes mais, já que o método PAPA utiliza os algoritmos primal e dual simplex como sub-rotinas.

\subsubsection{Nova Regra Primal e Dual de Pivoteamento}

Chen et al. (1994) apresentaram um algoritmo do tipo simplex para programação linear, que trabalha com soluções primal factível e dual factível associadas com bases que 
diferem somente por uma coluna. Segundo os autores, tal algoritmo é muito pouco afetado pela degeneração.

Os autores consideraram um programa linear na seguinte forma: $\max \left\{\mathbf{c}^{\mathrm{T}} \mathbf{x}: \mathbf{A x}=\mathbf{b}, \mathbf{x} \geq 0 \quad\right.$ (P) $\}$. Para construir o algoritmo, fizeram as seguintes considerações: Seja $\mathbf{B}_{\mathrm{p}}$ e $\mathbf{B}_{\mathrm{d}}$ bases primal e dual para o problema acima, respectivamente, onde se tem um caso especial, no qual $\mathbf{B}_{\mathrm{p}}$ e $\mathbf{B}_{\mathrm{d}}$ diferem exatamente por uma coluna. Seja $d$ (pertencente à matriz A) uma coluna de $\mathbf{B}_{d}$ que distingue $\mathbf{B}_{p}$ e $\mathbf{B}_{d}$ e seja $\mathbf{x}_{p}$ e $\mathbf{x}_{d}$ soluções básicas primal e dual factíveis, associadas a estas bases. Assim, eles utilizaram a proposição abaixo, como ponto chave do algoritmo.

Proposição 3.2. Se $x_{p}$ e $x_{d}$ são soluções não degeneradas e não ótimas, então somente uma das seguintes propriedades acontece:

i) A coluna $d$ pode entrar na base $B_{p}$ na próxima iteração primal, ou

ii) A coluna $d$ pode deixar a base $\mathbf{B}_{\mathbf{d}}$ na próxima iteração dual.

Como nosso propósito aqui não é detalhar o algoritmo em questão e sim observar o comportamento computacional do mesmo, apresentamos os resultados computacionais preliminares realizados pelos autores.

O algoritmo foi implementado utilizando o software Matlab e comparado com método simplex com a regra do menor custo reduzido (regra de Dantzig), que foi também implementado no Matlab.

Foram gerados aleatoriamente vinte problemas testes na seguinte forma

$$
\max \left\{\tilde{\mathbf{c}}^{\mathrm{T}} \widetilde{\mathbf{x}}: \tilde{\mathbf{A}} \tilde{\mathbf{x}} \leq \tilde{\mathrm{b}}, \tilde{\mathbf{x}} \geq 0\right\}
$$

onde $\tilde{\mathbf{A}}$ tem dimensões $\tilde{m} \times \tilde{n}$. Todas as restrições $\tilde{\mathbf{A}} \widetilde{\mathbf{x}} \leq \tilde{\mathbf{b}}$ foram tangente a uma bola unitária, de centro na origem ou um ponto com duas unidades distante da mesma. Nenhuma das restrições foram redundantes.

$O$ vértice inicial foi à origem. Portanto a posição do centro determina se o vértice inicial é factível (primal) ou não. Como $\tilde{m}>\tilde{n}$ e o algoritmo foi implementado para resolver o problema $(\mathrm{P})$, os dados de $(\widetilde{\mathrm{P}})$ foram transformados para a forma padrão: $\left\{\max \mathbf{c}^{\mathrm{T}} \mathbf{x}, \mathbf{A x}=\mathbf{b}\right.$, $\mathbf{x} \geq 0\}$, onde A é $m \times n, \operatorname{com} m=\tilde{m}$, e $n=\tilde{m}+\tilde{n}$. Assim, para o menor e o maior dos problemas A foi $25 \times 75$ e $150 \times 350$, respectivamente.

A tabela 3.1 apresenta os resultados obtidos pelos autores. 
Tabela 3.1

\begin{tabular}{|c|c|c||c|c|c|c|}
\hline & \multicolumn{1}{|c||}{} & \multicolumn{1}{|c||}{} & \multicolumn{3}{c|}{ Número de iteraçס para duas regras } \\
\hline $\bar{m}$ & $\bar{n}$ & densidade & \multicolumn{2}{c|}{ Origem factivel } & \multicolumn{2}{c|}{ Origem infactivel } \\
\hline & & & MSU & MP & MSU & MP \\
\hline 50 & 25 & 0,3 & 38 & 37 & 36 & 26 \\
\hline 100 & 25 & 0,3 & 41 & 42 & 32 & 26 \\
\hline 150 & 25 & 0,3 & 29 & 24 & 53 & 54 \\
\hline 200 & 25 & 0,3 & 51 & 41 & 38 & 38 \\
\hline 100 & 50 & 0,1 & 51 & 40 & 60 & 45 \\
\hline 150 & 50 & 0,1 & 102 & 81 & 114 & 92 \\
\hline 200 & 50 & 0,1 & 103 & 96 & 118 & 87 \\
\hline 150 & 100 & 0,1 & 260 & 179 & 292 & 207 \\
\hline 200 & 100 & 0,1 & 261 & 166 & 397 & 311 \\
\hline 200 & 150 & 0,1 & 454 & 308 & 430 & 264 \\
\hline
\end{tabular}

onde MSU $\rightarrow$ método simplex com a regra usual (Dantzig) e MP $\rightarrow$ método proposto pelos autores.

Observa-se que este novo método teve um desempenho relativamente um pouco melhor do que o método simplex usual. No maior dos problemas o método simplex efetuou $2,27 m$ iterações enquanto o método em questão chegou ao ótimo após $1,54 m$ iterações. $O$ mesmo comportamento pode ser observado para a fase II. Ou seja, estes testes preliminares indicam que o novo método tem um aumento no desempenho à medida que o problema cresce, comparado com o método simplex com a regra de Dantzig.

\subsubsection{Regra de Dantzig Normalizada}

Esta regra é conhecida desde os experimentos de Kuhn e Quandt (1963) e Wolfe e Cuttler (1963) e consiste em normalizar as direções simplex no cálculo dos custos relativos. Em geral, o algoritmo simplex com esta regra realiza menos iterações do que outros variantes do método simplex primal. Porém, por causa de sua implementação direta, foi inicialmente rejeitada como impraticável.

No entanto, Goldafarb e Reid (1977) publicaram o primeiro algoritmo do tipo simplex com a regra de Dantzig normalizada praticável. Os autores reportaram testes computacionais pré liminaré, mostrando a superioridade do algoritmo simplex com esta regra em relação ao algoritmo simplex com a regra de Dantzig.

Em vez de selecionar a variável com maior custo reduzido (regra de Dantzig), escolhese $o$ indice da variável que tem maior custo reduzido sobre $\gamma_{\mathrm{j}}$, isto é, $\frac{\mathbf{c}_{\mathrm{j}}-\pi \mathbf{a}_{\mathrm{j}}}{\gamma_{\mathrm{j}}}$, onde $\gamma_{\mathrm{j}}=\left\|\mathrm{d}^{\mathrm{j}}\right\|=\sqrt{1+\sum_{\mathrm{r}=1}^{\mathrm{m}} \mathrm{y}_{\mathrm{rj}}^{2}}$ e $\mathrm{d}^{\mathrm{j}}=\left[\frac{-\mathbf{B}^{-1} \mathbf{a}_{j}}{\mathbf{e}_{\mathrm{j}}}\right]$, sendo $\mathbf{e}_{j}$ a $j$-ésima 
coluna da matriz identidade de dimensão $(n-m)$ e $\mathbf{a}_{j}$ é a j-ésima coluna de $\mathbf{A}$, que faz parte da partição não básica, ou seja, $\mathrm{a}_{\mathrm{j}} \in \mathrm{N}$. Ou ainda $\mathrm{d}^{j}=\eta_{j}=\left[\frac{-\mathrm{B}^{-1} \mathbf{N}}{\mathrm{I}}\right] \mathbf{e}_{j-m}, \quad j=m+1, \ldots, n$. Portanto, veja que $\gamma_{\mathrm{j}}$ é a norma euclidiana do vetor direção ao longo da aresta associada com o aumento na variável não básica $x_{j}$ por unidade. Assim $\frac{\mathbf{c}^{\mathbf{T}} \mathbf{d}^{\mathrm{j}}}{\left\|\mathbf{d}^{\mathrm{j}}\right\|}=\frac{\mathrm{c}_{\mathrm{j}}-\pi \mathbf{a}_{\mathrm{j}}}{\gamma_{\mathrm{j}}}$ é a taxa de variação no valor da função objetivo por unidade de distância alongo da direção simplex. A fim de evitar esforço computacional por iteração, a quantidade $\gamma_{j}$ precisar ser atualizada em lugar de ser recalculada de uma iteração para próxima. Daremos a seguir as fórmulas de atualizações.

Para problemas de grande porte, o cálculo explícito de todas as normas $\left\|\gamma_{\mathrm{j}}\right\|, \quad j \in \mathrm{N}$, em cada iteração é proibitivamente caro. Assim, para implementar esta regra de pivoteamento de uma maneira prática, Goldfarb e Reid (1977) derivaram fórmulas de recorrência para atualizar os valores de $\gamma_{\mathrm{j}}$, para todos os índices não básicos $\mathrm{j}$, depois de cada pivoteamento. Se a variável $x_{k}$ substitui a variável $x_{t}$ na base, então tem-se as seguintes fórmulas de recorrência:

$$
\begin{gathered}
\bar{\eta}_{r}=-\frac{\eta_{k}}{\alpha_{k}}, \\
\bar{\eta}_{j}=\eta_{i}-\eta_{k} \bar{\alpha}_{j}, \quad j \in \mathrm{N}, j \neq k
\end{gathered}
$$

Assim temos que:

$$
\begin{gathered}
\bar{\gamma}_{r}=\frac{\gamma_{k}}{\alpha_{k}^{2}} \\
\bar{\gamma}_{j}=\gamma_{j}-2 \bar{\alpha}_{j} \alpha_{j}^{T} v+\bar{\alpha}_{j}^{2} \gamma_{k}, j \in \mathrm{N}, j \neq k,
\end{gathered}
$$

onde $\alpha_{j}=\sigma^{\mathrm{T}} \mathbf{a}_{\mathrm{j}}, \overline{\alpha_{\mathrm{j}}}=\frac{\alpha_{\mathrm{j}}}{\alpha_{\mathrm{k}}}, \mathrm{Bw}=\mathbf{a}_{\mathrm{k}}, \mathrm{B}^{\mathrm{T}} \mathbf{v}=\mathbf{w}$, e $\mathbf{B}^{\mathrm{T}}=\mathbf{e}_{\mathrm{r}}$. Observe que $\alpha_{k}=w_{r}$, e que $\alpha e \bar{\alpha}$ são as componentes de $r$-ésima linha de $\mathbf{B}^{-1} \mathbf{A} e \overline{\mathbf{B}}^{-1} \mathbf{A}$, respectivamente, onde $\overline{\mathbf{B}}$ é nova matriz base após o pivoteamento.

Multiplicando as duas primeiras equaçoes acima por $\mathbf{c}^{\mathbf{T}}$, tem-se que os números $\bar{\alpha}_{j}$ são também necessários se os custos reduzidos são atualizados. Também $\bar{\gamma}_{r}$ pode ser calculado explicitamente, já que $\gamma_{k}=1+\mathbf{w}^{T} \mathbf{w}$. 
A atualização de requer alguns cálculos extra: a solução de uma equação a mais e o produto interno de $\mathbf{v}$ com as colunas $\mathbf{a}_{\mathbf{j}}$ não básicas correspondendo aos que são diferentes de zero.

Goldfarb e Reid (1992) com suas experiências computacionais, apresentadas a seguir, observaram que o aumento no tempo computacional por iteração com relação ao método simplex usual é aproximadamente $8 \%$, devido a esses cálculos adicionais.

Os autores compararam o algoritmo simplex com a regra de Dantzig normalizada, utilizando-se fatoração LU, com o algoritmo simplex usando a regra usual (regra de Dantzig). Os algoritmos foram implementados com opções no SSLV, que é uma coleção de rotinas simplex (FORTRAN e C), que faz parte do Optimization System Library (OSL) produzido pela IBM (1991). Todos os resultados relatados foram obtidos em uma Workstation da IBM. Essas rotinas forneceram uma implementação robusta e estável do método simplex.

Antes de resolver o problema original, foi feita uma redução do mesmo por um "préprocessador" para remover linhas redundantes e eliminar ou fixar variáveis quando possível.

$\mathrm{Na}$ média os algoritmos implementados com a regra de Dantzig normalizada foi superior ao algoritmo simplex usual (que utilizou custo parcial; ver capitulo VI) com relação ao número de iterações.

Os problemas foram obtidos de um conjunto de problemas teste de programação linear (Lustig, 1989). A forma de cada problema foi:

$$
\begin{aligned}
& \text { Minimizar } \mathbf{c}^{\mathrm{T}} \mathbf{x} \\
& \text { Sujeito a: } \mathbf{A x} \diamond \mathbf{b} \\
& \mathbf{r} \leq \mathbf{x} \leq \mathrm{s}
\end{aligned}
$$

onde $A \in R^{m \times n}$, e o símbolo $\diamond$ indica que cada linha é relacionada ao elemento $b_{i}$ correspondente por uma relação de menor ou igual $(\leq)$, maior ou igual $(\geq)$ ou de igualdade $(=)$.

Os autores observaram que alguns problemas tiveram mais de 10000 elementos diferentes de zero na matriz de restrições antes de aplicar o pré-processador. Problemas de grande porte e dificeis foram resolvidos como, por exemplo: um problema de programação de tripulação, um problema de designação e dois de programação da frota de navios.

Os problemas em média tiveram 3412 linhas e 12492 colunas, sendo que o maior deles foi de 10662 × 76090, e considerando o fato que o pré-processador já tinha sido aplicado. $O$ algoritmo com a regra de Dantzig normalizada foi significativamente muito superior ao 
algoritmo simplex usual tanto para o método primal, quanto para o dual. Para os 20 problemas utilizados pelos autores, o tempo (em horas) para resolvê-los usando o algoritmo de Dantzig primal e dual foi respectivamente 110,19 e 177,78 enquanto o algoritmo primal e dual proposto foi 3,89 e 6,36, o que é muito impressionante a diferença. Excluindo 5 problemas onde o número de iterações foi expressivamente alto, a média para o algoritmo de Dantzig foi 6,3m enquanto o algoritmo com a regra de Dantzig normalizada teve uma média $1,9 m$ iterações. O mesmo comportamento foi observado quando se tratou do tempo computacional.

Portanto, o variante simplex com a regra de Dantzig normalizada foi claramente superior ao método simplex usual, para resolver problemas difíceis de programação linear de grande porte. Isto indica que há, ainda, campo de pesquisa em algoritmos tipo simplex. 


\section{Capítulo IV}

\section{Programa Linear com Variáveis Canalizadas}

\subsection{O Problema Dual Linear por Partes}

Nesta seção deduzimos o problema dual a partir do problema primal com restrições canalizadas. Tais restrições surgem em muitos problemas práticos, uma vez que tolerâncias são usuais tanto em demandas, ou normas técnicas e etc. O problema dual é linear por partes e o algoritmo dual simplex é então especializado.

Considere o seguinte problema primal canalizado na forma padrão:

$$
\begin{aligned}
& \text { Minimizar } Z(x)=c^{\top} x \\
& \text { Sujeito a : } \mathbf{A x}=\mathbf{b} \\
& \mathbf{r} \leq \mathrm{x} \leq \mathrm{s}
\end{aligned}
$$

onde $A \in \mathrm{R}^{m \times n} ; \mathbf{r}, \mathbf{s} \in \mathrm{R}^{n}$ com $\mathrm{r}_{\mathrm{i}} \leq \mathrm{s}_{\mathrm{i}} ; \mathbf{c}, \mathbf{x} \in \mathrm{R}^{n}$. Suporemos sem perda de generalidade que posto(A) $=m$. Podemos construir o problema dual como se segue:

Defina a função Lagrangiana da seguinte maneira:

Para cada $\lambda \in \mathrm{R}^{m}$ defina

$$
\begin{aligned}
L(\mathbf{x}, \lambda) & =\mathbf{c}^{\mathrm{T}} \mathbf{x}+\lambda^{\mathrm{T}}(\mathbf{b}-\mathbf{A} \mathbf{x}) \\
& =\left(\mathbf{c}^{\mathrm{T}}-\lambda^{\mathbf{T}} \mathbf{A}\right) \mathbf{x}+\lambda^{\mathrm{T}} \mathbf{b}
\end{aligned}
$$


Então a função dual é definida por:

$$
\begin{array}{ll}
h(\lambda)=\underset{r}{\mathbf{m i n}}\{\mathbf{x} \leq \mathbf{s} & \mathbf{r} \leq \mathbf{x} \leq \mathbf{s}
\end{array}
$$

O problema (4.3) pode ser rescrito como

$$
\begin{gathered}
\mathrm{h}(\lambda)=\min \left\{\left(\mathbf{c}^{\mathrm{T}}-\lambda^{\mathrm{l}} \mathbf{A}\right) \mathbf{x}\right\}+\lambda^{\mathrm{T}} \mathbf{b}, \\
\mathbf{r} \leq \mathbf{x} \leq \mathbf{s}
\end{gathered}
$$

já que $\lambda^{\mathrm{T}} \mathbf{b}$ é uma constante para cada $\lambda \in \mathrm{R}^{m}$. Assim o problema Lagrangiano é:

$$
\begin{array}{cc}
h(\lambda) & =\min \left\{\sum_{\mathrm{i}=1}^{\mathrm{n}}\left(\mathrm{c}_{\mathrm{i}}-\lambda^{\mathrm{T}} \mathbf{a}_{\mathrm{i}}\right) \mathrm{x}_{\mathrm{i}}\right\}+\lambda^{\mathrm{T}} \mathbf{b}=\sum_{\mathrm{i}=1}^{\mathrm{n}} \min \left\{\left(\mathrm{c}_{\mathrm{i}}-\lambda^{\mathrm{T}} \mathbf{a}_{\mathrm{i}}\right) \mathrm{x}_{\mathrm{i}}\right\}+\lambda^{\mathrm{T}} \mathbf{b} \\
\mathrm{r}_{\mathrm{i}} \leq \mathrm{x}_{\mathrm{i}} \leq \mathrm{s}_{\mathrm{i}} \quad i=1, \ldots, n & \mathrm{r}_{\mathrm{i}} \leq \mathrm{x}_{\mathrm{i}} \leq \mathrm{s}_{\mathrm{i}} \quad i=1, \ldots, n
\end{array}
$$

uma vez que os subproblemas nas variáveis $x_{i}$ são independentes.

Assim, a minimização em (4.4) é obtida pela solução de cada subproblema:

$$
\begin{aligned}
& \min \left\{\left(c_{i}-\lambda^{T} a_{i}\right) x_{i}\right\}= \begin{cases}\left(c_{i}-\lambda^{T} a_{i}\right) s_{i} & \text { se }\left(c_{i}-\lambda^{T} a_{i}\right)<0 \\
0 & \text { se }\left(c_{i}-\lambda^{T} a_{i}\right)=0 \\
\left(c_{i}-\lambda^{T} a_{i}\right) r_{i} & \text { se }\left(c_{i}-\lambda^{T} a_{i}\right)>0\end{cases} \\
& \quad r_{i} \leq x_{i} \leq s_{i}
\end{aligned}
$$

Defina: $\mu^{\mathrm{T}}=\mathbf{c}^{\mathrm{T}}-\lambda^{\mathrm{T}}$ A e a função $\theta_{\mathrm{i}}\left(\mu_{\mathrm{i}}\right)$ como sendo:

$$
0_{i}\left(\mu_{i}\right)=\left\{\begin{array}{ll}
\mu_{i} s_{i}, & \text { se } \mu_{i}<0 \\
0, & \text { se } \mu_{i}=0 \\
\mu_{i} r_{i}, & \text { se } \mu_{i}>0
\end{array} \quad\right. \text { (função linear por partes) }
$$

Portanto o problema dual é definido por:

$$
\operatorname{Maximizar} h(\lambda, \mu)=\sum_{i=1}^{n} \theta_{i}\left(\mu_{i}\right)+\lambda^{T} \mathbf{b}
$$

Sujeito a: $\quad \mathbf{A}^{\mathrm{T}} \lambda+\mu=\mathbf{c}$

$\mu$ irrestrito de sinal. 


\subsection{Método Dual Simplex Para Variáveis Canalizadas}

Descreveremos aqui o método dual simplex que tem uma inicialização imediata (uma base qualquer) e percorre vértices infactíveis da região de factibilidade do problema primal, satisfazendo as condições de otimalidade.

Considere uma partição sobre as linhas de $\mathbf{A}^{\mathrm{T}}: \mathbf{A}^{\mathrm{T}}=\left(\frac{\mathbf{B}^{\mathrm{T}}}{\mathbf{N}^{\mathrm{T}}}\right)$ com $\mathbf{B} \in \mathrm{R}^{m \times m}$ e posto $(B)=m$. Esta partição impõe uma partição nas restrições dual em (4.6), portanto:

$$
\left(\frac{\mathbf{B}^{\mathrm{T}}}{\mathbf{N}^{\mathrm{T}}}\right) \lambda+\left(\frac{\underline{\mu}_{\mathrm{B}}}{\mu_{\mathrm{N}}}\right)=\left(\frac{\mathbf{c}_{\mathrm{B}}}{\mathbf{c}_{\mathrm{N}}}\right)
$$

ou

$$
\begin{aligned}
& \mathbf{B}^{\mathrm{T}} \lambda+\mu_{\mathrm{B}}=\mathbf{c}_{\mathrm{B}} \quad \text { (restrições básicas) } \\
& \mathbf{N}^{\mathrm{T}} \lambda+\mu_{\mathrm{N}}=\mathbf{c}_{\mathrm{N}} \quad \text { (restrições não básicas) }
\end{aligned}
$$

Para qualquer escolha de $\mu_{\mathrm{B}}$, o sistema fica unicamente determinado. Em particular, $\hat{\mu}_{B}=0, \hat{\lambda}^{T}=c_{B}^{T} B^{-1}$ (multiplicador simplex) e $\hat{\mu}_{N}^{T}=c_{N}^{T}-\hat{\lambda}^{T} N$ (custos relativos) é chamada solução básica dual. Como $\mu$ é irrestrito de sinal, esta solução é factivel

A estratégica dual simplex consiste na perturbação de uma solução dual, de modo que apenas uma variável de folga dual associada a uma restrição básica seja alterada. Assim temos 2 casos, onde a figura 4.1 ilustra a situação:

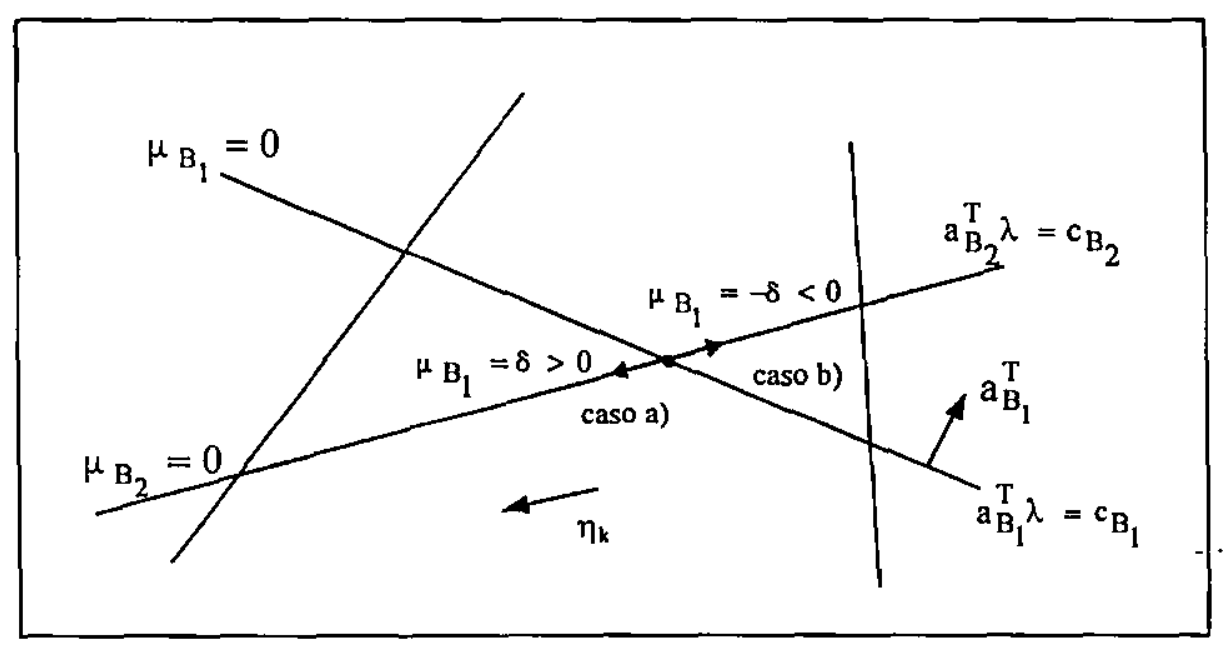

Figura 4.1 - Perturbação dual simplex 
Caso a) $\mu_{\mathrm{B}}=\hat{\mu}_{\mathrm{B}}+\delta \mathrm{e}_{\mathrm{k}} \Rightarrow \mu_{\mathrm{B} \mathrm{k}}=\delta \geq 0$, onde $B_{k}$ denota o índice da $k$-ésima variável básica.

Como $\mu_{\mathrm{Bk}}=\delta \geq 0$ de (4.5) temos que a contribuição para a função objetivo será:

$$
\theta_{B_{k}}\left(\mu_{B_{k}}\right)=r_{B_{k}} \delta \text {. }
$$

De (4.7) o valor da solução básica será:

$$
\lambda=\hat{\lambda}+\delta \eta_{k}
$$

onde $\eta_{k}$ é a $k$-ésima linha de $\left(-\mathbf{B}^{-1}\right)^{\mathrm{T}}$ que chamamos de direção dual simplex.

Considere agora, a seguinte partição no conjunto das variáveis não básicas $\mathrm{N}=\mathrm{N}^{+} \cup \mathrm{N}^{-}$, com $\mathrm{N}^{+}=\left\{j \in \mathrm{N} \mid \hat{\mu}_{\mathrm{N}_{\mathrm{j}}}>0\right\}$ e $\mathrm{N}^{-}=\left\{j \in \mathrm{N} \mid \hat{\mu}_{\mathrm{N}_{\mathrm{j}}}<0\right\}$. Por simplicidade supomos a solução dual não degenerada (o caso degenerado $\hat{\mu}_{N_{j}}=0$, pode ser acomodado em qualquer destes conjuntos). O valor da função do dual para a solução básica é dado por:

$$
h(\hat{\lambda}, \hat{\mu})=\sum_{j \in N^{+}} r_{N_{j}} \hat{\mu}_{N_{j}}+\sum_{j \in N^{-}} s_{N_{j}} \hat{\mu}_{N_{j}}+\hat{\lambda}^{T} \mathbf{b} .
$$

Logo para $\delta \geq 0$ e pequeno temos que o valor da função objetivo dual para a nova solução $\lambda$ será:

$$
h(\lambda, \mu)=h(\hat{\lambda}, \hat{\mu})+\delta\left(r_{B_{k}}-\hat{x}_{B_{k}}\right)
$$

Se $\hat{x}_{B_{k}}<r_{B_{k}}$, ou seja, se o limitante inferior for violado tem-se que a direção dual simplex é de subida. Então pode-se determinar o valor de $\delta$ que provoca mudança de sinal em $\mu_{\mathrm{N}}$ da seguinte maneira:

De (4.8) tem-se que

$$
\mu_{N_{i}}=\hat{\mu}_{N_{i}}-\delta\left(a_{N_{i}}^{T} \eta_{k}\right)
$$

Então se $\hat{\mu}_{N_{i}}$ e $a_{N_{i}}^{T} \eta_{k}$ têm o mesmo sinal, então $\mu_{N_{i}}$ pode trocar de sinal com o crescimento de $\delta$, logo

$$
\delta \leq \delta_{i}=\frac{\mu_{N_{i}}}{a_{N_{i}}^{T} n_{k}} .
$$

Seja $p$ um índice não básico tal que: 


$$
\delta_{p}=\frac{\hat{\mu}_{N_{p}}}{\mathbf{a}_{N_{p}}^{T} \eta_{k}}=\min \left\{\frac{\hat{\mu}_{N_{i}}}{\mathbf{a}_{N_{i}}^{T} \eta_{k}} \text { tal que } \frac{\hat{\mu}_{N_{i}}}{\mathbf{a}_{N_{i}}^{T} \eta_{k}} \geq 0 i=1, \ldots, m\right\} .
$$

Para $0 \leq \delta \leq \delta_{p}$ temos que: $h(\lambda, \mu)=h(\hat{\lambda}, \hat{\mu})+\delta\left(r_{B_{k}}-\hat{x}_{B_{k}}\right)$.

Quando $\delta=\delta_{p}$, de (4.9) e (4.10) $\Rightarrow \mu_{N_{p}}=0$. Isto é, a $p$-ésima restrição não básica torna-se ativa, enquanto $\mu_{\mathrm{B}_{\mathrm{k}}}=\delta$ faz com que a $k$-ésima restrição básica fique inativa.

Isto sugere uma nova base com índice $B_{k} \leftrightarrow N_{p}$.

Caso b) $\mu_{B}=\hat{\mu}_{B}-\delta e_{k} \Rightarrow \mu_{B k}=-\delta \leq 0$

Como $\mu_{\mathrm{Bk}}=-\delta \leq 0 \mathrm{de}(4.5)$ vem que:

$$
\theta_{B_{k}}\left(\mu_{B_{k}}\right)=s_{B_{k}} \delta
$$

Então o novo valor da solução básica será:

$$
\lambda=\hat{\lambda}-\delta \eta_{\mathrm{k}}, \quad\left(\eta_{\mathrm{k}}=-\left(\mathbf{B}^{-1}\right)^{\mathrm{T}} \mathrm{e}_{\mathrm{k}}\right)
$$

Logo para $\delta \geq 0$ e pequeno temos que o valor da função objetivo dual para nova solução $\lambda$ será:

$$
h(\lambda, \mu)=h(\hat{\lambda}, \hat{\mu})+\delta\left(\hat{x}_{B_{k}}-s_{B_{k}}\right) .
$$

Se $\hat{\mathrm{x}}_{\mathrm{B}_{\mathrm{k}}}>\mathrm{s}_{\mathrm{B}_{\mathrm{k}}}$, ou seja, se o limitante superior for violado tem-se que a direção dual simplex é de subida. De'(4.8) tem-se que

$$
\mu_{N_{i}}=\hat{\mu}_{N_{i}}+\delta\left(a_{N_{i}}^{T} \eta_{k}\right)
$$

Se $\hat{\mu}_{N_{i}}$ e $\mathbf{a}_{N_{i}}^{T} \eta_{k}$ têm sinais opostos, então $\mu_{N_{i}}$ troca de sinal quando $\delta$ cresce, portanto

$$
\delta \leq \delta_{i}=-\frac{\hat{\mu}_{N_{i}}}{\mathbf{a}_{N_{i}}^{T} \eta_{k}} .
$$

Seja $p$ um índice não básico tal que:

$$
\delta_{p}=-\frac{\hat{\mu}_{N_{p}}}{\mathbf{a}_{N_{p}}^{T} \eta_{k}}=\min \left\{-\frac{\hat{\mu}_{N_{i}}}{\mathbf{a}_{N_{i}}^{T} \eta_{k}} \text { tal que } \frac{\hat{\mu}_{N_{i}}}{\mathbf{a}_{N_{i}}^{T} \eta_{k}} \geq 0 i=1, \ldots, m\right\}
$$

Assim, para $0 \leq \delta \leq \delta_{\mathrm{p}}$ temos que: $\mathrm{h}(\hat{\lambda}, \mu)=h(\hat{\lambda}, \hat{\mu})+\delta\left(\hat{\mathrm{x}}_{\mathrm{B}_{\mathrm{k}}}-s_{\mathrm{B}_{\mathrm{k}}}\right)$ 
$\operatorname{com} \hat{\mathrm{x}}_{\mathrm{B}_{\mathrm{k}}}<\mathrm{s}_{\mathrm{B}_{\mathrm{k}}}$. Analogamente ao caso anterior, isto sugere uma nova base com $\mathrm{o}$ índice $B_{k} \leftrightarrow N_{p}$

Temos agora o seguinte teorema caracteriza uma solução ótima.

Teorema 4.1. Considere uma partição básica dual factível $\mathbf{A}=(\mathbf{B}, \mathbf{N})$ e as soluções básicas associadas:

Solução dual: $\quad \hat{\lambda}^{T}=\mathbf{c}_{B}^{T} B^{-1}, \hat{\mu}_{N}^{T}=\mathbf{c}_{N}^{T}-\hat{\lambda}^{\top} \mathbf{N}$,

Solução primal: $\quad \hat{\mathbf{x}}_{N_{j}}=\left\{\begin{array}{ll}s_{N_{j}} & \text { se } \hat{\mu}_{N_{j}}<0 \\ r_{N_{j}} & \text { se } \hat{\mu}_{N_{j}}>0\end{array}\right.$ e $\hat{\mathbf{x}}_{B}=\mathbf{B}^{-1} \mathbf{b}-\mathbf{B}^{-1} \mathbf{N} \hat{\mathbf{x}}_{N}$.

Se $\mathbf{r}_{\mathrm{B}} \leq \hat{\mathbf{x}}_{\mathrm{B}} \leq \mathrm{s}_{\mathrm{B}}$ então as soluções básicas primal e dual são ótimas para o problema primal e dual respectivamente.

Esse procedimento descreve o método dual simplex para o problema primal canalizado, onde o dual é linear por partes.

$\mathrm{O}$ método dual que apresentamos aqui tem uma vantagem sobre o método primal o qual omitimos sua apresentação, é quanto a inicialização, pois uma solução com característica de ótima é simples de ser obtida. No entanto, uma solução factível somente será alcançada na última iteração (solução ótima), ao passo que o método primal trabalha sempre com soluções factíveis, as quais são melhoradas a cada iteração. Se o problema for de grande porte, e a execução do algoritmo interrompida antes que uma solução ótima seja alcançada, então o método primal fornecerá uma solução factível melhorada (supondo que o tempo de busca de uma solução inicial tenha sido superado), enquanto que o método dual fornecerá uma solução infactível.

Esta estratégia dual simplex, baseada na característica linear por partes da função dual, pode ser facilmente estendida para o problema com restrições canalizadas:

$$
\begin{array}{ll}
\text { Minimizar } & Z(\mathbf{x})=\mathbf{c}^{\mathbf{T}} \mathbf{x} \\
\text { Sujeito a: } & \mathbf{e} \leq \mathbf{A x} \leq \mathbf{d} \\
& \mathbf{r} \leq \mathbf{x} \leq \mathbf{s}
\end{array}
$$

uma vez que este problema pode șer escrito por: 


\section{Minimizar $Z(x)=c^{\top} \mathbf{x}$}

Sujeito a: $\mathbf{A x}-\mathbf{y}=\mathbf{0}$

$$
\mathbf{r} \leq \mathbf{x} \leq \mathrm{s}, \mathrm{e} \leq \mathrm{y} \leq \mathrm{d} \text {. }
$$

Ressalta-se porém, que os testes computacionais realizados com este algoritmo especializado para restrições canalizadas, não foram para problemas de grande porte, onde o maior delés teve dimensão $400 \times 400$, ou seja, sempre conseguimos a solução ótima com um tempo de processamento razoável. No capítulo $\mathrm{V}$ encontram-se os resultados da implementação. 


\section{Capítulo V}

\section{Resultados Computacionais}

\subsection{Introdução}

Daremos a seguir os resultados computacionais realizados durante o desenvolvimento deste trabalho. Inicialmente tem-se os resultados para método simplex aplicado ao problema de corte em estoque, introduzido no capítulo I. Em seguida encontram-se os resultados para os métodos dual e primal simplex, onde são feitas algumas comparações do método dual simplex baseado em penalidad̉es lineares (Arenales, 1984) com o software Cplex 4.0 que contém pacote de sub rotinas de otimização. Antes de apresentarmos os resultados, considere a função $f(m)$ definida num exemplar $(m, n, \mathbf{c}, \mathbf{A}, \mathbf{b})$ de um problema de programação linear que denota o número de iterações para resolver o problema, em função de $m$, onde $m$ é o número de restrições deste exemplar.

\subsection{O Método Simplex Aplicado ao Problema de Corte}

Considere a forma matricial do problema de corte de estoque bidimensional inteiro, introduzido na seção 2.4 do capítulo I:

Minimizar $Z(\mathbf{x})=\mathbf{c}^{\mathrm{T}} \mathbf{x}$

Sujeito a : $\mathbf{A x} \geq \mathbf{d}$

$$
\mathbf{x} \geq \mathbf{0} \text { e inteiro }
$$


Para resolver o problema acima, o método simplex com geração de colunas foi utilizado relaxando-se a condição de integralidade.

Com isto o método encontra inicialmente uma solução ótima para esta relaxação. Este passo gera uma solução geralmente não-inteira $\overline{\mathbf{x}}$. Há vários procedimentos heurísticos que determinam uma solução inteira na 'vizinhança' desta solução (Pinto, 1999). Porém, como foi dito no capítulo II, não vamos tratar desse assunto aqui, pois nosso objetivo é observar o desempenho computacional do método simplex aplicado nesta classe de problemas.

A implementação foi feita por Pinto (1999) na linguagem pascal, utilizando turbo pascal 7.0. Os exemplos foram rodados em um microcomputador pentium $200 \mathrm{MHz}$, com 16 Mbytes de RAM.

Os dados foram gerados da seguinte maneira:

Dimensões das placas em estoque: $O$ tamanho das peças em estoque foram fixas em $L \times W=100 \times 100$.

Quantidade de itens a serem produzidos: Para realização da análise do método, foram utilizados os diferentes valores de $m: m=5,10,20$ e 40 . ( $m$ é o número de tipos de itens demandados)

Tamanho dos itens: Para cada exemplo rodado, os tamanhos $l_{i} \times w_{i}, i=1, \ldots, m$ foram gerados de forma aleatória. Conforme Morábito (1992), dividimos os problemas em classes, com relação ao tamanho dos itens, da seguinte maneira:

- 'exemplos pequenos' $\Rightarrow\left\{\begin{array}{l}l_{i} \in[0.25 \mathrm{~L}, 0.75 \mathrm{~L}] \\ w_{i} \in[0.25 \mathrm{~W}, 0.75 \mathrm{~W}]\end{array}, \quad i=1, \ldots, m\right.$

- 'exemplos grandes' $\Rightarrow\left\{\begin{array}{l}l_{i} \in[0.10 \mathrm{~L}, 0.50 \mathrm{~L}] \\ w_{i} \in[0.10 \mathrm{~W}, 0.50 \mathrm{~W}]\end{array}, i=1, \ldots, m\right.$

Para avaliarmos a eficiência computacional do método, foram gerados uma amostra de 50 exemplos para cada diferente valor de $m$. Este processo foi repetido para cada uma das classes acima e a análise foi realizada separadamente para cada classe.

Demanda: As coordenadas do vetor de demanda foram determinadas de forma a estarem diretamente relacionadas ao tamanho da peça em estoque e dos itens a serem produzidos, de modo que muitas placas em estoque necessitam ser cortadas: 


$$
d_{i}=\left\lfloor\frac{L * W}{l_{i} * w_{i}}\right\rfloor * 30, \quad i=1, \ldots, m
$$

- O número máximo de iterações para o Método Simplex consideramos sendo igual a $30 * m$.

Feitas as considerações anteriores apresentamos agora os resultados computacionais obtidos com as simulações.

Os resultados estão apresentados primeiramente pelas tabelas 6.1 e 6.2. A figura 6.1 ilustra o comportamento do método simplex.

Tabela 6.1 - Exemplos Pequenos

\begin{tabular}{|c|c|c|}
\hline$m$ & $\alpha$ & $f(m)$ \\
\hline 5 & 0,812 & 4,06 \\
\hline 10 & 1,122 & 11,22 \\
\hline 20 & 2,164 & 43,28 \\
\hline 40 & 5,094 & 203,76 \\
\hline
\end{tabular}

Tabela 6.2 - Exemplos Grandes

\begin{tabular}{|c|c|c|}
\hline$m$ & $\alpha$ & $f(m)$ \\
\hline 5 & 1,28 & 6,4 \\
\hline 10 & 2,112 & 21,12 \\
\hline 20 & 3,976 & 79,52 \\
\hline 40 & 8,006 & 320,24 \\
\hline
\end{tabular}

Onde $f(\mathrm{~m})$ é o número médio de iterações e $\alpha$ é tal que $\alpha=\mathrm{f}(m) / m$.

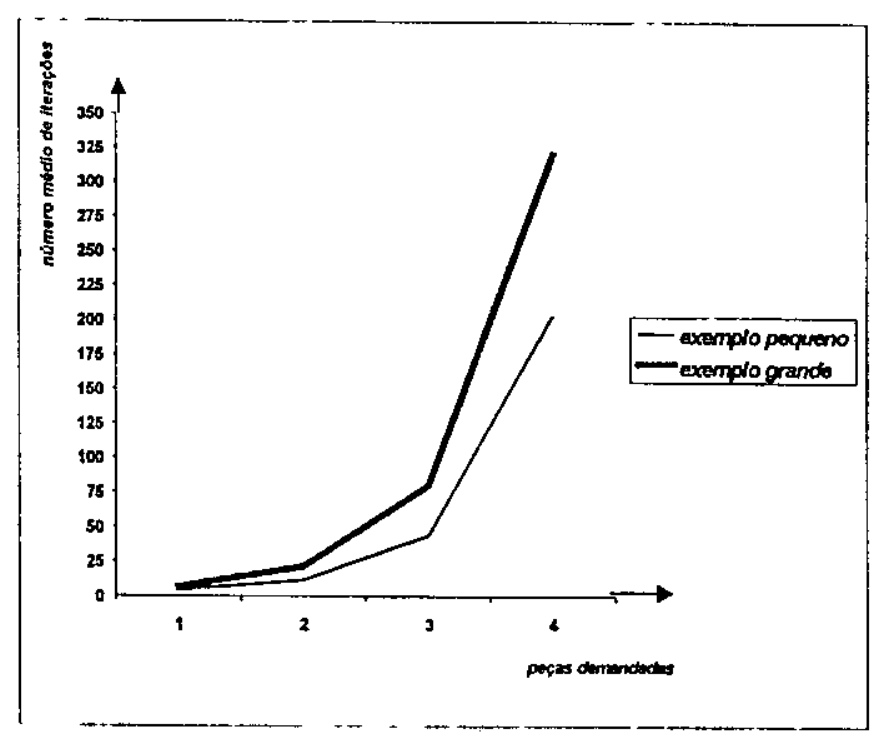

Figura 6.1 - Desempenho do método simplex

Podemos observar que à medida que dobramos o número de itens $(m)$ o valor de $\alpha$ também dobra . Assim, note que o número médio de iterações foi:

$$
\mathrm{f}(m) \cong \beta m^{2} \text {, onde } \beta=\alpha m .
$$

Portanto $\mathrm{f}(m)$ se comporta como função quadrática de $m$. O desempenho do método simplex tem um comportamento polinomial de grau baixo, o que está de acordo com o folclore simplex. 
Uma vez mais o método simplex, apesar de um comportamento ruim do ponto de vista teórico (Klee e Minty, 1972), tem um bom desempenho para classe de problemas de corte de estoque (onde se pode ter milhões de variáveis). Deve-se observar, entretanto, que o tempo de resolução (que depende da geração de colunas,i.e., resolução de vários problemas da mochila) cresce muito mais rapidamente, uma vez que os problemas da mochila são da classe $N P$.

\subsection{Método Dual Simplex}

Para o método dual simplex, os algoritmos implementados foram: dual simplex baseado em penalidades lineares (Arenales 1984), utilizando decomposição LU (Baterls e Golub, 1969) com pivoteamento parcial, e o dual simplex também baseado em penalidades lineares, utilizando a forma produto da inversa. Esses algoritmos foram implementados para problemas lineares com restrições e variáveis canalizadas:

$$
\begin{aligned}
& \text { Minimizar } Z(\mathbf{x})=\mathbf{c}^{\mathbf{T}} \mathbf{x} \\
& \text { Sujeito a: } \mathbf{e} \leq \mathbf{A x} \leq \mathbf{d} \\
& \mathbf{r} \leq \mathrm{x} \leq \mathrm{s}
\end{aligned}
$$

onde $\mathbf{A} \in \mathrm{R}^{m \times n}$.

No entanto os dados a seguir foram obtidos para o algoritmo que utilizou decomposição LU, já que não existiu nenhuma diferença significante no número de iterações com relação ao que utiliza a forma revisada. Porém, em termos de qualidade de solução, o método dual simplex usando decomposição LU obteve melhores soluções comparadas com o da forma revisada, apesar de seu tempo de processamento ser maior em problemas de dimensões maiores, pois o número de operações é maior por iteração.

Para cada tamanho (dimensão) do problema, foram rodados 50 exemplos com os dados gerados aleatoriamente com densidade alta, de tal forma que sempre exista uma solução factível. Os testes foram realizados em um Pentium II $400 \mathrm{MHz}$ com 128 Mbytes de RAM. A linguagem de programação utilizada é a linguagem $\mathrm{C}$ versão 5.01.

Antes de apresentarmos os resultados, considere que as duas primeiras colunas definem os tamanhos dos exemplos. Os tempos e número de iterações são médias dos 50 exemplos.

Nota-se da tabela 6.3, que quando o número de restrições é muito maior do que o número de variáveis (no problema primal), o valor de $\alpha$ é sempre menor que 1, tanto para problemas pequenos quanto para os problemas grandes, o que já era de se esperar. No geral o 
valor de $\alpha$ foi sempre menor do que 2, o que está de acordo com o folclore do método simplex, que preconiza que o número de iterações está entre $2 m$ e $3 m$.

Tabela 6.3

\begin{tabular}{|c|c|c|c|c|}
\hline restriçōes & variáveis & tempo & número médio & \\
\hline$m$ & $n$ & em seg. & de iteraçóes & $\alpha=f(m) / m$ \\
\hline & & & $f(m)$ & \\
\hline 20 & 10 & $0 s$ & 16,22 & 0,81 \\
\hline 40 & 10 & $0 s$ & 18,36 & 0,45 \\
\hline 80 & 10 & $0 s$ & 20,58 & 0,25 \\
\hline & & & & \\
\hline 50 & 50 & $0 s$ & 60,04 & 1,2 \\
\hline 100 & 50 & $0 s$ & 90,38 & 0,9 \\
\hline 200 & 50 & $0 s$ & 115,72 & 0,57 \\
\hline & & & & 1,32 \\
\hline 100 & 100 & $3 s$ & 132,3 & 1,04 \\
\hline 200 & 100 & $4 s$ & 208,12 & 0,79 \\
\hline 300 & 100 & $5 s$ & 238,98 & \\
\hline & & & & 1,48 \\
\hline 200 & 200 & $31 s$ & 296,18 & 1,29 \\
\hline 300 & 200 & $38 s$ & 387,28 & 1,18 \\
\hline 400 & 200 & $60 s$ & 472,24 & \\
\hline
\end{tabular}

Na tabela 6.4 estão os resultados obtidos onde procuramos manter $m$ fixo (para diferentes dimensões) e variar o número de variáveis, para tentar observar, se o aumento no valor de $n$ influencia no número de iterações.

Tabela 6.4

\begin{tabular}{|c|c|c|c|c|}
\hline restriçöes & variáveis & tempo & número médio & \\
\hline$m$ & $n$ & em seg. & de iteraçбes & $\alpha=f(m) / m$ \\
\hline & & & $f(m)$ & \\
\hline 5 & 50 & $0 s$ & 10,25 & 2,05 \\
\hline 5 & 100 & $2 s$ & 15,1 & 3,02 \\
\hline 5 & 400 & $15 s$ & 17,25 & 3,45 \\
\hline & & & & \\
\hline 20 & 50 & $0 s$ & 31,8 & 1,59 \\
\hline 20 & 100 & $5 s$ & 52,5 & 2,6 \\
\hline 20 & 400 & $60 s$ & 63,03 & 3,15 \\
\hline & & & & \\
\hline 100 & 50 & $0 s$ & 87,23 & 0,87 \\
\hline 100 & 200 & $20 s$ & 174,85 & 1,74 \\
\hline 100 & 400 & $240 s$ & 239,1 & 2,39 \\
\hline & & & & \\
\hline 200 & 50 & $0 s$ & 114,12 & 0,57 \\
\hline 200 & 200 & $60 s$ & 304,56 & 1,52 \\
\hline 200 & 400 & $420 s$ & 394,5 & 1,97 \\
\hline & & & & \\
\hline 400 & 50 & $0 s$ & 128,3 & 0,32 \\
\hline 400 & 200 & $60 s$ & 480,78 & 1,2 \\
\hline 400 & 400 & $720 s$ & 696,55 & 1,74 \\
\hline & & & & \\
\hline
\end{tabular}


Pode-se observar, que quando se tem um número bem maior de variáveis $(n \geq 4 \mathrm{~m})$ do que de restrições o valor de $\alpha$ está próximo de 3 , ou seja, o número de variáveis tem uma influência no número de iterações. Veja por exemplo, o caso quando $m=400$ e o número de variáveis passa de 200 para 400 , as iterações aumenta em torno de $80 \%$. Isto é, o número de iterações também depende de $n$, em problemas de médio porte. No entanto, mesmo neste caso confirma o folclore do método simplex.

Quando a razão $m / n \cong 1 / 3$, o número de iterações é normalmente entre $m$ a $2 m$. Mesmo quando essa razão diminui, ou seja, o número de variáveis é bem maior do que o número de restrições, o valor de $\alpha$ parece crescer lentamente, que para os nossos resultados foi em torno de 3. No entanto, existem poucos relatos onde o $n \geq 10 \mathrm{~m}$.

Logo, de um modo geral, os resultados obtidos estão em conformidade com alguns resultados da literatura, como por exemplo Shamir (1987).

\subsection{Método Primal Simplex Revisado (Forma Produto da Inversa)}

O método primal simplex com a regra de Dantzig, foi implementado com a forma produto da inversa para problemas de programação linear da seguinte forma:

$$
\begin{array}{rlrl} 
& \text { Minimizar } & Z(\mathbf{x}) & =\mathbf{c}^{\top} \mathbf{x} \\
\text { Sujeito a: } & \mathbf{A x} & \leq \mathbf{b} \\
& & \mathbf{x} & \geq \mathbf{0}
\end{array}
$$

onde $\mathbf{A}$ tem dimensões $m \times n$.

Como na seção anterior, os dados de entrada foram gerados aleatoriamente, com densidade alta e de modo que exista sempre uma solução factível. Os testes foram executados também em um Pentịum II $400 \mathrm{MHz}$ com 128 Mbytes de RAM.

A matriz base inicial foi a matriz identidade, fornecida pela variáveis de folga, assim tem-se uma solução básica inicial factivel, ou seja, somente a fase II do método simplex foi utilizada. Como o número de iterações requisitadas para a fase I e fase II parecem ser aproximadamente o mesmo (Shamir, 1987), embora haja relatos onde para a fase I seja menor, temos que para análise de desempenho computacional, observar o número de iterações apenas para a fase II é suficiente.

As considerações são as mesmas descritas na seção anterior. Assim temos a tabela 6.5 para problemas pequenos: 
Tabela 6.5

\begin{tabular}{|c|c|c|c|c|}
\hline restrições & Variáveis & tempo & número médio & \\
\hline$m$ & $n$ & em seg. & de iteraçőes & $\alpha=f(m) / m$ \\
\hline & & & $f(m)$ & \\
\hline 5 & 5 & Os & 4,4 & 0,88 \\
\hline 10 & 20 & Os & 10,9 & 1,09 \\
\hline 10 & 40 & 0s & 14,3 & 1,43 \\
\hline 10 & 80 & Os & 18,4 & 1,84 \\
\hline 10 & 200 & Os & 21,8 & 2,18 \\
\hline & & & & \\
\hline 20 & 10 & Os & 8,3 & 0,41 \\
\hline 40 & 10 & 0s & 7,6 & 0,19 \\
\hline 80 & 10 & Os & 7,0 & 0,08 \\
\hline
\end{tabular}

Observa-se da tabela 6.5 , que para esses problemas de pequeno porte, que o número de variáveis pouco influencia no número de iterações. Quando o número de restrições foi maior do que nủmero de variáveis (a razão $\mathrm{m} / \mathrm{n}$ é maior do que dois) o número de iterações diminuiu, possivelmente por que deve ter aumentado o número de restrições redundantes

A tabela 6.6 apresenta os resultados para problemas de dimensões maiores.

Tabela 6.6

\begin{tabular}{|c|c|c|c|c|}
\hline restriçōes & variáveis & tempo & número médio & \\
\hline$m$ & $n$ & em seg. & de iteraçöes & $\alpha=f(m) / m$ \\
\hline 50 & & & $f(m)$ & \\
\hline 50 & 50 & Os & 54,3 & 1,08 \\
\hline 50 & 100 & Os & 87,4 & 1,74 \\
\hline 50 & 300 & $1 s$ & 144,6 & 2,38 \\
\hline 50 & 450 & $1 s$ & 183,3 & 3,69 \\
\hline & & & & \\
\hline 100 & 50 & Os & 55,1 & 0,55 \\
\hline 100 & 100 & 15 & 179,5 & 1,79 \\
\hline 100 & 200 & $1 s$ & 294,3 & 2,94 \\
\hline 100 & 300 & $2 s$ & 452,4 & 4,52 \\
\hline$\cdot$ & & & & 2,55 \\
\hline 200 & 200 & $4 s$ & 510,0 & 9,28 \\
\hline 300 & 300 & $90 s$ & 2785 & \\
\hline
\end{tabular}

Pode-se observar que quando o número de restrições se mantém fixo e o número de variáveis cresce, o número de iterações aumenta na proporção de $n$.

Portanto, dos resultados apresentados anteriormente temos que em geral, o número de variáveis não chega a ter uma influência relevante no desempenho computacional, o qual está em conformidade com os resultados da literatura. No entanto, para os problemas de dimensões $300 \times 300$, nota-se que fator $\alpha$ foi alto, assim como o tempo de processamento. 
Observa-se porém, que situação desse tipo são raras de acontecer, onde o número de iterações é da ordem de $10 m$.

No capítulo VI destacamos alguns procedimentos para melhorar a eficiência do método primal simplex para problemas com dimensões maiores, ou seja em problemas de grande porte.

Temos agora na tabela 6.7 , os resultados da comparação entre o método primal simplex na forma produto da inversa e o método dual simplex revisado baseado em penalidades lineares para problemas do tipo (III).

Tabela 6.7

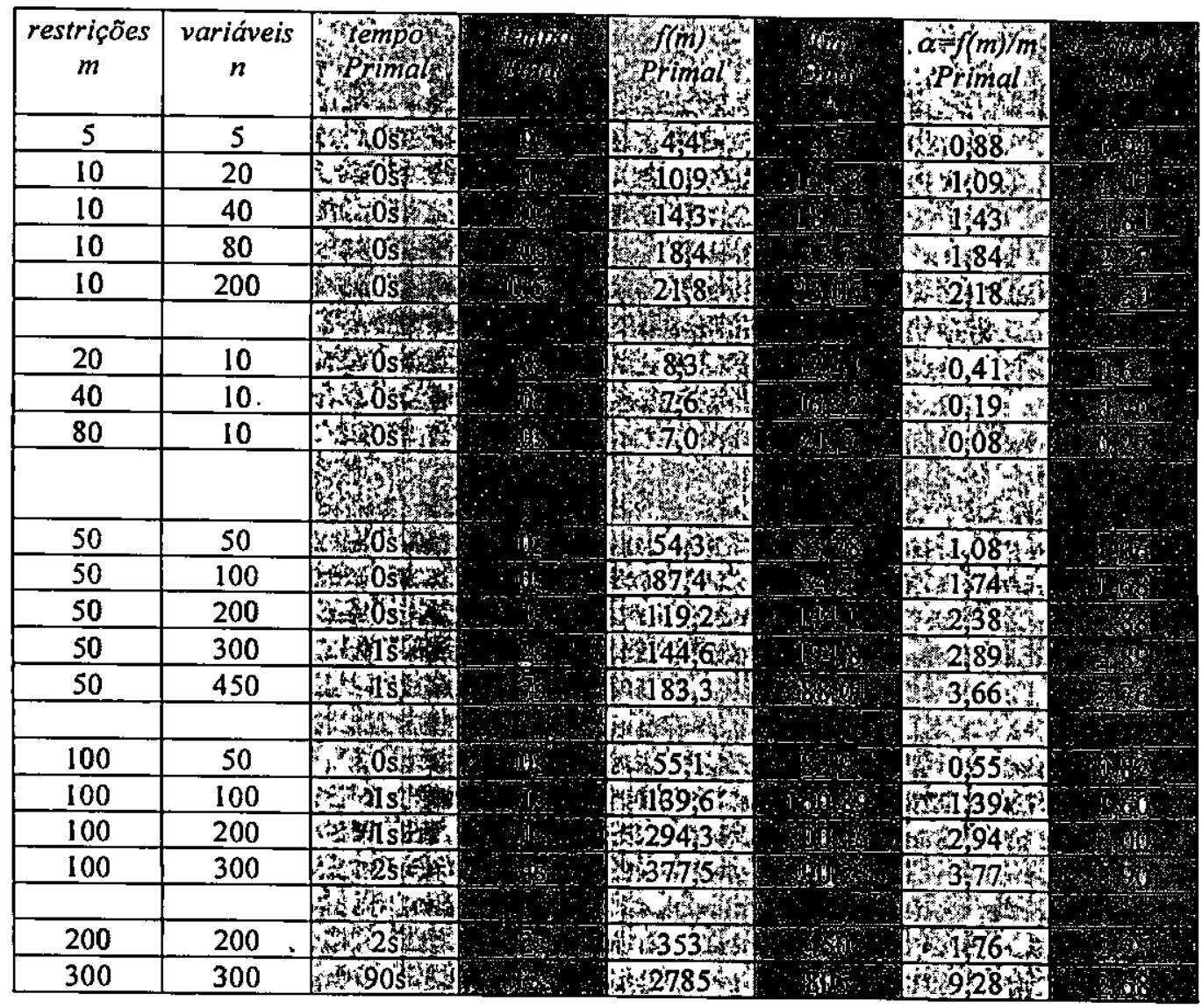

Pode-se observar da tabela 6.7, que de um modo geral o desempenho de método primal simplex é superior com relação ao método dual simplex (Shamir, 1987) para esta classe de problemas (com dimensões inferiores a 300), que são mais fáceis de resolver, seja com relação ao tempo computacional ou com relação ao número de iterações, enquanto que método dual linear por partes tem ótimo desempenho para a classe de problemas canalizados, que são dificeeis de resolver. 


\subsection{Comparações com o Software Cplex 4.0}

Vamos agora comparar os resultados obtidos com método dual simplex linear por partes e com software Cplex 4.0, utilizando o mesmo gerador de exemplo, para a classe de problemas (II).

Para os dados obtidos, o parâmetro do Cplex esteve no valor default, onde ele utiliza um procedimento chamado "Pré-Processador" para simplificar ou reduzir o problema. Porém, não ocorreu nenhuma diferença significante seja no número de iterações ou no tempo de resolução do problema, quando este parâmetro foi mudado. O método utilizado para os resultados do Cplex, foi o método dual simplex (TRANOPT), pois observamos através de alguns testes que o método primal (OPTIMIZE) teve um mau desempenho comparado com o dual.

A tabela 6.8 apresenta os primeiros resultados:

Tabela 6.8

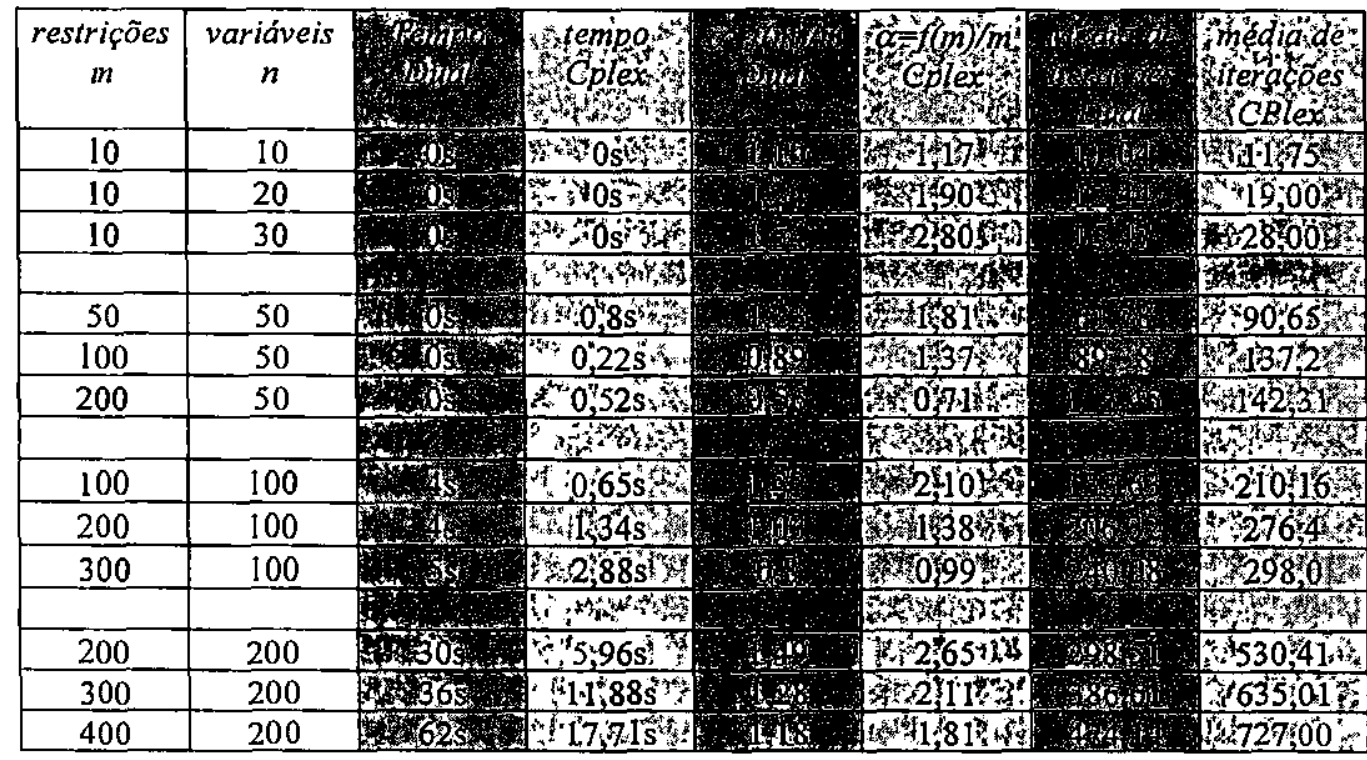

Da tabela 6.8, nota-se que quando o número de variáveis é menor do que 50 e o de restrições menor do que 200 , não tem nenhuma diferença significante no número de iterações e no tempo de processamento, mas quando número de variáveis está fixo em 100, 0 algoritmo implementado começa a ter uma pequena vantagem no número de iterações, porém seu tempo é maior do que o Cplex. Da tabela 6.8, quando se tem $n$ fixo em 200, método dual simplex tem uma desvantagem no tempo computacional, mas o número de iterações é bem menor do que o Cplex. 
Verificando a tabela 6.9, observa-se que quando o número de restrições é 100 ou $200 \mathrm{e}$ o número de variáveis é 200 e 400, a diferença entre o algoritmo implementado e o Cplex, com relação ao número de iterações é grande, onde o algoritmo supera o Cplex, enquanto que este gasta bem menos tempo para a resolução dos problemas. A mesma situação pode ser observada para problemas com onde $m=400$ e $n=200$ ou 400 .

Tabela 6.9

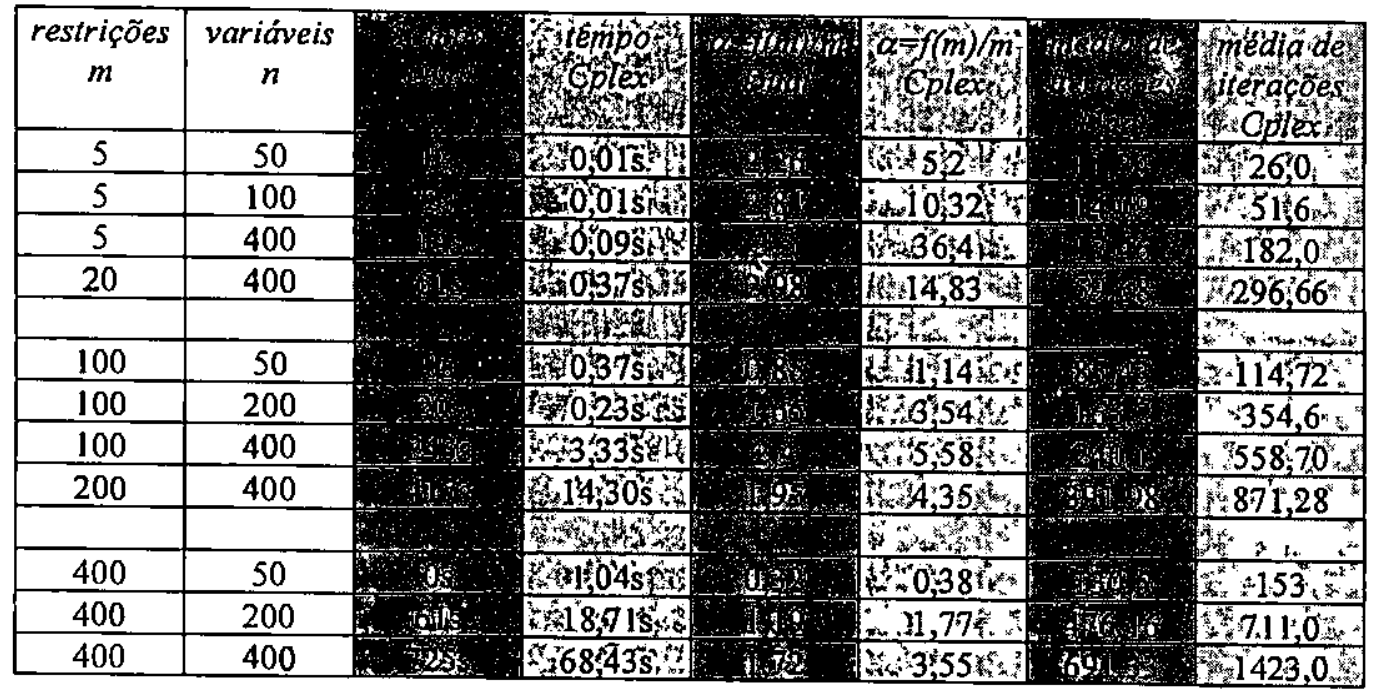

Nota-se que a expectativa era do melhor desempenho do método dual implementado com relação ao Cplex quando o número de restrições é muito maior do que o número de variáveis no problema primal ( $m=400$ e $n=50$ ), já que a base tem dimensão $n \times n$ para o método dual, onde o número de iterações e tempo computacional foi praticamente o mesmo. No entanto para problemas onde $n \gg m(m=20$ e $n=400$, onde a dimensão da base para o algoritmo dual foi $400 \times 400$ ) o método dual teve um ótimo desempenho no número de iterações com relação ao Cplex, porém inferior no tempo de processamento. Isto se deve ao fato do método não explorar a esparsidade na estrutura que ele trabalha.

Assim, de um modo geral, o método dual simplex linear por partes mostrou-se bem superior, do ponto de vista do número de iterações necessárias para resolver o problema com relação ao Cplex, porém teve um tempo alto de tempo de processamento. Portanto, há grandes possibilidades de tornar o algoritmo dual simplex linear por partes mais eficiente em termos do tempo computacional, investindo-se na eficiência da resolução dos sistemas básicos, já que executa um número menor de iterações. 


\section{Capítulo VI}

\section{Conclusões e Propostas Futuras}

Esse trabalho abordou o estudo do clássico método simplex desenvolvido por Dantzig (1947), alguns métodos do tipo simplex como por exemplo o de Anstreicher e Terlaky (1994) e o método dual simplex linear por partes (Arenales, 1984) Revisamos ainda o folclore relacionado com o desempenho computacional do método simplex para a resolução de problemas de programação linear e alguns aspectos relacionados com o mesmo.

Observamos que o método simplex tem um ótimo desempenho computacional em problemas de programação linear obtidos de aplicações práticas ou gerados aleatoriamente (Shamir, 1987). Ou seja, o método simplex tem um comportamento de uma função polinomial em termos do número de restrições de grau baixo e talvez até linear em alguns casos. Isto pode ser visto dos resultados computacionais obtidos com método simplex aplicado ao problema de corte de estoque, que revelam um ótimo desempenho do método primal simplex utilizando a regra de Dantzig, onde este teve comportamento polinomial de grau 2, em problemas que envolvem vários milhares de variáveis, e poucas dezenas de restrições.

Das questões relacionadas à implementação do método simplex para um melhor desempenho do mesmo, citamos o método simplex revisado e a decomposição LU das matrizes básicas, proposta por Bartles e Golub (1969), e notamos que a forma produto da inversa que é baseada na técnica de eliminação de Gauss-Jordan para resolver os sistemas de equações, é eficiente para problemas de pequeno e médio porte, onde o maior deles teve 300 restrições e 300 variáveis. No entanto, de algumas experiências computacionais a técnica mais 
popularmente usada pela maioria dos pacotes de software para programação linear é a fatoração LU, que é baseada na estratégica mais eficiente de triangularização Gaussiana. Este procedimento é muito útil quando o problema é de grande porte e esparso. Além disso, podese recorrer à técnica de pivoteamento parcial, evitando-se a propagação descontrolada dos erros de arredondamento.

Observamos uma diferença não significante, por volta da quarta casa decimal, quando comparamos a qualidade das soluções do algoritmo simplex utilizando as duas técnicas, já que os problemas eram de porte médio e com densidade alta.

Portanto os resultados computacionais que realizamos confirmam o folclore do método simplex em geral e revelam um menor número de iterações do método dual simplex linear por partes (Arenales, 1984) com relação ao Cplex. Porém tal método teve um tempo alto de tempo de processamento, em algumas situações. Portanto, pode-se melhorar o algoritmo para que se torne mais eficiente em termos de tempo computacional, quando o problema apresenta uma característica particular, como por exemplo o número de variáveis for muito grande comparado com o número de restrições para o problema primal.

\section{Propostas Futuras:}

Em muitos problemas reais, o número de variáveis é muito grande comparado com o número de restrições, da ordem de milhares. Portanto, verificar todos os índices não básico das variáveis candidatas para entrar base, é provável que este passo seja muito caro. Porém, não há nenhuma exigência que todos os índices sejam verificados. Então pode-se empregar o seguinte procedimento, chamado custo parcial.

Inicialmente, examina-se somente uma fração do índices não básicos e seleciona-se alguns índices desta fração tendo custo reduzido mais negativo. Vanderbei (1997) sugere verificar $n / 3$ índices não básicos e selecionar os 40 "melhores" entre eles. Então utiliza-se somente parte dessa fração de índices não básicos no algoritmo simplex para as iterações subseqüentes até menor do que uma certa fração (1/2 segundo Vanderbei, 1997)) deles permaneçam aptos a entrar na base. Neste momento, calcula-se os valores atuais de um grupo novo de variáveis não básicas, e retorna-se ao começo deste processo trabalhando com os melhores índices não básicos.

Então, em casos onde $n$ é enorme com relação a $m$, o custo parcial pode ser utilizado fazendo ó uso do método primal simplex. Bazarra (1990) descreve uma implementação particular deste processo usando regra de Dantzig, para selecionaw a variável de entrada, utilizada para problemas de grande porte. 
Da última seção do capítulo III, vemos que o variante simplex com a regra de Dantzig normalizada, com base na experiência computacional de Goldfarb e Reid (1992), pode ser utilizado para o método primal simplex assim como para o dual, para resolver problemas dificeis de programação linear de grande porte.

E ainda, pode-se trabalhar com implementações do método primal simplex utilizando o procedimento de custo parcial, regra de Dantzig normalizada e que explore esparsidade quando esta se faz presente. Para o método dual simplex linear por partes pode-se melhorar o algoritmo, explorando a esparsidade dos dados de entrada, para que este se torne mais eficiente em termos de tempo computacional.

Para os principais algoritmos do tipo simplex, tem sido possível construir "exemplos dificeis", que exigem um número exponencial de iterações. Seria importante identificar classes de problemas dificeis para métodos do tipo simplex e trabalhar no sentido da questão mais desafiante da teoria de otimização linear, sobre a existência ou não de algoritmo do tipo simplex com complexidade polinomial. 


\section{Referências Bibliográficas}

Adler et al. (1986) "An Implementation of Karmarkar's Algorithm for Linear Programming" Report ORC86-8, Operations Research, University of California, Berkeley.

Anstreicher, K. M. and Terlaky, T. (1990) "A Monotonic Build-up Simplex Algorithm" Report 91-82. Faculty of Techincal Mathematics and Informatics, Delft University of Technology, The Netherlands.

Anstreicher, K. M. and Terlaky, T. (1994) "A Monotonic Build-up Simplex Algorithm for Linear Programming" Ops. Research 42, 556-561.

Anstreicher, K. M. and Terlaky, T. (1996) "Potential Reduction Methods, in Interior Point Methods in Mathematical Programming" Kluwer Academic Publishers.

Arenales, M. N. (1984) "Programação Linear: Novos Métodos e Alguns Problemas Particulares" Tese de Doutorado, Unicamp/FEC.

Arenales, M. e Morábito, R. (editores) (1997) "Mini Curso: O Problema de Corte e Empacotamento e Aplicações Industriais". In: XX CNMAC - Congresso Nacional de Matemática Aplicada e Computacional, Gramado - R.S., 1-23. 
Avis, D and Chavátal, V. (1978) "Notes on Bland's Pivoting Rule" Math. Programming Study 8, 24-34.

Balbo, A. R. (1991) "Método de Pontos Interiores com Mudança de Escala para Programação Linear" Dissertação de Mestrado, ICMC-USP.

Barnette, D. W. (1971) "The Minimum Number of Vertices of a Simple Polytope" Israel J. Math. 10, vol 1, 121-125.

Bazaraa, M. S. et al. (1990) "Linear Programming and Network Flows" John Wiley \& Sons.

Benichou, M., et al. (1977) "The Efficient solution of Large-Scale Linear Programming Problems - Some Algorithmic Techiniques and Computational Results" Math. Programming 13, 281-322.

Bland, R. G. (1977) "New Finite Pivoting Rules For the Simplex Method" Math. Oper. Res., 2, vol 2, 103-107.

Bland, R. G. (1977) "A Combinatorial Abstraction of Linear" J. Combin. Theory (Ser. B) 23, 33-57.

Chen H. et al. (1994) "The Simplex Algorithm with a New Priaml and Dual Pivot Rule" Operations Research Letters 16, 121-127.

Crowder, H. and Hatting, J. M. (1975) "Partially Normalized Pivot Selection in Linear Programming" Math. Programming Study 4, 12-25.

Cunningham, W. H. (19 76) “A Network Simplex Method” Math. Programming 11, 105-116.

Dantizg, G. B. (1963) "Linear Programming and Extensions". Princeton University Press. 
Dantizg, G. B. (1979) "Comments on Khachian's Algorithm for linear Programming" Technical Report SOL 79-22, Department of Operations Research, Stanford University.

Dantizg, G. B and Thapa, M. N. (1997) “Linear Programming” Ed. Springer Verlag.

Dickson, J. C. and Frederick, F. P. (1960) "A Decision Rule for Improvement Efficiency in Solving Linear Programming Problems with the Simplex Algorithm" Comm. ACM 3, 509-512.

Dunham, et al. (1977) "Some Experimental Results Concerning the Expected Number of Pivots for Solving Randomly Generated Linear Programs" TR 77-16, Operations Research and System Analysis Department, University of North Carolina at Chapel Hill.

Folkman, J. and Lawrence, J. (1978) "Oriented Matroids" J. Combin. Theory (Ser. B) $25,199-236$.

Freund, R. M. and Mizuno, S. (1996) "Interior Point Methods: Current Status and Future Directions"

Fukuda, K. (1982) “Oriented Matroid Programming" Ph.D. Thesis, Waterloo University, Waterloo, Canada.

Garey, M. R. and Johnson, D. S. (1979) "Computers and Intractability: A Guide to the Theory of NP-Completeness" W. H. Freeman \& Company, San Francisco, CA.

Goldfarb, D. and Forrest, J. J. H. (1992) "Steepest Edge Simplex Algorithm for Linear Programming" Math. Programming 57, 341-374.

Goldfarb, D. and Reid, J. K. (1977) “A Practicable Steepest-Edge Simplex Algorithm" Math. Programming 12, 361-371.

Goldfarb, D. and Sit, W. Y. (1979) "Worst Case Behavior of the Steepest Edge Simplex Method" Discrete Appl. Math., 277-285. 
Gonzaga, C. (1992) "Path Following Methods for Linear Programming" SIAM Review 34, vol 2, 167-227.

Haimovich, M., (1983) "The Simplex Algorithm is Very Good! - On the Expected Number of Pivot Steps and Related Properties of Random Linear Programs" Draft, 415 Uris Hall, Columbia University, New York.

Hertog, D. (1994) "Interior Point Approach to Linear, Quadratic and Convex Programming, Algorithms, and Complexity" Kluwer Publishers, Dordrecht, The Netherlands.

Ho, J. K. and Loute, E. (1980) "A Comparative Study of Two Methods of Staircase Linear Programs". ACM Trans. Math. Software, 6, vol 1, 17-30.

Ho, J. K. and Loute, E. (1983) "Computational Experience with Advanced Implementation of Decomposition Algorithms for Linear Programming". Math. Programming 27, 283-290.

Hung, M. S. (1983) "A Polynomial Simplex Method for the Assignment Problem" Operations Research 31, vol 3, 595-600.

Jansen, B et al. (1995) "A Short Survey on Ten Years of Interior Point Methods" Report 95-45, Delft University of Techbology, Delf, The Netherlands.

Jeroslow, R. G. (1973) “The Simplex Algorithm with the Pivot Rule Of Maximizing Improvement Criterions". Discrete Mathematics 4, 367-378.

Kamarkar, N. (1984) "A New Polynomial-Time Algorithm for Linear Programming" . Combinatorica 4, 373-395.

Khachian, L. G. (1979) "A Polynomial Algorithm for Linear Programming" Dokla. Akad. Nauk. USSR, 244, vol 5, 1093-96.

Klee, V. (1964) “Diameters of Polyedral Graphs" Canad. J. Math. 16, 602-614. 
Klee, V. (1974) "Polytope Pairs and their Relationship to Linear Programming" Acta Math. 133, 11-25.

Klee, V. and Minty, G. J. (1972) "How Good is the Simplex Algorithm?" in Shish, Proc. 3rd Sympos. Inequalities, AP, New York, 159-175.

Klee, V. and Walkup, D. (1967) "The d-Step Comjecture for Polyhedra of Dimension $d<6$ " Acta Math. 117, 53-87.

Kuhn, H..W. and Quandt, R. E. (1963) "An Experimental Study of the Simplex Method" in Metropolis et al. (Eds.), Proc. 15th Sympos. Appl. Math., Amer. Math. Soc., Providence, R.I., 107-124.

Larman, D. G. (1970) "Paths on Polytopes" Proc. London Math. Soc. 3, vol 20, $161-178$.

Lawler, E. L. (1970) "The Great Mathematical Sputnik of 1979" The Science.

Lawrence, J. (1975) "Oriented Matroids" Dissertation, University of Washington.

Lovász, L. (1980) "A New Linear Programming Algorithm - Better or Worse Than The Simplex Method?" Math. Intelligencer 2, vol 3, 141-146.

Lustig, I. (1989) "An Analysis of na Available Set of Linear Programming Test Problems" Comput. Opns. Res. 16,vol 2, 173-184.

Lustig, I. et al. (1994) "Interior Point Methods: Computational State of the Art" ORSA Journal on Computing 6, 1-14.

Marsten, R. E. (1974) "An Algorithm for Large Set Partitioning Problems" Management Sci.20, vol 5, 774-787.

Murty, K. G. (1983) "Linear Programming” John Wiley \& Sons. 
Orlin, J. B. (1983) “A Polynomial - Time Paranetirc Simplex Algorithm for the Minimum Cost Network Flow Problem" Working Paper, Sloan School of Management, MIT.

Pinto, M. J. (1999) "O Problema de Corte de Estoque Inteirol" Dissertação de Mestrado, ICMC-USP.

Roos, C. (1990) “An Exponential Example for Terlaky's Pivoting Rule for the Criss-Cross Simplex Method'Math. Progr. 46, 78-94.

Sethi, A P. and Thompson, G. L. (1983) "The Non-candiddate Constraint Method for Reducing the size of a Linear Program" Redundancy in Mathematical Programming.

Sethi, A P. and Thompson, G. L. (1984) "The Pivot and Probe Algorithm for Solving a Linear Program" Mathematical Programming 29, 219-233.

Shamir, R. (1987) "The Efficiency of the Simplex Method: A Survey" Management Science 3, 301-334.

Terlaky, T. (1987) "A Finite Criss-Cross Method for Oriented Matroids" J. Combin. Theory (Ser. B) 42, 319-327.

Terlaky, T. and Zhang, S. (1993) "Pivot Rules for Linear Programming: A Survey on Recent Theorical Developments" Annals of Operation Research 46, 203-233.

Todd, M. J. (1980) "The Monotonic Bounded Hirsch Conjecture is False for Dimension at least 4" Math Oper. Res. 5, vol 4, 599-601.

Todd, M. J. (to appear) "Potential Reduction Methods in Mathematical Programming" Mathematical Programming B.

Traub, J. F. and Wozniakowski, H. (1982) "Complexily of Linear Programming" Oper. Res. Lett. 1, vol 2, 59-62.

Vanderbei, R. J. (1997) "Linear Programming: Foundations and Extensions" Kluwer Academic Publishers. 
Wolfe, P. (1980) "The Ellipsoid Algorithm" (Letter to the editor), Science 208, 240-242.

Wolfe, P. and Cutler, L. (1963) "Experiments in Linear Programming" in Graves and Wolfe (Eds.) Recent Advances in Mathematical Programming, McGraw Hill, New York, 177 200.

Zhang, S. (1999) "New Variants of Finite Criss-Cross Pivot Algorithms for Linear Programming" European Journal of Operational Research 116, 607-614.

Zionts, S. (1969) “The Criss-Cross Method for Solving Linear Programming Problems" Manag. Sci. 15, 426-445. 


\section{Apêndice}

\section{O Quadro Simplex}

Apresentaremos agora brevemente, o método simplex no chamado "formato tableau". Suponha que uma solução básica primal factível $\mathbf{x}$ esteja disponível com a base B. Assim o problema (1.1) apresentado no capítulo I, pode ser representado como:

$$
\begin{aligned}
& \text { Minimizar } Z(x)=c_{B}^{T} x_{B}+c_{N}^{T} x_{N}
\end{aligned}
$$

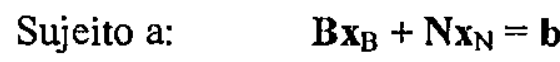

$$
\begin{aligned}
& \mathbf{x}_{\mathrm{B}}, \quad \mathbf{x}_{\mathrm{N}} \geq 0
\end{aligned}
$$

ou equivalentemente

$$
\begin{aligned}
& \text { Minimizar } Z(\mathbf{x})-\mathbf{c}_{\mathrm{B}}^{\mathrm{T}} \mathbf{B}^{-1} \mathbf{b}=\mathbf{O} \mathbf{x}_{\mathrm{B}}+\overline{\mathbf{c}}_{\mathrm{N}} \mathbf{x}_{\mathrm{N}} \\
& \text { Sujeito a: } \quad \mathbf{x}_{\mathrm{B}}+\mathbf{y} \mathbf{x}_{\mathrm{N}}=\overrightarrow{\mathbf{b}} \\
& \mathbf{x}_{\mathrm{B}}, \quad \mathbf{x}_{\mathrm{N}} \geq 0
\end{aligned}
$$

onde $\quad \overline{\mathbf{c}}_{\mathrm{N}}=\mathbf{c}_{\mathrm{N}}-\pi^{\mathrm{T}} \mathrm{N},\left(\pi=\mathbf{c}_{\mathrm{B}}^{\mathrm{T}} \mathrm{B}^{-1}\right)$

$$
\begin{aligned}
& \overline{\mathbf{b}}=\mathbf{B}^{-1} \mathbf{b} \\
& \mathbf{y}=\mathbf{B}^{-1} \mathbf{N} .
\end{aligned}
$$

Mais detalhadamente, podemos expor todas as informações relativas à base $\mathbf{B}$ no quadro abaixo: 


\begin{tabular}{|c|c|c|c|c|c|c|c|c|c|c|}
\hline & $\cdots x_{j}$ & $\cdots$ & $x_{k}$ & $\cdots$ & $x_{1}$ & $\cdots$ & $x_{i}$ & $\cdots$ & & \\
\hline$-Z(\mathbf{x})$ & $\cdots \bar{c}_{j}$ & $\cdots$ & $\bar{c}_{k}$ & $\cdots$ & 0 & $\ldots$ & 0 & $\cdots$ & 0 & $-\mathbf{c}_{\mathrm{B}}^{\mathrm{T}} \overline{\mathbf{b}}$ \\
\hline $\begin{array}{c}\mathrm{X}_{\mathrm{B}_{1}} \\
\vdots\end{array}$ & $\begin{array}{c}\cdots y_{1, j} \\
\vdots\end{array}$ & $\begin{array}{l}\cdots \\
\ddots\end{array}$ & $\begin{array}{c}y_{1 \mathrm{k}} \\
\vdots\end{array}$ & $\cdots$ & $\begin{array}{l}1 \\
\vdots\end{array}$ & 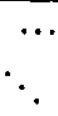 & 0 & $\cdots$ & 0 & $\begin{array}{c}\overrightarrow{\mathrm{b}}_{\mathrm{I}} \\
\vdots\end{array}$ \\
\hline $\begin{array}{c}\mathrm{X}_{\mathrm{B}_{\mathrm{r}}} \\
\vdots\end{array}$ & $\begin{array}{c}\cdots y_{r, j} \\
\vdots\end{array}$ & $\cdots$ & $\begin{array}{c}y_{\text {rk }} \\
\vdots\end{array}$ & $\begin{array}{l}\cdots \\
\ddots\end{array}$ & $\begin{array}{c}0 \\
\vdots\end{array}$ & $\cdots$ & 1 & $\cdots$ & 0 & $\begin{array}{c}\overline{\mathrm{b}}_{\mathrm{r}} \\
\vdots\end{array}$ \\
\hline$x_{B_{m}}$ & $\cdots y_{m, j}$ & $\cdots$ & $y_{m k}$ & $\cdots$ & 0 & $\cdots$ & 0 & $\cdots$ & 1 & $\overline{\mathrm{b}}_{\mathrm{m}}$ \\
\hline
\end{tabular}

A primeira linha contém todas os custos relativos e o valor da função objetivo; nas linhas restantes aprecem as coordenadas das direções simplex (com o sinal oposto): $\mathbf{B}^{-1} \mathbf{a}_{\mathrm{k}} \mathbf{e}$, na última coluna o valor das variáveis básicas: $\bar{b}=\mathbf{B}^{\cdot 1} \mathbf{b}$. 